\title{
STATISTICAL REGULARITIES OF SELF-INTERSECTION COUNTS FOR GEODESICS ON NEGATIVELY CURVED SURFACES
}

\author{
STEVEN P. LALLEY
}

\begin{abstract}
Let $\Upsilon$ be a compact, negatively curved surface. From the (finite) set of all closed geodesics on $\Upsilon$ of length at most L, choose one, say, $\gamma_{L}$, at random, and let $N\left(\gamma_{L}\right)$ be the number of its self-intersections. It is known that there is a positive constant $\kappa$ depending on the metric such that $N\left(\gamma_{L}\right) / L^{2} \rightarrow \kappa$ in probability as $L \rightarrow \infty$. The main results of this article concern the size of typical fluctuations of $N\left(\gamma_{L}\right)$ about $\kappa L^{2}$. It is proved that if the metric has constant curvature -1 , then typical fluctuations are of order $L$; in particular, as $L \rightarrow \infty$ the random variables $\left(N\left(\gamma_{L}\right)-\kappa L^{2}\right) / L$ converge in distribution. In contrast, it is also proved that if the metric has variable negative curvature, then fluctuations of $N\left(\gamma_{L}\right)$ are of order $L^{3 / 2}$; in particular, the random variables $\left(N\left(\gamma_{L}\right)-\kappa L^{2}\right) / L^{3 / 2}$ converge in distribution to a Gaussian distribution with positive variance. Similar results are proved for generic geodesics, that is, geodesics whose initial tangent vectors are chosen randomly according to normalized Liouville measure.
\end{abstract}

\section{Contents}

1. Introduction . . . . . . . . . . . . . . . . . . . . 1192

2. Intersection kernel . . . . . . . . . . . . . . . . . . . . . . 1196

3. Symbolic dynamics . . . . . . . . . . . . . . . . . . . . . . . . . . 1204

4. Gibbs states and thermodynamic formalism . . . . . . . . . . . . . . . 1214

5. $U$-statistics . . . . . . . . . . . . . . . . . . . . . . . . . . . 1219

6. Cohomology and Hoeffding projections in constant and variable negative curvature . . . . . . . . . . . . . . . . . . . . . . 1228

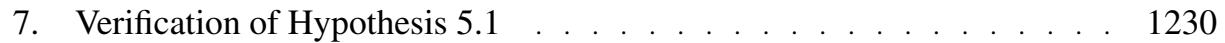

8. Proof of Theorem 1.1 . . . . . . . . . . . . . . . . . . . . . 1238

9. Proof of Theorem 1.2 . . . . . . . . . . . . . . . . . . . . . . . . . . . . . . 1240

DUKE MATHEMATICAL JOURNAL

Vol. 163, No. 6, () 2014 DOI 10.1215/00127094-2649425

Received 15 November 2011. Revision received 13 September 2013.

2010 Mathematics Subject Classification. Primary 57M05; Secondary 53C22, 37D40.

Author's work partially supported by National Science Foundation grant DMS-1106669. 
10. $U$-statistics and randomly chosen periodic orbits . . . . . . . . . . . 1245

11. Proof of Theorem 1.3 . . . . . . . . . . . . . . . . . . . 1256

References . . . . . . . . . . . . . . . . . . 1258

\section{Introduction}

\subsection{Self-intersections of random geodesics}

Choose a point $x$ and a direction $\theta$ at random on a compact, negatively curved surface $\Upsilon$-that is, so that the distribution of the random unit vector $(x, \theta)$ is the normalized Liouville measure on the unit tangent bundle $S \Upsilon$-and let $\gamma(t)=\gamma(t ; x, \theta)$ be the unit speed geodesic ray in direction $\theta$ started at $x$, viewed as a curve in $S \Upsilon$. Let $p: S \Upsilon \rightarrow \Upsilon$ be the natural projection, and denote by $N(t)=N(\gamma[0, t])$ the number of transversal ${ }^{\dagger}$ self-intersections of the geodesic segment $p \circ \gamma[0, t]$. For large $t$, the number $N(t)$ will be of order $t^{2}$; in fact,

$$
\lim _{t \rightarrow \infty} N(t) / t^{2}=1 /(4 \pi|\Upsilon|):=\kappa \Upsilon
$$

with probability 1 . See Section 2.3 below for the (easy) proof. A similar result holds for a randomly chosen closed geodesic (see [23, Theorem 1]): if, from among all closed geodesics of length at most $L$, one is chosen at random, then the number of self-intersections normalized by $L^{2}$ will, with probability approaching 1 as $L \rightarrow \infty$, be close to $\kappa_{\Upsilon}$ (see [33, Theorem 1] for a related theorem). Closed geodesics with no self-intersections have long been of interest in geometry—see, for instance, [5], [6], [27] — and it is known (see [27], [36]) that the number of simple closed geodesics of length at most $t$ grows at a polynomial rate in $t$. The fact that there are arbitrarily long simple closed geodesics implies that the maximal variation in $N(t)$ is of order $t^{2}$. The problems we address in this paper concern the order of magnitude of typical variations of the self-intersection count $N(t)$ about $\kappa_{\Upsilon} t^{2}$ for both random and random closed geodesics. For random geodesics, the main result is the following theorem.

\section{THEOREM 1.1}

Let $\Upsilon$ be a compact surface equipped with a Riemannian metric of negative curvature. Assume that $u=(x, \theta)$ is a random unit tangent vector distributed according to normalized Liouville measure on $S \Upsilon$, and let $N(T)$ be the number of transversal self-intersections of the geodesic segment $\gamma([0, T] ; x, \theta)$ with initial tangent vector $u$. Then as $T \rightarrow \infty$,

\footnotetext{
${ }^{\dagger}$ If the initial point $x$ and direction $\theta$ are chosen randomly (according to the normalized Liouville measure on the unit tangent bundle), then there is probability 0 that the resulting geodesic will be periodic, so with probability 1 every self-intersection will necessarily be transversal.
} 


$$
\frac{N(T)-\kappa \Upsilon T^{2}}{T} \stackrel{D}{\longrightarrow} \Psi
$$

for some probability distribution $\Psi$ on $\mathbb{R}$ (which will in general depend on the surface and the Riemannian metric).

Here $\stackrel{\mathcal{D}}{\rightarrow}$ indicates convergence in distribution (i.e., weak convergence; see [4]): a family of real-valued random variables $Y_{t}$ is said to converge in distribution to a Borel probability measure $G$ on $\mathbb{R}$ if, for every bounded, continuous function $f$,

$$
\lim _{t \rightarrow \infty} E f\left(Y_{t}\right)=\int f d G
$$

Since to first order $N(T)$ is approximately $\kappa \Upsilon T^{2}$, and since $T=\sqrt{T^{2}}$, Theorem 1.1 might at first sight appear to be typical "central limit" behavior (see [34] for the classical central limit theorem for geodesic flows). But it is not. The limit distribution $\Psi$ in (2) is a limit of Gaussian quadratic forms, and therefore is most likely not Gaussian. ${ }^{\dagger}$ Moreover, a closer look will show that for central limit behavior the typical order of magnitude of fluctuations should be $T^{3 / 2}$, not $T$. This is what occurs for localized self-intersection counts, as we now explain.

Label the points of self-intersection of $\gamma([0, T])$ on $\Upsilon$ as $x_{1}, x_{2}, \ldots, x_{N(T)}$ (the ordering is irrelevant). For any smooth, nonnegative function $\varphi: \Upsilon \rightarrow \mathbb{R}_{+}$, define the $\varphi$-localized self-intersection count $N_{\varphi}(T)$ by

$$
N_{\varphi}(T)=N_{\varphi}(\gamma[0, T])=\sum_{i=1}^{N(T)} \varphi\left(x_{i}\right) .
$$

Like the global self-intersection count $N(T)$, the localized self-intersection count $N_{\varphi}(T)$ grows quadratically in $T$; in particular, if the initial tangent vector $(x, \theta)$ is chosen randomly according to the normalized Liouville measure, then with probability 1 ,

$$
\lim _{T \rightarrow \infty} \frac{N_{\varphi}(T)}{T^{2}}=\kappa \Upsilon\|\varphi\|_{1}
$$

where $\|\varphi\|_{1}$ denotes the integral of $\varphi$ against the normalized surface area measure on $\Upsilon$.

\footnotetext{
${ }^{\dagger}$ A Gaussian quadratic form is a random variable of the form $\sum_{i=1}^{m} \sigma_{i}^{2} Z_{i}^{2}$, where the random variables $Z_{i}$ are independent standard Gaussians. The proof of Theorem 1.1 will show that $\Psi$ is the weak limit of some sequence of such random variables. Unfortunately, it does not seem possible to compute the variances $\sigma_{i}^{2}$ in these approximating quadratic forms, so the limit distribution $\Psi$ cannot be explicitly identified.
} 


\section{THEOREM 1.2}

For any compact, negatively curved surface $\Upsilon$ there is a constant $\varepsilon>0$ with the following property. If $\varphi \geq 0$ is smooth and not identically zero but has support of diameter less than $\varepsilon$, then under the hypotheses of Theorem 1.1, for some constant $\sigma>0$ depending on $\varphi$,

$$
\frac{N_{\varphi}(T)-\kappa \Upsilon\|\varphi\|_{1} T^{2}}{\sigma T^{3 / 2}} \stackrel{D}{\longrightarrow} \Phi
$$

as $T \rightarrow \infty$. Here $\Phi$ is the standard unit Gaussian distribution on $\mathbb{R}$.

In the course of the proof, we will show that a lower bound for $\varepsilon$ is the distance between two nonintersecting closed geodesics.

\subsection{Self-intersections of closed geodesics}

On any compact, negatively curved surface there are countably many closed geodesics, and only finitely many with length in any given bounded interval $[0, L]$. According to the celebrated prime geodesic theorem of Margulis [25], [26, Section 6, Theorem 5], the number $\pi(L)$ of closed geodesics of length at most $L$ satisfies the asymptotic law

$$
\pi(L) \sim \frac{e^{h L}}{h L} \quad \text { as } L \rightarrow \infty,
$$

where $h>0$ is the topological entropy of the geodesic flow. Furthermore, the closed geodesics are equidistributed according to the maximum entropy measure, in the following sense (see [22]): if a closed geodesic is chosen at random from among those of length at most $L$, then with probability approaching 1 as $L \rightarrow \infty$, the empirical distribution of the geodesic chosen will be in a weak neighborhood of the maximum entropy measure. (See also Bowen [9] for a somewhat weaker statement. It does not matter whether the random closed geodesic is chosen from the set of prime closed geodesics or the set of all closed geodesics, because Margulis's theorem implies that the number of nonprime closed geodesics of length at most $L$ is $O\left(e^{h L / 2}\right)$.) In addition, the maximum entropy measure governs the statistics of closed geodesics even at the level of "fluctuations," in that central limit theorems analogous to those governing random geodesics (see [34]) hold for randomly chosen closed geodesics (see [20] and [21] for precise statements). Thus, it is natural to expect that the maximum entropy measure also controls the statistics of self-intersections.

For compact surfaces of constant negative curvature the maximum entropy measure and the (normalized) Liouville measure coincide, so it is natural to expect that in this case there should be some connection between the fluctuations in self-intersection counts of closed geodesics with those of random geodesics. For compact surfaces of variable negative curvature, however, the maximum entropy measure and the Liou- 
ville measure are mutually singular. Thus, it is natural to conjecture that the fluctuations of self-intersection counts in the constant curvature and variable curvature cases obey different statistical laws. The next theorem asserts that this is the case.

THEOREM 1.3

Let $\Upsilon$ be a compact surface of negative curvature, let $\gamma_{L}$ be a closed geodesic randomly chosen from the $\pi(L)$ closed geodesics of length at most $L$, and let $N\left(\gamma_{L}\right)$ be the number of self-intersections of $\gamma_{L}$.

(A) If $\Upsilon$ has constant negative curvature, then for some probability distribution $\Psi$ on $\mathbb{R}$, as $L \rightarrow \infty$,

$$
\frac{N\left(\gamma_{L}\right)-\kappa_{\Upsilon} L^{2}}{L} \stackrel{D}{\longrightarrow} \Psi .
$$

(B) If $\Upsilon$ has variable negative curvature, then for some constants $\sigma>0$ and $\kappa^{*}>0$,

$$
\frac{N\left(\gamma_{L}\right)-\kappa^{*} L^{2}}{L^{3 / 2}} \stackrel{D}{\longrightarrow} \Phi
$$

where $\Phi$ is the standard unit Gaussian distribution.

In the variable curvature case, the constant $\kappa^{*}$ need not in general be the same as the constant $\kappa \Upsilon$ in the corresponding law (2), because the self-intersection statistics for closed geodesics are governed by the maximum entropy-invariant measure, whereas for random geodesics they are governed by the Liouville measure.

Similar results hold for the number of intersections of two randomly chosen closed geodesics. Let $\gamma_{L}$ and $\gamma_{L}^{\prime}$ be independently chosen at random from the set of closed geodesics of length at most $L$, and let $N\left(\gamma_{L}, \gamma_{L}^{\prime}\right)$ be the number of intersections of $\gamma_{L}$ with $\gamma_{L}^{\prime}$. (If by chance $\gamma_{L}=\gamma_{L}^{\prime}$, set $N\left(\gamma_{L}, \gamma_{L}^{\prime}\right)=N\left(\gamma_{L}\right)$. Because the probability of choosing the same closed geodesic twice is $1 / \pi(L) \rightarrow 0$, this event has negligible effect on the distribution of $N\left(\gamma_{L}, \gamma_{L}^{\prime}\right)$ in the large $L$ limit.)

THEOREM 1.4

If $\Upsilon$ has constant negative curvature, then for some probability distribution $\Psi^{*}=\Psi_{\Upsilon}^{*}$ on $\mathbb{R}$,

$$
\frac{N\left(\gamma_{L}, \gamma_{L}^{\prime}\right)-\kappa \Upsilon L^{2}}{L} \stackrel{D}{\longrightarrow} \Psi^{*}
$$

as $L \rightarrow \infty$. If $\Upsilon$ has variable negative curvature, then

$$
\frac{N\left(\gamma_{L}, \gamma_{L}^{\prime}\right)-\kappa^{*} L^{2}}{L^{3 / 2}} \stackrel{D}{\longrightarrow} \Phi
$$

We omit the proof, as it is very similar to that of Theorem 1.3. 
It is noteworthy that in constant negative curvature the order of magnitude of typical fluctuations in Theorems 1.3 and 1.4 is $L$. This should be compared to the main result of [14, Main Theorem], which concerns fluctuations in the self-intersection number for randomly closed curves on a surface, where sampling is by word length rather than geometric length. Let $\Upsilon$ be an orientable, compact surface with boundary and negative Euler characteristic $\chi$, and let $\mathscr{F}=\mathscr{F}_{\Upsilon}$ be its fundamental group. This is a free group on $2 g$ generators, where $g=2-2 \chi$. Each conjugacy class in $\mathscr{F}$ represents a free homotopy class of closed curves on $\Upsilon$. For each such conjugacy class $\alpha$ there is a well-defined word length $L=L(\alpha)$ (the minimal word length of a representative element) and a well-defined self-intersection count $N(\alpha)$ (the minimum number of transversal self-intersections of a closed curve in the free homotopy class). The main result of [14] states that if $\alpha$ is randomly chosen from among all conjugacy classes with word length $L$, then for certain positive constants $\kappa^{*}, \sigma^{*}$ depending on the Euler characteristic, as $L \rightarrow \infty$,

$$
\frac{N\left(\alpha_{L}\right)-\kappa_{\Upsilon}^{*} L^{2}}{\sigma^{*} L^{3 / 2}} \stackrel{D}{\longrightarrow} \Phi,
$$

where $\Phi$ is the standard unit Gaussian distribution. The methods of this paper can be adapted to show that the main result of [14] extends to compact surfaces without boundary and with genus $g \geq 2$. To reconcile this with Theorem 1.3 (which at first sight might appear to suggest that fluctuations should be on the order of $L$, not $L^{3 / 2}$ ), observe that when closed geodesics are randomly chosen according to word length $L$, the order of magnitude of fluctuations in geometric length is $L^{1 / 2}$; this is enough to increase the size of typical fluctuations in self-intersection count by a factor of $L^{1 / 2}$.

\section{Standing notation}

Throughout this paper, $p: S \Upsilon \rightarrow \Upsilon$ will denote the natural projection from the unit tangent bundle $S \Upsilon$ to the surface $\Upsilon$, and $\gamma(t)=\gamma(t ; v)$ will denote the orbit of the geodesic flow with initial tangent vector $v \in S \Upsilon$. The letter $\pi$ will be reserved for the semiconjugacy of flows constructed in Section 3 , and $\phi_{t}$ for the suspension flow in this construction. Finally, $\sigma$ will be used to denote the unilateral shift on any of the sequence spaces $\Sigma, \Sigma^{+}$, and so on, used in the symbolic dynamics.

\section{Intersection kernel}

The proofs of the main results will rely on the fact that the self-intersection counts are (in the terminology of [19]) " $U$-statistics", that is, functions that can be written in the form (10) below. In this section, we collect some essential properties of the kernel function $H_{\delta}$ in this representation, and then present a short heuristic argument that shows how the $U$-statistic representation can be used to reduce the weak convergence assertion (2) to Ratner's central limit theorem [34, Theorem 2.1] for the geodesic flow. 


\subsection{The intersection kernel}

Geodesics on any surface, regardless of its curvature, look locally like straight lines. Hence, for any compact surface $\Upsilon$ with smooth Riemannian metric there exists $\varrho>$ 0 such that, if $\alpha$ and $\beta$ are geodesic segments of length at most $\varrho$, then $\alpha$ and $\beta$ intersect transversally, if at all, in at most one point. It follows that for any geodesic segment $\gamma$ of length $T$, the self-intersection number $N(\gamma)=N_{T}(\gamma)$ can be computed by partitioning $\gamma$ into nonoverlapping segments of common length $\delta \leq \varrho$ and counting the number of pairs that intersect transversally. Let $\alpha_{i}$ and $\alpha_{j}$ be two such segments; then the event that these segments intersect is completely determined by their initial points and directions, as is the angle of intersection.

\section{Definition 2.1}

The intersection kernel $H_{\delta}: S \Upsilon \times S \Upsilon \rightarrow \mathbb{R}_{+}$is the nonnegative function that takes the value $H_{\delta}(u, v)=1$ if the geodesic segments of length $\delta$ with initial tangent vectors $u$ and $v$ intersect transversally, and $H_{\delta}(u, v)=0$ otherwise.

Assume henceforth that $\delta \leq \varrho$; then for any geodesic $\tilde{\gamma}$,

$$
N_{T}(\tilde{\gamma})=\frac{1}{2} \sum_{i=1}^{n} \sum_{j=1}^{n} H_{\delta}(\tilde{\gamma}(i \delta), \tilde{\gamma}(j \delta)) \text {. }
$$

The factor $1 / 2$ compensates for the double counting that results from letting both indices of summation $i, j$ range over all $n$ geodesic segments. The diagonal terms in this sum are all zero, because the segment $\gamma(i \delta)$ does not intersect itself transversally.

\subsection{The associated integral operators}

The intersection kernel $H_{\delta}(u, v)$ is symmetric in its arguments and Borel-measurable, but not continuous, because self-intersections can be created or destroyed by small perturbations of the initial vectors $u, v$. Nevertheless, $H_{\delta}$ induces a self-adjoint integral operator on the Hilbert space $L^{2}\left(v_{L}\right)$ by

$$
H_{\delta} \psi(u)=\int_{v \in S \Upsilon} H_{\delta}(u, v) \psi(v) d v_{L}(v) .
$$

LEMMA 2.2

For all sufficiently small $\delta>0$,

$$
H_{\delta} 1(u):=\int H_{\delta}(u, v) d v_{L}(v)=\delta^{2} \kappa
$$

for all $u \in M$. Thus, the constant function 1 is an eigenfunction of the operator $H_{\delta}$, and consequently the normalized kernel $H_{\delta}(u, v) / \delta^{2} \kappa_{\varphi}$ is a symmetric Markov kernel on $S \Upsilon \times S \Upsilon$. 


\section{Remark 2.3}

This result (simple though it may be) is the crucial geometric property of the intersection kernel. Clearly, the intersection kernel induces an integral operator on $L^{2}(\mu)$ for any finite measure $\mu$ on $S \Upsilon$ (just replace $\nu_{L}$ by $\mu$ in the definition (11)). But in general-and in particular, when $\mu \neq v_{L}$ is a Gibbs state-the constant function 1 will not be an eigenfunction of this operator.

Proof

Denote by $\gamma=\gamma([0, \delta] ; u)$ the geodesic segment of length $\delta$ with initial tangent vector $u$. For small $\delta>0$ and fixed angle $\theta$, the set of points $x \in \Upsilon$ such that a geodesic segment of length $\delta$ with initial base point $x$ intersects $\gamma$ at angle $\theta$ is approximately a rhombus of side $\delta$ with an interior angle $\theta$, with area $\delta^{2}|\sin \theta|$, and this approximation is asymptotically sharp as $\delta \rightarrow 0$. Consequently, as $\delta \rightarrow 0$,

$$
\int H_{\delta}(u, v) d v_{L}(v) \sim \delta^{2} \int_{0}^{2 \pi} \varphi(\theta)|\sin \theta| d \theta /(2 \pi|M|)=\delta^{2} \kappa_{\varphi},
$$

and the relation $\sim$ holds uniformly for $u \in S \Upsilon$.

It remains to show that the approximate equality $\sim$ is actually an equality for small $\delta>0$. Recall that for $\delta \leq \varrho$, any two distinct geodesic segments of length $\delta$ can intersect transversally at most once. Consider the geodesic segments of length $\delta$ with initial direction vectors $u$ and $v$. For any integer $m \geq 2$, each of these segments can be partitioned into $m$ nonoverlapping subsegments (each open on one end and closed on the other) of length $\delta / m$. At most one pair of these constituent subsegments can intersect; hence,

$$
H_{\delta}(u, v)=\sum_{i=0}^{m-1} \sum_{j=0}^{m-1} H_{\delta / m}(\tilde{\gamma}(i \delta ; u), \tilde{\gamma}(j \delta ; v)) .
$$

Integrating over $v$ with respect to the Liouville measure $v_{L}$ and using the invariance of $v_{L}$ relative to the geodesic flow, we obtain

$$
H_{\delta} 1(u)=\sum_{i=0}^{m-1} m H_{\delta / m} 1(\tilde{\gamma}(i \delta ; u)) .
$$

Let $m \rightarrow \infty$, and use the approximation (13) (with $\delta$ replaced by $\delta / m$ ); since this approximation holds uniformly, it follows that $H_{\delta} 1(u)=\delta^{2} \kappa_{S}$.

Lemma 2.2 is the only result from this section that will be needed for the proofs of the main results. The remainder of this section is devoted to a proof of the "law of large numbers" (1) and to a heuristic argument for Theorem 1.1 that is simpler and more illuminating than the formal proofs that will follow. 
LEMMA 2.4

For each sufficiently small $\delta>0$, the integral operator $H_{\delta}$ on $L^{2}\left(v_{L}\right)$ is compact.

Proof

If the kernel $H_{\delta}(u, v)$ were jointly continuous in its arguments $u, v$, then this would follow by standard results about integral operators (see, e.g., [41]). Since $H_{\delta}$ is not continuous, these standard results do not apply; nevertheless, the argument for compactness is elementary. It suffices to show that the mapping $u \mapsto H_{\delta}(u, \cdot)$ is continuous relative to the $L^{2}$-norm. Take $u, u^{\prime} \in S \Upsilon$, and let $\alpha, \alpha^{\prime}$ be the geodesic segments of length $\delta$ starting at $u, u^{\prime}$, respectively. If $u, u^{\prime}$ are close, then the geodesic segments $\alpha, \alpha^{\prime}$ are also close. Hence, for all but very small angles $\theta$ the set of points $x \in M$ such that a geodesic segment of length $\delta$ with initial base point $x$ intersects $\alpha$ at angle $\theta$ but does not intersect $\alpha^{\prime}$ is small. Consequently, the functions $H_{\delta}(u, \cdot)$ and $H_{\delta}\left(u^{\prime}, \cdot\right)$ differ on a set of small measure.

Lemma 2.4 implies that Hilbert-Schmidt theory (see [41]) applies. In particular, the nonzero spectrum of $H_{\delta}$ consists of isolated real eigenvalues $\lambda_{j}$ of finite multiplicity (and listed according to multiplicity). The corresponding (real) eigenfunctions $\psi_{j}$ can be chosen so as to constitute an orthonormal basis of $L^{2}\left(v_{L}\right)$, and the eigenvalue sequence $\lambda_{j}$ is square-summable.

\section{LEMMA 2.5}

The kernel $\bar{H}_{\delta}:=H_{\delta} / \delta^{2} \kappa_{\varphi}$ satisfies the Doeblin condition: there exist an integer $n \geq 1$ and a positive real number $\varepsilon$ such that

$$
\bar{H}_{\delta}^{(n)}(u, v) \geq \varepsilon \quad \text { for all } u, v \in S \Upsilon,
$$

where $H_{\delta}^{(n)}$ denotes the kernel of the $n$-fold iterated integral operator $H_{\delta}$.

\section{Proof}

Choose $n$ so large that for any two points $x, y \in \Upsilon$ there is a sequence $\left\{x_{i}\right\}_{0 \leq i \leq n}$ of $n+1$ points beginning with $x_{0}=x$ and ending at $x_{n}=y$, and such that each successive pair of consecutive points $x_{i}, x_{i+1}$ are at distance less than $\delta / 4$. Then for any two geodesic segments $\alpha, \beta$ of length $\delta$ on $S$ there is a chain of $n+1$ geodesic segments $\alpha_{i}$, all of length $\delta$, beginning at $\alpha_{0}=\alpha$ and ending at $\alpha_{n}=\beta$, such that any two successive segments $\alpha_{i}$ and $\alpha_{i+1}$ intersect transversally. Since the intersections are transversal, the initial points and directions of these segments can be jiggled slightly without undoing any of the transversal intersections. This implies (14). 
COROLLARY 2.6

The eigenvalue $\delta^{2} \kappa_{\varphi}$ is a simple eigenvalue of the integral operator $H_{\delta}$, and the rest of the spectrum lies in a disk of radius less than $\delta^{2} \kappa_{\varphi}$.

Proof

This is a standard result in the theory of Markov operators.

COROLLARY 2.7

For every $j \geq 2$, the eigenfunction $\psi_{j}$ has mean zero relative to $v_{L}$, and distinct eigenfunctions are uncorrelated.

\section{Proof}

The spectral theorem guarantees orthogonality of the eigenfunctions. The key point is that $\psi_{1}=1$ is an eigenfunction, and so the orthogonality $\psi_{j} \perp \psi_{1}$ implies that each $\psi_{j}$ for $j \geq 2$ has mean zero.

LEMMA 2.8

If $\delta>0$ is sufficiently small, then $H_{\delta}$ has eigenvalues other than zero and $\lambda_{1}(\delta)$.

\section{Proof}

Otherwise, the Markov operator $\bar{H}_{\delta}$ would be a projection operator: for every $\psi \in$ $L^{2}\left(v_{L}\right)$, the function $\bar{H}_{\delta} \psi$ would be constant. But if $\delta>0$ is small, this is obviously not the case.

\subsection{Law of large numbers}

The law of large numbers (1) for random geodesics can be deduced from Birkhoff's ergodic theorem using the representation (10) of the self-intersection count. The first step is to approximate the kernel $H_{\delta}$ by continuous kernels. Fix $0<\delta<\varrho$, where $\varrho>0$ is small enough that any two geodesic segments on the surface $\Upsilon$ of length $\varrho$ will intersect transversally at most once.

\section{LEMMA 2.9}

For each $\varepsilon>0$ there exist continuous functions $H_{\delta}^{-}, H_{\delta}^{+}: S \Upsilon \times S \Upsilon \rightarrow[0,1]$ such that $H_{\delta}^{-} \leq H_{\delta} \leq H_{\delta}^{+}$and such that for each $u \in S \Upsilon$,

$$
\int\left(H_{\delta}^{+}(u, v)-H_{\delta}^{-}(u, v)\right) d v_{L}(v)<\varepsilon .
$$

Proof

Fix $\varepsilon^{\prime}>0$ such that $\delta+2 \varepsilon^{\prime}<\varrho$, and let $\psi:[0,1] \rightarrow[0,1]$ be a continuous function 
such that $\psi(0)=\psi(1)=0$ and $\psi=1$ on the interval $\left[\varepsilon^{\prime}, 1-\varepsilon^{\prime}\right]$. For unit tangent vectors $u, v \in S \Upsilon$ such that the geodesic segments $\gamma_{u}, \gamma_{v}$ of length $\delta$ based at $u, v$ intersect at angle $\theta \in(0, \pi)$ at times $t_{u}, t_{v} \in[0, \delta]$, set

$$
H_{\delta}^{-}(u, v)=\psi(\theta / \pi) \psi\left(t_{u} / \delta\right) \psi\left(t_{v} / \delta\right)
$$

and for all other $u, v$ set $H_{\delta}^{-}(u, v)=0$. Similarly, for unit tangent vectors $u, v \in S \Upsilon$ such that the geodesic segments $\gamma_{u}, \gamma_{v}$ of length $\delta+2 \varepsilon^{\prime}$ based at $u, v$ intersect at times $t_{u}, t_{v} \in\left(-\varepsilon^{\prime}, \delta+\varepsilon^{\prime}\right)$, set

$$
H_{\delta}^{+}(u, v)=\psi\left(t_{u} /\left(\delta+2 \varepsilon^{\prime}\right)\right) \psi\left(t_{v} /\left(\delta+2 \varepsilon^{\prime}\right)\right),
$$

and for all other $u, v$, set $H_{\delta}^{+}(u, v)=0$. Clearly, $0 \leq H_{\delta}^{-} \leq H_{\delta} \leq H_{\delta}^{+}$, and by an argument like that in the proof of Lemma 2.2, it can be shown that if $\varepsilon^{\prime}>0$ is sufficiently small, then (15) will hold for all $u$.

PROPOSITION 2.10

Let $(\mathcal{X}, d)$ be a compact metric space, and let $K: \mathcal{X}^{2} \rightarrow \mathbb{R}$ be continuous. If $\mu$ is a Borel probability measure on $\mathcal{X}$ and $T: \mathcal{X} \rightarrow \mathcal{X}$ is an ergodic, measure-preserving transformation (not necessarily continuous) relative to $\mu$, then

$$
\lim _{n \rightarrow \infty} \frac{1}{n^{2}} \sum_{i=1}^{n} \sum_{j=1}^{n} K\left(T^{i} x, T^{j} x\right)=\iint_{X \times X} K(y, z) d \mu(y) d \mu(z)
$$

for $\mu$-almost every $x$.

\section{Proof}

The function $K$ is bounded, since it is continuous, so the double integral in (16) is well defined and finite. Furthermore, the set of functions $K_{x}$ defined by $K_{x}(y):=K(x, y)$, where $x$ ranges over the space $\mathcal{X}$, is equicontinuous, and the function

$$
\bar{K}_{x}:=\int_{X} K_{x}(y) d \mu(y)
$$

is continuous in $x$. The equicontinuity of the functions $K_{x}$ implies, by the ArzelàAscoli theorem, that for any $\varepsilon>0$ there is a finite subset $F_{\varepsilon}=\left\{x_{i}\right\}_{1 \leq i \leq I}$ such that for any $x \in S M$ there is at least one index $i \leq I$ such that

$$
\left\|K_{x}-K_{x_{i}}\right\|_{\infty}<\varepsilon
$$

It follows that the time average of $K_{x}$ along any trajectory differs from the corresponding time average of $K_{x_{i}}$ by less than $\varepsilon$. Since the set $F_{\varepsilon}$ is finite, Birkhoff's 
theorem implies that, for $\mu$-almost every $x \in \mathcal{X}$,

$$
\lim _{n \rightarrow \infty} \frac{1}{n} \sum_{j=1}^{n} K\left(y, T^{j} x\right)=\int K\left(y, x^{\prime}\right) d \mu\left(x^{\prime}\right) \quad \text { for each } y \in F_{\varepsilon} .
$$

Consequently, it follows from equicontinuity (let $\varepsilon \rightarrow 0$ ) and the continuity in $x$ of the averages $\bar{K}_{x}$ that almost surely

$$
\lim _{n \rightarrow \infty} \frac{1}{n} \sum_{j=1}^{n} K\left(y, T^{j} x\right)=\int K(y, x) d \mu(y)
$$

uniformly for all $y \in \mathcal{X}$. The uniformity of this convergence guarantees that (16) holds $\mu$-almost surely.

\section{Proof of the strong law of large numbers (1)}

Let $H_{\delta}^{+}$and $H_{\delta}^{-}$be as in the statement of Lemma 2.9. By Proposition 2.10, for $\nu_{L^{-}}$ almost every $u \in S \Upsilon$,

$$
\lim _{n \rightarrow \infty} \frac{1}{n^{2}} \sum_{i=1}^{n} \sum_{j=1}^{n} H_{\delta}^{ \pm}\left(\tilde{\gamma}_{u}(i \delta), \tilde{\gamma}_{u}(j \delta)\right)=\int H_{\delta}^{ \pm}(v, w) d v_{L}(v) d v_{L}(w) .
$$

Hence, by Lemma 2.9 (with $\varepsilon^{\prime} \rightarrow 0$ ) and Lemma 2.2, for $v_{L}$-almost every $u \in S \Upsilon$,

$$
\lim _{n \rightarrow \infty} \frac{1}{n^{2}} \sum_{i=1}^{n} \sum_{j=1}^{n} H_{\delta}\left(\gamma_{u}(i \delta), \gamma_{u}(j \delta)\right)=\int H_{\delta}(v, w) d v_{L}(v) d v_{L}(w)=\delta^{2} \kappa
$$

This proves that (1) holds for $t \rightarrow \infty$ along the sequence $t=n \delta$. Since $\delta>0$ is arbitrary, and since the self-intersection counts are obviously monotone in $t$, relation (1) follows.

\subsection{Weak convergence: heuristics}

The results of Sections 2.1 and 2.2 can be used to give a compelling-but nonrigorous-explanation of the weak convergence asserted in Theorem 1.1. The HilbertSchmidt theorem asserts that a symmetric integral kernel in the class $L^{2}\left(v_{L} \times v_{L}\right)$ has an $L^{2}$-convergent eigenfunction expansion. The intersection kernel $H_{\delta}(u, v)$ meets the requirements of this theorem, and so its eigenfunction expansion converges in $L^{2}\left(v_{L} \times v_{L}\right)$ :

$$
H_{\delta}(u, v)=\sum_{k=1}^{\infty} \lambda_{k} \psi_{k}(u) \psi_{k}(v)
$$


The $L^{2}$-convergence of the series does not, of course, imply pointwise convergence; this is why the following argument is not a proof. Nevertheless, let us proceed formally, ignoring convergence issues. Recall (Corollary 2.7) that the eigenfunctions are mutually uncorrelated, and so all except the constant eigenfunction $\psi_{1}$ have mean zero relative to $v_{L}$. Thus, the representation (10) of the intersection number $N_{\varphi}(n \delta)$ can be rewritten as follows:

$$
\begin{aligned}
N_{\varphi}(n \delta)-(n \delta)^{2} \kappa_{g} & =\frac{1}{2} \sum_{i=1}^{n} \sum_{j=1}^{n} H_{\delta}(\tilde{\gamma}(i \delta), \tilde{\gamma}(j \delta))-(n \delta)^{2} \kappa_{g} \\
& =\frac{1}{2} \sum_{i=1}^{n} \sum_{j=1}^{n} \sum_{k=2}^{\infty} \lambda_{k}(\delta) \psi_{k}(\tilde{\gamma}(i \delta)) \psi_{k}(\tilde{\gamma}(j \delta)) \\
& =\frac{1}{2} \sum_{k=2}^{\infty} \lambda_{k}(\delta)\left(\sum_{i=1}^{n} \psi_{k}(\tilde{\gamma}(i \delta))\right)^{2} .
\end{aligned}
$$

If the eigenfunctions $\psi_{j}$ were Hölder-continuous, the central limit theorem for the geodesic flow in [34] would imply that, for any finite $K$, the joint distribution of the random vector

$$
\left(\frac{1}{\sqrt{n}} \sum_{i=1}^{n} \psi_{k}(\tilde{\gamma}(i \delta))\right)_{2 \leq k \leq K}
$$

converges, as $n \rightarrow \infty$, to a (possibly degenerate) $K$-variate Gaussian distribution centered at the origin. (The central limit theorem in [34] is stated only for the case $K=1$, but the general case follows by standard weak convergence arguments; see CramerWold device in [4, Chapter 1].) Hence, for every $K<\infty$, the distribution of the truncated sum

$$
\frac{1}{n} \sum_{k=2}^{K} \lambda_{k}(\delta)\left(\sum_{i=1}^{n} \psi_{k}(\tilde{\gamma}(i \delta))\right)^{2}
$$

should converge, as $n \rightarrow \infty$, to that of a quadratic form in the entries of the limiting Gaussian distribution. ${ }^{\dagger}$

\footnotetext{
${ }^{\dagger}$ This would follow by the spectral theorem for symmetric matrices and elementary properties of the multivariate Gaussian distribution. To see this, suppose that the limit distribution of the random vector (19) is meanzero Gaussian with (possibly degenerate) covariance matrix $\Sigma$; this distribution is the same as that of $\Sigma^{1 / 2} Z$, where $Z$ is a Gaussian random vector with mean zero and identity covariance matrix. Let $\Lambda$ be the diagonal matrix with diagonal entries $\lambda_{j}(\delta)$. Then the limit distribution of (20) is identical to that of $Z^{T} M Z$, where $M=\Sigma^{1 / 2} \Lambda \Sigma^{1 / 2}$. But the matrix $M$ is symmetric, so it may be factored as $M=U^{T} D U$, where $U$ is an orthogonal matrix and $D$ is diagonal. Now if $Z$ is mean-zero Gaussian with the identity covariance matrix, then so is $U Z$, since $U$ is orthogonal. Thus, $Z^{T} M Z$ is a quadratic form in independent, standard normal random variables.
} 
Unfortunately, it seems that there is no way to make this argument rigorous, because there is no obvious way to show that the series (17) converges pointwise. (If the kernel $H_{\delta}$ were nonnegative semidefinite, then Mercer's theorem might be applied, in conjunction with a smoothing argument; however, $H_{\delta}$ is in general not nonnegative semidefinite.) Thus, it will be necessary to proceed by a more indirect route, via symbolic dynamics and thermodynamic formalism.

\section{Symbolic dynamics}

\subsection{Shifts and suspension flows}

Symbolic dynamics provides an approach to the study of hyperbolic flows that transforms questions about orbits of the flow to equivalent (or nearly equivalent) questions about a shift of finite type. The shift of finite type (either one-sided or two-sided) on a finite alphabet $\mathcal{A}$ with transition matrix $A: \mathcal{A} \times \mathcal{A} \rightarrow\{0,1\}$ is the system $\left(\Sigma^{+}, \sigma\right)$ or $(\Sigma, \sigma)$, where

$$
\begin{aligned}
\Sigma^{+} & =\left\{\left(x_{n}\right)_{n \geq 0} \in \mathcal{A}^{\mathbb{Z}}+\mid A\left(x_{n}, x_{n+1}\right)=1 \forall n \geq 0\right\} \quad \text { and } \\
\Sigma & =\left\{\left(x_{n}\right)_{n \in \mathbb{Z}} \in \mathcal{A}^{\mathbb{Z}} \mid A\left(x_{n}, x_{n+1}\right)=1 \forall n \in \mathbb{Z}\right\},
\end{aligned}
$$

and $\sigma$ is the forward shift operator on sequences. (Equivalently, one can define a shift of finite type to be the forward shift operator acting on the space of all sequences with entries in $\mathcal{A}$ in which certain subwords of a certain length $r$ do not occur.) If there exists $m \geq 1$ such that $A^{m}$ has strictly positive entries, then the corresponding shifts are topologically mixing relative to the usual topology on $\Sigma$ or $\Sigma^{+}$, which is metrizable by

$$
d(x, y)=2^{-n(x, y)}
$$

where $n(x, y)$ is the minimum nonnegative integer $n$ such that $x_{j} \neq y_{j}$ for $j= \pm n$.

For a continuous function $F: \Sigma \rightarrow(0, \infty)$ on $\Sigma$ (or on $\Sigma^{+}$), the suspension flow under $F$ is a flow $\phi_{t}$ on the space

$$
\Sigma_{F}:=\{(x, t): x \in \Sigma \text { and } 0 \leq t \leq F(x)\}
$$

with points $(x, F(x))$ and $(\sigma x, 0)$ identified. In the suspension flow an orbit beginning at some point $(x, s)$ moves at unit speed up the fiber over $(x, 0)$ until reaching the roof $(x, F(x))$, at which time it jumps to the point $(\sigma x, 0)$ and then proceeds up the fiber over $(\sigma x, 0)$, that is,

$$
\begin{aligned}
& \phi_{t}(x, s)=(x, s+t) \quad \text { if } s+t \leq F(x) \\
& \phi_{t}(x, s)=\phi_{t-F(x)+s}(\sigma x, 0) \quad \text { otherwise. }
\end{aligned}
$$


We will use the notation

$$
\begin{aligned}
& \mathcal{F}_{x}:=\{(x, s): s \in[0, F(x))\} \quad \text { and } \\
& \mathcal{F}_{x}^{r}:=\phi_{r}\left(\mathcal{F}_{x}\right)
\end{aligned}
$$

for fibers and their time shifts. An orbit of the suspension flow is periodic if and only if it passes through a point $(x, 0)$ such that $x$ is a periodic sequence; if the minimal period of the sequence $x$ is $n$, then the minimal period of the corresponding periodic orbit of $\phi_{t}$ is the sum of the lengths of the fibers visited by the orbit, which is given by

$$
S_{n} F(x):=\sum_{j=0}^{n-1} F\left(\sigma^{j} x\right) .
$$

The term symbolic dynamics is used loosely to denote a coding of orbits of a flow by elements $x \in \Sigma$ of a shift of finite type. In the case of a hyperbolic flow, this coding extends to a Hölder-continuous ${ }^{\dagger}$ semiconjugacy with a suspension flow over a shift of finite type with a Hölder-continuous height function $F$. Existence of such semiconjugacies was proved in general for Axiom A flows by Bowen [10], and by Ratner [35] for geodesic flows on negatively curved surfaces.

PROPOSITION 3.1 ([35, Theorems 2.1, 3.1, 4.1], [10])

For the geodesic flow $\gamma_{t}$ on any compact surface $\Upsilon$ with a Riemannian metric of negative curvature there exists a suspension flow $\left(\Sigma_{F}, \phi_{t}\right)$ over a shift of finite type and a Hölder-continuous surjection $\pi: \Sigma_{F} \rightarrow S \Upsilon$ such that

$$
\pi \circ \phi_{t}=\gamma_{t} \circ \pi \quad \text { for all } t \in \mathbb{R} \text {. }
$$

The suspension flow $\left(\Sigma_{F}, \phi_{t}\right)$ and the projection $\pi$ can be chosen in such a way that the following properties hold.

(A) For some $N<\infty$, the mapping $\pi$ is at most $N$-to- 1 .

(B) The suspension flow and the geodesic flow have the same topological entropy $\theta>0$.

(C) For some $\varepsilon>0$,

(i) the projection $\pi$ is $\mu$-almost surely one-to-one for every Gibbs state $\mu$ with entropy larger than $\theta-\varepsilon$, and

\footnotetext{
${ }^{\dagger}$ The implied metric on the suspension space $\Sigma_{F}$ is the taxicab metric induced by the flow $\phi_{t}$ and the metric $d$ on $\Sigma$ specified above-see [13] for details. Roughly, the distance between two points in the suspension space is the length of the shortest path between them that consists of finitely many segments along flow lines and finitely many segments of the form $\left[(x, s),\left(x^{\prime}, s\right)\right]$. The metric on $S \Upsilon$ is the metric induced by the Riemannian metric on $T \Upsilon$ (see, e.g., [32]).
} 
(ii) the number $M(t)$ of closed geodesics with more than one $\pi$-preimage and prime period at most $t$ satisfies

$$
\limsup _{t \rightarrow \infty} t^{-1} \log M(t) \leq \theta-\varepsilon
$$

For the definition of a Gibbs state, see Section 4 below; both the Liouville measure and the maximum entropy-invariant measure are Gibbs states. The conclusions (C)(i) and (C)(ii) are not explicitly stated in [10], but both follow from Bowen's construction. (See [30, Section 3] for further discussion of this point.) Finally, observe that if $\pi$ is a semiconjugacy as in Proposition 3.1, then so is the mapping $\pi_{s}=\pi \circ \phi_{s}$, for any $s \in \mathbb{R}$. (See Remark 3.8 for implications of this.)

\subsection{Series's construction}

For the geodesic flow on a compact surface of constant negative curvature, a different symbolic dynamics was constructed by Series in [38] (see also [12], and for related constructions [2] and [24]). This construction is better suited to enumeration of selfintersections. In this section, we give a résumé of some of the important features of Series's construction.

Assume first that $\Upsilon$ has constant curvature -1 and genus $g \geq 2$. Then the universal covering space of $\Upsilon$ is the hyperbolic plane $\mathbb{D}$, realized as the unit disk with the usual (Poincaré) metric. The fundamental group $\Gamma=\pi_{1}(\Upsilon)$ is a discrete, finitely generated, cocompact group of isometries of $\mathbb{D}$. Thus, $\Upsilon$ can be identified with $\mathbb{D} / \Gamma$. This in turn can be identified with a fundamental polygon $\mathcal{P}$, with compact closure in $\mathbb{D}$, whose sides are geodesic segments in $\mathbb{D}$ that are paired by elements in a (symmetric) generating set for $\Gamma$. The polygon $\mathcal{P}$ can be chosen in such a way that the even corners condition is satisfied: that is, each geodesic arc in $\partial \mathcal{P}$ extends to a complete geodesic in $\mathbb{D}$ that is completely contained in $\bigcup_{g \in \Gamma} g(\partial \mathcal{P})$. The geodesic lines in $\bigcup_{g \in \Gamma} g(\partial \mathcal{P})$ project to closed geodesics in $\Upsilon$; because the polygon $\mathcal{P}$ has only finitely many sides, there are only finitely many such projections. Call these the boundary geodesics.

Except for those vectors tangent to one of the boundary geodesics, each unit tangent vector $v \in S \Upsilon$ can be uniquely lifted to the unit tangent bundle $S \mathbb{D}$ of the hyperbolic plane in such a way that either the lifted vector $L(v)$ has base point in the interior of $\mathcal{P}$, or lies on the boundary of $\mathcal{P}$ but points into $\mathcal{P}$. The vector $L(v)$ uniquely determines a geodesic line in $D$, with initial tangent vector $L(v)$, which converges to distinct points on the circle at infinity as $t \rightarrow \pm \infty$. The mapping $L$ : $S \Upsilon \rightarrow S \mathbb{D}$ is smooth except at those vectors that lift to vectors tangent to one of the boundary geodesics; at these vectors, $L$ is necessarily discontinuous. Denote by $\Xi \subset S \Upsilon$ the set of all vectors $v$ such that $L(v)$ is based at a point on the boundary of $\mathcal{P}$. 
For any shift $\left(\Sigma^{+}, \sigma\right)$, any $x \in \Sigma$ or $\Sigma^{+}$, and any subset $J \subset \mathbb{Z}_{+}$, let $\Sigma_{J}^{+}(x)$ be the cylinder set consisting of all $y \in \Sigma^{+}$such that $x_{j}=y_{j}$ for all $j \in J$. For any sequence $x \in \Sigma$, denote by

$$
x^{+}=x_{0} x_{1} x_{2} \cdots \quad \text { and } \quad x^{-}=x_{-1} x_{-2} x_{-3} \cdots
$$

the forward and backward coordinate subsequences. The sequence $x^{-}$need not be an element of $\Sigma^{+}$, since its coordinates are reversed. Let $\Sigma^{-}$be the set of all $x^{-}$such that $x \in \Sigma$; then $\left(\Sigma^{-}, \sigma\right)$ is a shift of finite type (whose transition matrix $A^{\dagger}$ is the transpose of $A$ ).

PROPOSITION 3.2 ([38, Theorem 3.1 and Section 4])

Let $\Upsilon$ be a compact surface equipped with a Riemannian metric of constant curvature -1 . There exist a shift $(\Sigma, \sigma)$ of finite type, a suspension flow $\left(\Sigma_{F}, \phi_{t}\right)$ over the shift, and surjective, Hölder-continuous mappings $\xi_{ \pm}: \Sigma^{ \pm} \rightarrow \partial \mathbb{D}$ and $\pi: \Sigma_{F} \rightarrow S \Upsilon$ such that $\pi$ is a semiconjugacy with the geodesic flow (i.e., equation (22) holds), and such that the following properties hold:

(A) $\Xi=\pi(\Sigma \times\{0\})$;

(B) the endpoints on $\partial \mathbb{D}$ of the geodesic with initial tangent vector $L \circ \pi(x, 0)$ are $\xi_{ \pm}\left(x^{ \pm}\right)$

(C) $\quad F(x)$ is the time taken by this geodesic line to cross $\mathcal{P}$.

Furthermore, the maps $\xi_{ \pm}$send cylinder sets $\Sigma_{[0, m]}^{ \pm}(x)$ onto closed arcs $J_{m}^{ \pm}\left(x^{ \pm}\right)$such that, for certain constants $C<\infty$ and $0<\beta_{1}<\beta_{2}<1$ independent of $m$ and $x$,

(D) the lengths of $J_{m}^{ \pm}\left(x^{ \pm}\right)$are between $C \beta_{1}^{m}$ and $C \beta_{2}^{m}$, and

(E) distinct arcs $J_{m}^{+}\left(x^{+}\right)$and $J_{m}^{+}\left(y^{+}\right)$of the same generation $m$ have disjoint interiors (and similarly when + is replaced by - ).

Consequently, the semiconjugacy $\pi$ fails to be one-to-one only for geodesics whose lifts to $\mathbb{D}$ have at least one endpoint that is an endpoint of some arc $J_{m}^{ \pm}(x)$. Finally, all but finitely many closed geodesics (the boundary geodesics) correspond uniquely to periodic orbits of the suspension flow, and for each nonexceptional closed geodesic the length of the representative sequence in $\Sigma$ is the word length of the free homotopy class relative to the standard generators of $\pi_{1}(\Upsilon)$.

(See also [12].) The last point is important because it implies that the set of geodesics where the semiconjugacy fails to be bijective is of first category, and has measure zero under any Gibbs state (in particular, under the Liouville and maximum entropy measures).

Series's construction relies heavily on the hypothesis that the underlying metric on $\Upsilon$ is of constant negative curvature. However, the key features of her construction carry over to metrics of variable negative curvature by virtue of the conformal 
equivalence theorem (see, e.g., [37, Chapter V]) for negatively curved Riemannian metrics on surfaces and by virtue of the structural stability theorem for Anosov flows (see [3], [28], [15]). Structural stability applies only to small perturbations of Anosov flows, and only geodesic flows on negatively curved surfaces are Anosov, so to use structural stability globally for geodesic flows, we must be able to show that there is a deformation (homotopy) taking one Riemannian metric to another through metrics of negative curvature. The following easy proposition states that for surfaces, conformal equivalence of negatively curved metrics implies the existence of a smooth deformation. (See [18] for a generalization to higher dimensions.)

PROPOSITION 3.3

Let $\varrho_{0}, \varrho_{1}$ be $C^{\infty}$-Riemannian metrics on $\Upsilon$, both with everywhere negative scalar curvatures. Then there exists a $C^{\infty}$-deformation $\left\{\varrho_{t}\right\}_{t \in[0,1]}$ through Riemannian metrics with everywhere negative scalar curvatures.

Proof

The conformal equivalence theorem (see [37, Chapter V, Theorem 1.3]) implies that there exists a strictly positive $C^{\infty}$-function $r=e^{2 u}$ on $\Upsilon$ such that $\varrho_{1}=r \varrho_{0}$. The scalar curvatures $K_{0}, K_{1}$ are related by the equation

$$
K_{1}=e^{-2 u}\left(K_{0}-2 \Delta u\right)
$$

where $\Delta$ is the Laplace-Beltrami operator with respect to $\varrho_{0}$. Since $K_{0}$ and $K_{1}$ are both negative everywhere, it follows that $K_{0}-t \Delta u<0$ for every $t \in[0,1]$. Thus, if $\varrho_{t}:=e^{-2 t u} \varrho_{0}$, then the curvature $K_{t}=e^{-2 t u}\left(K_{0}-t \Delta u\right)$ is everywhere negative, for every $t$.

Fix Riemannian metrics $\varrho_{0}$ and $\varrho_{1}$ of negative curvature on $\Upsilon$ such that $\varrho_{0}$ has constant curvature -1 , and let $\varrho_{s}$ be a smooth deformation as in Proposition 3.3. The geodesic flow on $S \Upsilon$ with respect to any Riemannian metric $\varrho_{s}$ of negative curvature is Anosov, and if the metrics $\varrho_{s}$ vary smoothly with $s$, then so do the vector fields of their geodesic flows. Hence, by the structural stability theorem, for each $s \in[0,1]$ there exists a Hölder-continuous homeomorphism $\Phi_{S}: S \Upsilon \rightarrow S \Upsilon$ that maps $\varrho_{0}$ geodesics to $\varrho_{s}$-geodesics. The Hölder exponent is constant in $s$, and the homeomorphisms $\Phi_{s}$ vary smoothly with $s$ in the Hölder topology (see [15]). Consequently, the homotopy $\Phi_{s}$ lifts to a homotopy $\tilde{\Phi}_{s}: S \mathbb{D} \rightarrow S \mathbb{D}$ of Hölder-continuous homeomorphisms of the universal covering space. Each homeomorphism $\tilde{\Phi}_{s}$ maps $\varrho_{0}$-geodesics to $\varrho_{s}$-geodesics, and for each $\varrho_{0}$-geodesic $\gamma$ the corresponding $\varrho_{s}$-geodesic $\tilde{\Phi}_{s}(\gamma)$ converges to the same endpoints on the circle at infinity $\partial \mathbb{D}$ as does $\gamma$.

Series's construction gives a semiconjugacy $\pi_{0}$ of a suspension flow $\left(\Sigma_{F_{0}}, \phi_{t}\right)$ with the $\varrho_{0}$-geodesic flow on $S \Upsilon$ that is nearly one-to-one in the senses described 
in Proposition 3.2. We have just seen that there is a homotopy of Hölder-continuous homeomorphisms $\Phi_{s}: S \Upsilon \rightarrow S \Upsilon$ such that each $\Phi_{s}$ maps $\varrho_{0}$-geodesics to $\varrho_{s}$-geodesics. Set $\Phi=\Phi_{1}$; because $\Phi$ is Hölder, it lifts to the suspension flow: in particular, there exist Hölder-continuous $F_{1}: \Sigma \rightarrow(0, \infty)$ and $\pi_{1}: \Sigma_{F_{1}} \rightarrow S \Upsilon$ and a Hölder-continuous homeomorphism $\Psi: \Sigma_{F_{0}} \rightarrow \Sigma_{F_{1}}$ that maps fibers of $\Sigma_{F_{0}}$ homeomorphically onto fibers of $\Sigma_{F_{1}}$ and that satisfies the conditions

$$
\begin{aligned}
& \Psi(x, 0)=(x, 0) \text { for every } x \in \Sigma, \text { and } \\
& \pi_{1} \circ \Psi=\Phi \circ \pi_{0} .
\end{aligned}
$$

Thus, the projection $\pi_{1}: \Sigma_{F_{1}} \rightarrow S \Upsilon$ is a semiconjugacy between the suspension flow on $\Sigma_{F_{1}}$ and the geodesic flow on $S \Upsilon$ relative to the metric $\varrho_{1}$.

\section{COROLLARY 3.4}

For any negatively curved Riemannian metric $\varrho_{1}$ on a compact surface $\Upsilon$, the suspension flow $\left(\Sigma_{F_{1}}, \phi_{t}\right)$ and the semiconjugacy $\pi_{1}: \Sigma_{F_{1}} \rightarrow S \Upsilon$ in Proposition 3.1 can be chosen in such a way that $\pi$ is one-to-one except on a set of first category, and only finitely many closed geodesics have more than one preimage.

\subsection{Symbolic dynamics and self-intersection counts}

For surfaces of constant curvature -1 , the symbolic dynamics have the convenient property that the geodesic segments $p \circ \pi\left(\mathscr{F}_{x}\right)$ and $p \circ \pi\left(\mathcal{F}_{y}\right)$ corresponding to two distinct fibers of the suspension flow can intersect at most once, since each is a single crossing of the fundamental polygon. Since it will be easiest for us to count selfintersections of a long geodesic by counting pairs of fibers whose images cross, we begin by recording a simple modification of the symbolic dynamics that guarantees only single crossings.

\section{LEMMA 3.5}

Given any sufficiently small $\varepsilon>0$, we can assume, without loss of generality, that the suspension flow has been chosen in such a way that the height function $F$ satisfies $0<F \leq \varepsilon$.

\section{Proof}

This can be arranged by a simple refinement of the symbolic dynamics constructed above. First, consider the suspension flow obtained by cutting the sections of the flow space lying above particular initial symbols $x_{0}=a$ into boxes, refining the alphabet so that there is one symbol per box, and adjusting the transition rule and the height function accordingly. In detail, fix an integer $m \geq 1$, replace the original alphabet $\mathcal{A}$ by the augmented alphabet $\mathcal{A}^{*}:=\mathcal{A} \times[m]$, where $[m]=\{1,2, \ldots, m\}$, and replace 
the transition matrix $A$ by the matrix $A^{*}$ defined by

$$
\begin{aligned}
A^{*}\left((a, j),\left(a^{\prime}, j^{\prime}\right)\right) & =1 \quad \text { if } a=a^{\prime} \text { and } j^{\prime}=j+1 \leq m, \\
& =1 \quad \text { if } A\left(a, a^{\prime}\right)=1 \text { and } j^{\prime}=1, j=m, \\
& =0 \quad \text { otherwise. }
\end{aligned}
$$

Define the shift $\left(\Sigma^{*}, \sigma^{*}\right)$ on the enlarged alphabet, with transition matrix $A^{*}$, accordingly. Let $v: \mathcal{A}^{*} \rightarrow \mathcal{A}$ be the projection on the first coordinate, and let $v^{*}: \Sigma^{*} \rightarrow$ $\Sigma$ be the induced projection of the corresponding sequence spaces. Finally, define $F^{*}: \Sigma^{*} \rightarrow(0, \infty)$ by $F^{*}\left(x^{*}\right)=F\left(v^{*}\left(x^{*}\right)\right) / m$. Then the mapping $p^{*}: \Sigma_{F *}^{*} \rightarrow \Sigma_{F}$ defined by

$$
p^{*}((x, j), s)=\left(x, s+(j-1) F^{*}(x)\right)
$$

provides a conjugacy between the suspension flow $\left(\Sigma_{F *}^{*}, \phi_{t}^{*}\right)$ with $\left(\Sigma_{F}, \phi_{t}\right)$. By choosing $m$ large, we can arrange that $F^{*}<\varepsilon$.

Unfortunately, this construction introduces periodicity into the underlying shift $\left(\Sigma^{*}, \sigma\right)$. This is a nuisance, because the basic results of thermodynamic formalism (see [11], [31]) that we will need later, including the central limit theorem (see [34]), require that the underlying shift be topologically mixing. But a simple modification of the foregoing construction can be used to destroy the periodicity. Choose one symbol $a^{\boldsymbol{\alpha}} \in \mathcal{A}$, and for this symbol only, cut the section of the flow space $\Sigma_{F}$ over $a^{\boldsymbol{\alpha}}$ into $m+1$ boxes, instead of the $m$ used in the construction above. Adjust the transition rule $A^{*}$, the height function $F$, and the projection mapping $p^{*}$ in the obvious manner to obtain a suspension flow conjugate to the original flow. The underlying shift for this modified suspension will be aperiodic, by virtue of the fact that the original shift $(\Sigma, \sigma)$ is aperiodic.

Observe that in both of these constructions, the cylinder sets of the modified shift $\left(\Sigma^{*}, \sigma^{*}\right)$ are contained in cylinder sets of $(\Sigma, \sigma)$ of comparable length (i.e., within a factor $m+1)$. Since, in Series's symbolic dynamics cylinder, sets of length $n$ correspond to boundary arcs in $\delta \mathbb{D}$ with lengths exponentially decaying in $n$, the same will be true for the modified symbolic dynamics.

By virtue of this lemma, we can assume without loss of generality that the suspension flow has been chosen in such a way that the images (under the projection $p \circ \pi$ ) of any two distinct fibers $\mathcal{F}_{x}$ and $\mathcal{F}_{y}$ intersect at most once in $\Upsilon$. Thus, the number of self-intersections of any geodesic segment can be computed by partitioning the segment into the images of successive fibers (the first and last segment will only represent partial fibers) and counting how many pairs intersect. With this in mind, define $h: \Sigma \times \Sigma \rightarrow\{0,1\}$ by setting $h(x, y)=1$ if the fibers $\mathcal{F}_{x}$ and $\mathcal{F}_{y}$ over $x$ and 
$y$ project to geodesic segments on $\Upsilon$ that intersect (transversally), and $h(x, y)=0$ if not. Clearly, for any periodic sequence $x \in \Sigma$ with minimal period $m$, the image (under $p \circ \pi$ ) of the periodic orbit of the suspension flow containing the point $(x, 0)$ will be a closed geodesic $\gamma$ with self-intersection count

$$
N(\gamma)=\frac{1}{2} \sum_{i=1}^{m} \sum_{j=1}^{m} h\left(\sigma^{i} x, \sigma^{j} x\right) .
$$

The function $h$ is not continuous, because a small change in the endpoints or directions of two intersecting geodesic segments can destroy the intersection. Nevertheless, for "most" sequences $x, y \in \Sigma$, a "small" number of coordinates $x_{j}, y_{j}$ will determine whether or not the geodesic segments corresponding to the fibers $\mathcal{F}_{x}$ and $\mathscr{F}_{y}$ intersect. Here is a way to make this precise. For each $m \geq 1$ and each sequence $x \in \Sigma$, denote by $\Sigma_{[-m, m]}(x)$ the cylinder set consisting of all $y \in \Sigma$ that agree with $x$ in all coordinates $-m \leq j \leq m$. For any two sequences $x, y \in \Sigma$ such that the geodesic segments $p \circ \pi\left(\mathscr{F}_{x}\right)$ and $p \circ \pi\left(\mathscr{F}_{y}\right)$ intersect, let $m(x, y)$ be the least nonnegative integer with the following property: for every pair of sequences $x^{\prime}, y^{\prime}$ such that $x^{\prime} \in \Sigma_{[-m, m]}(x)$ and $y^{\prime} \in \Sigma_{[-m, m]}(y)$, the geodesic segments $p \circ \pi\left(\mathcal{F}_{x^{\prime}}\right)$ and $p \circ \pi\left(\mathcal{F}_{y^{\prime}}\right)$ intersect, where $\mathcal{F}_{x^{\prime}}$ and $\mathcal{F}_{y^{\prime}}$ are the fibers of the suspension space over $x^{\prime}$ and $y^{\prime}$, respectively. For sequences $x, y$ such that the segments $p \circ \pi\left(\mathscr{F}_{x}\right)$ and $p \circ \pi\left(\mathcal{F}_{y}\right)$ do not intersect, set $m(x, y)=-1$. Define

$$
\begin{aligned}
h_{m}(x, y)=1 & \text { if } m(x, y)=m \geq 0, \\
=0 & \text { otherwise. }
\end{aligned}
$$

LEMMA 3.6

The function $h$ decomposes as

$$
h(x, y)=\sum_{m=0}^{\infty} h_{m}(x, y)+h_{\infty}(x, y),
$$

where $h_{\infty}(x, y) \neq 0$ (in which case $h_{\infty}(x, y)=1$ ) only if the geodesic segments $p \circ \pi\left(\mathcal{F}_{x}\right)$ and $p \circ \pi\left(\mathcal{F}_{y}\right)$ intersect at an endpoint of one of the two segments. The functions $h_{m}$ satisfy the following properties:

(A1) $h_{m}(x, y)$ depends only on the coordinates $x_{i}, y_{i}$ with $|i| \leq m$;

(A2) $h_{m}(x, y) \neq 0$ for at most one $m$; and

(A3) for some $0<\varrho<1$ and $C<\infty$ not depending on $n$, if $\sum_{m \geq n} h_{m}(x, y)+$ $h_{\infty}(x, y) \neq 0$, then the geodesics corresponding to the orbits of the suspension flow through $(x, 0)$ and $(y, 0)$ intersect either

(a) at an angle less than $C \varrho^{n}$, or 
(b) at a point at distance less than $C \varrho^{n}$ from one of the endpoints of one of the segments $p \circ \pi\left(\mathcal{F}_{x}\right)$ or $p \circ \pi\left(\mathcal{F}_{y}\right)$.

\section{Proof}

Only statement (A3) is nontrivial. Since the projection $\pi: \Sigma_{F} \rightarrow S \Upsilon$ is Höldercontinuous, it suffices to prove that for all large $n$, if two geodesic segments intersect at an angle larger than $C \varrho^{n}$ and at a point at distance greater than $C \varrho^{n}$ from any of the endpoints, then so will two geodesic segments of the same length whose initial points/directions are within distance $C \varrho^{2 n}$ of the initial points/directions of the original pair of geodesic segments. This holds because at small distances, geodesic segments on $\Upsilon$ look like straight line segments in the tangent space (to the intersection point).

A formula similar to (25) holds for the self-intersection count $N(t)=N(t ; \gamma)$ of an arbitrary geodesic segment $\gamma[0, t]$ (which, in general, will not be a closed curve). As in the case of closed geodesics, the self-intersection count can be computed by partitioning the segment into the images of successive fibers and counting how many pairs intersect. However, for arbitrary geodesic segments, the first and last segment will only represent partial fibers, and so intersections with these must be counted accordingly.

For $x, y \in \Sigma$ and $0 \leq s<F(x)$, define $g_{0}(s, x, y)$ to be 1 if the geodesic segment corresponding to the fiber $\mathcal{F}_{y}$ intersects the segment corresponding to the partial fiber

$$
\{(x, t): 0 \leq t<s\}
$$

and zero if not. Similarly, define $g_{1}(s, x, y)$ to be 1 if the geodesic segment corresponding to the fiber $\mathcal{F}_{y}$ intersects the segment corresponding to the partial fiber

$$
\{(x, t): s \leq t<F(x)\},
$$

and zero if not. If $\gamma$ is the geodesic ray whose initial tangent vector is $p \circ \pi(x, s)$, then the self-intersection count for the geodesic segment $\gamma[0, t]$ is given by

$$
\begin{aligned}
N(t ; \gamma)= & \frac{1}{2} \sum_{i=0}^{\tau} \sum_{j=1}^{\tau} h\left(\sigma^{i} x, \sigma^{j} x\right)-\sum_{i=1}^{\tau} g_{0}\left(s, x, \sigma^{i} x\right) \\
& -\sum_{i=0}^{\tau} g_{1}\left(S_{\tau+1} F(x)-t+s, \sigma^{\tau} x, \sigma^{i} x\right) \pm \text { error, }
\end{aligned}
$$

where

$$
\tau=\tau_{t}(x)=\min \left\{n \geq 0: S_{n+1} F(x) \geq t\right\} .
$$


The error term accounts for possible intersections between the initial and final segments, and hence is bounded in magnitude by 2 . Because it is bounded, it has no effect on the distribution of $\left(N(t)-\kappa t^{2}\right) / t$ in the large- $t$ limit. Note that whereas the first double sum in (28) will have magnitude $O\left(t^{2}\right)$ for large $t$, each of the single sums will have magnitude $O(t)$. Since the order of magnitude of the fluctuations in (2) is $O(T)$, it follows that the single sums in (28) will have an appreciable effect on the distribution of the normalized self-intersection counts in (2).

The following proposition summarizes the key features of the construction.

\section{PROPOSITION 3.7}

For any compact surface of constant curvature -1 there exists a topologically mixing shift $(\Sigma, \sigma)$ of finite type, a Hölder-continuous height function $F: \Sigma \rightarrow \mathbb{R}_{+}$, and functions $h_{m}: \Sigma \times \Sigma \rightarrow[0,1]$ satisfying (A1)-(A3) of Lemma 3.6 such that:

(SD-1) with only finitely many exceptions, each prime closed geodesic corresponds uniquely to a necklace;

(SD-2) the length of each such closed geodesic is $S_{n} F(x)$, where $n$ is the minimal period of the necklace $x$; and

(SD-3) the number of self-intersections of any such closed geodesic is given by (25), with $h$ defined by (27).

(Here a necklace is an equivalence class of periodic sequences, where two periodic sequences are considered equivalent if one is a shift of the other.) Furthermore, there exists a semiconjugacy of the symbolic flow $\phi_{t}$ on $\Sigma_{F}$ with the geodesic flow that is one-to-one except on a set of first category. For any geodesic $\gamma$, the number $N(t ; \gamma)$ of self-intersections of the segment $\gamma[0, t]$ is given by (28), with the error bounded by 2.

\section{Remark 3.8}

The choice of the Poincaré section in the construction of the suspension flow is important because it determines the locations of the discontinuities of the function $h$ in the representation (25), which in turn determine how well $h(x, y)$ can be represented by functions that depend on only finitely many coordinates of $x$ and $y$ (see property (A3) in Lemma 3.6). This choice is somewhat arbitrary; other Poincaré sections can be obtained in a number of ways, the simplest of which is by moving points of the original section forward a distance $r$ along the flow lines (equivalently, replacing the semiconjugacy $\pi$ of Proposition 3.1 by $\pi_{r}=\pi \circ \phi_{r}$ ). This has the effect of changing the function $h$ as follows. Define $h^{r}: \Sigma \times \Sigma \rightarrow\{0,1\}$ by setting $h^{r}(x, y)=1$ if the $(p \circ \pi)$-projections of the suspension flow segments

$$
\mathcal{F}_{x}^{r}:=\left\{\phi_{s}(x)\right\}_{r \leq s<F(x)+r} \quad \text { and } \quad \mathcal{F}_{y}^{r}:=\left\{\phi_{s}(y)\right\}_{r \leq s<F(y)+r}
$$

intersect transversally on $\Upsilon$ and by setting $h^{r}(x, y)=0$ if not. Clearly, the representation (25) for the self-intersection counts of closed geodesics remains valid with $h$ 
replaced by any $h^{r}$. Similarly, the representation (2) for the self-intersection count of an arbitrary geodesic segment will hold when $h, g_{0}$, and $g_{1}$ are replaced by $h^{r}$, $g_{0}^{r}$, and $g_{1}^{r}$, where $g_{i}^{r}$ are the obvious modifications of $g_{i}$. The function $h^{r}$ can be decomposed as

$$
h^{r}=\sum_{m=0}^{\infty} h_{m}^{r}+h_{\infty}^{r},
$$

where $h_{m}^{r}(x, y)$ and $h_{\infty}^{r}(x, y)$ are defined in analogous fashion to the functions $h_{m}$ and $h_{\infty}$ above, in particular, $h_{m}^{r}(x, y)=1$ if $m$ is the smallest positive integer such that $h^{r}\left(x^{\prime}, y^{\prime}\right)=1$ for all pairs $x^{\prime}, y^{\prime}$ that agree with $x, y$ in coordinates $|j| \leq m$, and $h_{m}^{r}(x, y)=0$ otherwise. These functions once again satisfy properties (A1)-(A2) of Lemma 3.6. Property (A3) is replaced by the following (A3)': if $\sum_{n \geq m} h_{n}^{\varepsilon}(x, y)+$ $h_{\infty}^{\varepsilon}(x, y) \neq 0$, then the geodesic segments corresponding to the suspension flow segments $\mathcal{F}_{x}^{r}$ and $\mathcal{F}_{y}^{r}$ intersect either

(a) at an angle less than $C \varrho^{m}$, or

(b) at a point at distance less than $C \varrho^{m}$ from one of the endpoints of $p \circ \pi\left(\mathcal{F}_{x}^{r}\right)$ or $p \circ \pi\left(\mathcal{F}_{y}^{r}\right)$.

\section{Gibbs states and thermodynamic formalism}

\subsection{Standing conventions}

We will adhere (mostly) to the notation and terminology of Bowen in [11]. However, we will suppress the dependence of various objects on the transition matrix $A$ of the underlying subshift of finite type, since this will be fixed throughout the paper: thus, the spaces of one-sided and two-sided sequences will be denoted by $\Sigma^{+}$ and $\Sigma$, respectively, and the spaces of $\alpha$-Hölder-continuous real-valued functions on these sequence spaces by $\mathcal{F}^{+}$and $\mathcal{F}$. (With one exception (Section 4.2), the Hölder exponent $\alpha$ will also be fixed throughout this paper, so henceforth we will refer to $\alpha$-Hölder functions as Hölder functions.) The spaces $\mathcal{F}=\mathscr{F}_{\alpha}$ and $\mathscr{F}^{+}=\mathscr{F}_{\alpha}^{+}$are Banach spaces with norm

$$
\begin{aligned}
& \|f\|=\|f\|_{\alpha}=|f|_{\alpha}+\|f\|_{\infty}, \quad \text { where } \\
& \qquad|f|_{\alpha}=\sup _{n \geq 0 x, y: x_{j}=y_{j} \forall|j| \leq n}|f(x)-f(y)| / \alpha^{n} .
\end{aligned}
$$

For any sequence $x \in \Sigma$ or $x \in \Sigma^{+}$and any interval $J=\{k, k+1, \ldots, l\}$ of $\mathbb{Z}$ or $\mathbb{N}$, denote by $x_{J}$ or $x(J)$ the subsequence $x_{k} x_{k+1} \cdots x_{l}$, and let $\Sigma_{J}(x)$ (or $\Sigma_{J}^{+}(x)$ ) be the cylinder set consisting of all sequences $y \in \Sigma$ such that $y(J)=x(J)$. For any $n \in \mathbb{N}$, let $[n]=\{1,2, \ldots, n\}$. For any continuous, real-valued function $\varphi$ and any probability measure $\lambda$ on $\Sigma$ or $\Sigma^{+}$, denote by $E_{\lambda} \varphi=\int \varphi d \lambda$ the expectation of 
$\varphi$ with respect to $\lambda$, by $\operatorname{Pr}(\varphi)$ the topological pressure of $\varphi$ (see [11, Lemma 1.20, Section $2 \mathrm{C}]$ ), and for each interval $J \subset \mathbb{Z}$, write

$$
S_{J} \varphi=\sum_{i \in J} \varphi \circ \sigma^{i}
$$

Following Bowen we will also write $S_{n} \varphi=S_{[n]} \varphi \circ \sigma^{-1}=\sum_{i=0}^{n-1} \varphi \circ \sigma^{i}$ for any integer $n \geq 1$.

\subsection{Gibbs states}

For each real-valued function $\varphi \in \mathcal{F}$ there is a unique Gibbs state $\mu_{\varphi}$, which is by definition a shift-invariant probability measure $\mu_{\varphi}$ on $\Sigma$ for which there are constants $0<C_{1}<C_{2}<\infty$ such that, for every finite interval $J \subset \mathbb{Z}$,

$$
C_{1} \leq \frac{\mu_{\varphi}\left(\Sigma_{J}(x)\right)}{\exp \left\{S_{J} \varphi(x)-|J| \operatorname{Pr}(\varphi)\right\}} \leq C_{2}
$$

for all $x \in \Sigma$. When the potential function $\varphi$ is fixed, we will delete the subscript $\varphi$ and write $\mu$ in place of $\mu_{\varphi}$.

If the underlying shift $(\Sigma, \sigma)$ is topologically mixing-as we will assume throughout - then every Gibbs state $\mu=\mu_{\varphi}$ is mixing (and therefore ergodic) and has positive entropy. Moreover, there exists $\alpha=\alpha_{\varphi}<1$ such that for every $n \geq 1$, all cylinder sets $\Sigma_{[0, n]}(x)$ of generation $n$ have $\mu$-probabilities less than $\alpha^{n}$. In addition, correlations decay exponentially, in the following sense. For any subset $J \subset \mathbb{Z}$, let $\mathscr{B}_{J}$ be the $\sigma$-algebra of Borel subsets $G$ of $\Sigma$ whose indicator functions $\mathbf{1}_{G}$ depend only on the coordinates $n \in J$. Then for each Gibbs state $\mu=\mu_{\varphi}$, there exist constants $C=C_{\varphi}<\infty$ and $0<\beta=\beta_{\varphi}<1$ such that for each $n \geq 1$,

$$
\begin{aligned}
& \left|\mu\left(G \cap G^{\prime}\right)-\mu(G) \mu\left(G^{\prime}\right)\right| \\
& \quad \leq C \beta^{n} \mu(G) \mu\left(G^{\prime}\right) \quad \forall G \in \mathcal{B}_{(-\infty, 0]}, G^{\prime} \in \mathcal{B}_{[n, \infty)} .
\end{aligned}
$$

This implies that, for any specification of the "past" $\cdots \omega_{-1} \omega_{0}$, the conditional distribution of the "future" $\omega_{n} \omega_{n+1} \cdots$ differs from the unconditional distribution by at most $2 C \beta^{n}$ in total variation norm. The exponential mixing property can be expressed in the following equivalent form (see [31, pp. 29-30]): for any two $\alpha$-Hölder functions $v, w$ such that $E_{\mu} w=0$,

$$
\left|E_{\mu} v\left(w \circ \sigma^{n}\right)\right| \leq C \beta^{n}\|v\|_{\infty}\|w\|_{\alpha},
$$

where $\|w\|_{\alpha}$ is the Hölder norm of $w$.

The uniform mixing property (33) implies that it is unlikely that a random sequence $x \in \Sigma$ chosen according to the law of a Gibbs state will have long repeating 
strings at fixed locations. This is made precise in the next lemma; it will be used in Section 7 below (see Lemma 7.7) to show that self-intersections at very small angles are highly unlikely.

\section{LEMMA 4.1}

For any Gibbs state $\mu$ there exists $0<\beta<1$ such that, for all $k \neq 0$ and all sufficiently large $m \geq 1$,

$$
\mu\left\{x \in \Sigma: x_{i}=x_{i+k} \text { for all } 0 \leq i \leq m\right\} \leq \beta^{m} .
$$

\section{Proof}

The mixing inequality (33) and $\sigma$-invariance of $\mu$ imply that there exist an integer $L \geq 1$ and $0<\beta<1$ such that, for every $L^{\prime} \geq L$ and every symbol $a \in \mathcal{A}$ of the underlying alphabet,

$$
\mu\left(x_{L^{\prime}+n}=a \mid \mathscr{B}_{(-\infty, n]}\right) \leq \beta \quad \text { for all } n \in \mathbb{Z} .
$$

We will consider two separate cases: first, $k \geq L$; and second, $1 \leq k<L$. (Since every Gibbs state is $\sigma$-invariant, it suffices to consider only positive values of $k$.) In the first case,

$$
\begin{aligned}
\mu\left(x_{i}=x_{k+i} \forall 1 \leq i \leq n L\right) & \leq \mu\left(x_{i L}=x_{k+i L} \forall 1 \leq i \leq n\right) \\
& =E_{\mu} \prod_{i=1}^{n} \mu\left(x_{i L}=x_{k+i L} \mid \mathscr{B}_{(-\infty, k+(i-1) L]}\right) \\
& \leq \beta^{n} .
\end{aligned}
$$

In the case $1 \leq k<L$, there will be a multiple of $k$ in every interval of length $L$, so we can choose $m_{1}<m_{2}<\cdots<m_{n}$ such that $j L \leq m_{j} k<j L+L$ for each $j \leq n$. Now the requirement that $x_{i}=x_{i+k}$ for all $1 \leq i \leq n L$ forces $x_{0}=x_{m_{j} k}$ for every $j \leq n$; consequently,

$$
\begin{aligned}
\mu\left(x_{i}=x_{k+i} \forall 1 \leq i \leq n L\right) & \leq \mu\left(x_{0}=x_{m_{j} k} \forall 1 \leq j \leq n\right) \\
& =E_{\mu} \prod_{j=1}^{[n / 2]} \mu\left(x_{m_{2 j} k}=x_{0} \mid \mathcal{B}_{\left(-\infty, m_{2 j-2} k\right]}\right) \\
& \leq \beta^{[n / 2]} .
\end{aligned}
$$

Two functions $\varphi, \psi \in \mathscr{F}$ are said to be cohomologous if their difference is a cocycle $u-u \circ \sigma$, with $u \in \mathscr{F}$. If $\varphi$ and $\psi$ are cohomologous, then $\mu_{\varphi}=\mu_{\psi}$ and 
$\operatorname{Pr}(\varphi)=\operatorname{Pr}(\psi)$. According to a theorem of Livsic [11, Lemma 1.6], for every $\alpha$ Hölder function $\varphi$ there exist $\sqrt{\alpha}$-Hölder functions $\varphi^{+}, \varphi^{-}$both cohomologous to $\varphi$ (and therefore mutually cohomologous) such that $\varphi^{+}(x)$ depends only on the forward coordinates $x_{1}, x_{2}, \ldots$ of $x$ and $\varphi^{-}(x)$ depends only on the backward coordinates $x_{0}, x_{-1}, \ldots$.

For any function $\varphi \in \mathcal{F}^{+}$, the Gibbs state $\mu_{\varphi}$ is related to the Perron-Frobenius eigenfunction $h_{\varphi}$ and eigenmeasure $v_{\varphi}$ of the Ruelle operator $\mathscr{L}_{\varphi}: \mathscr{F}^{+} \rightarrow \mathcal{F}^{+}$associated with $\varphi$ (see [11, Chapter 1 , Section C]). In particular, if $h_{\varphi}$ and $v_{\varphi}$ are normalized so that $v_{\varphi}$ and $h_{\varphi} v_{\varphi}$ both have total mass 1 , and if $\lambda_{\varphi}$ is the Perron-Frobenius eigenvalue, then

$$
d \mu_{\varphi}=h_{\varphi} d \nu_{\varphi} \quad \text { and } \quad \lambda_{\varphi}=\exp \{\operatorname{Pr}(\varphi)\}
$$

\subsection{Suspensions}

Say that a function $f \in \mathcal{F}$ (or $\mathcal{F}^{+}$) is nonarithmetic if there is no function $g \in \mathcal{F}$ valued in a discrete additive subgroup of $\mathbb{R}$ to which $f$ is cohomologous. If $F \in \mathscr{F}$ is strictly positive, then the suspension flow with height function $F$ is topologically mixing if and only if $F$ is nonarithmetic (see, e.g., [30]). This is the case, in particular, for the suspension flow discussed in Section 3.

Assume henceforth that $F$ is a strictly positive, nonarithmetic, Hölder-continuous function on $\Sigma$, and let $\Sigma_{F}$ be the corresponding suspension space. For each $\sigma$ invariant probability measure $\mu$ on $\Sigma$, define the suspension of $\mu$ relative to $F$ to be the flow-invariant probability measure $\mu^{*}$ on $\Sigma_{F}$ with cylinder probabilities

$$
\mu^{*}\left(\Sigma_{[n]}(x) \times[0, a]\right)=\frac{a \mu\left(\Sigma_{[n]}(x)\right)}{\int_{\Sigma} F d \mu}
$$

for any cylinder set $\Sigma_{[n]}(x)$ and any $a \geq 0$ such that $a \leq F$ on $\Sigma_{[n]}(x)$. (Here and elsewhere we use the notation $[n]$ to denote the set of integers $\{1,2, \ldots, n\}$.) For the geodesic flow on a compact, negatively curved surface, both the Liouville measure and the maximum entropy measure lift to the suspensions of Gibbs states; for the maximum entropy measure, the corresponding Gibbs state is $\mu_{-\theta F}$ where $\theta>0$ is the unique real number such that $\operatorname{Pr}(-\theta F)=0$, and this value of $\theta$ is the topological entropy of the flow (see [1], also [22]). If the surface has constant negative curvature, then the Liouville and maximum entropy measures are the same, but if the surface has variable negative curvature, then the Liouville measure is mutually singular with the maximum entropy measure, and so the potential function for the corresponding Gibbs state is not cohomologous to $-\delta F$. This is what accounts for the difference between constant and variable negative curvature in Theorem 1.3.

If the suspension flow is topologically mixing, then the suspension of any Gibbs state is mixing for the flow. This fact is equivalent to a renewal theorem, which can 
be formulated as follows. For each $T \in \mathbb{R}_{+}$and each $x \in \Sigma$, define

$$
\begin{aligned}
\tau(x) & =\tau_{T}(x)=\min \left\{n \geq 1: S_{n} F(x)>T\right\} \quad \text { and } \\
R_{T}(x) & =S_{\tau(x)} F(x)-T .
\end{aligned}
$$

PROPOSITION 4.2

Assume that the shift $(\Sigma, \sigma)$ is topologically mixing and that $F \in \mathcal{F}$ is positive and nonarithmetic. Then for any Gibbs state $\mu$ and all bounded, continuous functions $f, g: \Sigma \rightarrow \mathbb{R}$ and $h: \mathbb{R} \rightarrow \mathbb{R}$,

$$
\begin{aligned}
& \lim _{T \rightarrow \infty} \int_{\Sigma} f(x) g\left(\sigma^{\tau(x)}(x)\right) h\left(R_{T}(x)\right) d \mu(x) \\
& =\int f(x) d \mu(x) \times \int_{\Sigma_{F}} g(x) h(t) d \mu^{*}(x, t),
\end{aligned}
$$

where $\mu^{*}$ is the suspension of $\mu$.

The special case where $f \equiv g \equiv 1$ is of particular interest, because it yields estimates of the probability that $R_{T}$ falls in an interval. In particular, it implies that there exists $C<\infty$ such that, for all $\varepsilon>0$, all $a \geq 0$, and all sufficiently large $T$ (i.e., all $T>t_{\varepsilon}$ ),

$$
\mu\left\{x \in \Sigma: a \leq R_{T}(x) \leq a+\varepsilon\right\} \leq C \varepsilon
$$

We will say that two (or more) weakly convergent sequences $X_{T}, Y_{T}$ of random variables, vectors, or sequences are asymptotically independent as $T \rightarrow \infty$ if the joint distribution of $\left(X_{T}, Y_{T}\right)$ converges weakly to the product of the limit distributions of $X_{T}$ and $Y_{T}$, that is, if for all bounded, continuous real-valued functions $u, v$,

$$
\lim _{T \rightarrow \infty} E u\left(X_{T}\right) v\left(Y_{T}\right)=\left(\lim _{T \rightarrow \infty} E u\left(X_{T}\right)\right)\left(\lim _{T \rightarrow \infty} E v\left(Y_{T}\right)\right)
$$

In this terminology, Proposition 4.2 asserts that the "overshoot" random variable $R_{T}(x)$ is asymptotically independent of the state variables $x$ and $\sigma^{\tau(x)}(x)$.

If $x \in \Sigma$ is chosen randomly according to an ergodic, shift-invariant probability measure $\mu$, then $\tau_{T}(x)$ will be random. However, when $T$ is large the random variable is to first order of approximation "predictable" in that the error in the approximation $\tau_{T} \approx T / E_{\mu} F$ is of order $O_{P}(1 / \sqrt{T})$. More precisely, we have the following.

\section{PROPOSITION 4.3}

Assume that $F: \Sigma \rightarrow \mathbb{R}$ and $g: \Sigma \rightarrow \mathbb{R}^{k}$ are Hölder-continuous functions, with $F>0$, and let $\mu$ be a Gibbs state for the shift $(\Sigma, \sigma)$. Then there exist a constant 
$b>0$ (depending on $\mu$ and $F$ ) and $a k \times k$ positive semidefinite matrix $\mathbf{M}$ (depending on $\mu, F$, and $g$ ) such that as $T \rightarrow \infty$,

$$
\begin{aligned}
\frac{\tau_{T}-T / E_{\mu} F}{b \sqrt{T}} & \Longrightarrow \operatorname{Normal}(0,1) \quad \text { and } \\
\frac{S_{\tau_{T}} g-T E_{\mu} g / E_{\mu} F}{\sqrt{T}} & \Longrightarrow \operatorname{Normal}_{k}(\mathbf{0}, \mathbf{M}) .
\end{aligned}
$$

Moreover, the limiting covariance matrix $\mathbf{M}$ is strictly positive definite unless some linear combination of the coordinate functions $g_{i}$ is cohomologous to $F+c$ for some constant c. Finally, the random vector $\left(S_{\tau} g-T E_{\mu} g / E_{\mu} F\right) / T^{1 / 2}$ and the random variable $\left(\tau_{T}-T / E_{\mu} F\right) / T^{1 / 2}$ are asymptotically independent of the overshoot $R_{T}(x)=S_{\tau(x)} F(x)-T$ and the state variables $x$ and $\sigma^{\tau(x)} x$.

Both (41) and (42) are consequences of Ratner's central limit theorem. (See in particular [34, proof of Theorem 2.1]. The vector-valued central limit theorem follows from the scalar central limit theorem by the so-called Cramer-Wold devicesee, e.g., [4, Chapter 1].) The last assertion (regarding asymptotic independence) can be proved by standard methods in renewal theory (see, e.g., [39]); roughly speaking, it holds because the values of the random variables $R_{T}(x)$ and $\sigma^{\tau(x)} x$ are mainly determined by the last $O(1)$ steps before time $\tau(x)$, whereas the values of $\left(S_{\tau} g-\right.$ $\left.T^{2} E_{\mu} g /\left(E_{\mu} F\right)^{2}\right) / T^{3 / 2}$ and other "bulk" random variables are mostly determined long before time $\tau(x)$.

\section{5. $U$-statistics}

\subsection{U -statistics with random limits of summation}

Let $(\Sigma, \sigma)$ be a two-sided shift of finite type, and let $F: \Sigma \rightarrow(0, \infty)$ be a Höldercontinuous function. Assume that $F$ is nonarithmetic: this ensures that the conclusions of Propositions 4.2 and 4.3 are valid. As in Section 4.3, define $\tau=\tau_{T}: \Sigma \rightarrow \mathbb{Z}_{+}$ to be the first passage time to the level $T>0$ by the sequence $S_{n} F$ (see equation (38)). Let $h: \Sigma \times \Sigma \rightarrow \mathbb{R}$ be a symmetric, Borel-measurable function. Our interest in this section is the distribution of the random variable

$$
U_{T}(x):=\sum_{i=1}^{\tau(x)} \sum_{j=1}^{\tau(x)} h\left(\sigma^{i} x, \sigma^{j} x\right) \quad \text { for } x \in \Sigma,
$$

under a Gibbs state $\mu$ or, more generally, under a probability measure that is absolutely continuous with respect to a Gibbs state. Random variables of this form-but with the random time $\tau(x)$ replaced by a constant $n$-are known in probability theory as $U$-statistics, and have a well-developed limit theory (see [19], [16]). Unfortunately, 
the standard results of this literature do not apply here, for three reasons: (a) because here the limits of summation in (43) are themselves random variables, (b) because no continuity requirements have been imposed on the function $h$, and (c) because of the peculiar nature of the dependence in the sequence $\left\{\sigma^{n} x\right\}_{n \in \mathbb{Z}}$.

\subsection{Convergence in law under Gibbs states}

Fix a probability measure $\lambda$ on $\Sigma$.

\section{HYPOTHESIS 5.1}

The kernel $h$ admits a decomposition $h=\sum_{m=1}^{\infty} h_{m}$ such that

(H0) each $h_{m}: \Sigma \rightarrow \mathbb{R}$ is a symmetric function of its arguments;

(H1) there exists $C<\infty$ such that $\sum_{m \geq 1}\left|h_{m}\right| \leq C$ on $\Sigma \times \Sigma$;

(H2) $\quad h_{m}(x, y)$ depends only on the coordinates $x_{j}, y_{j}$ such that $|j| \leq m$; and

(H3) there exist $C<\infty$ and $0<\beta<1$ such that for all $m \geq 1$ and $j \in \mathbb{Z}$,

$$
\int_{\Sigma}\left|h_{m}\left(x, \sigma^{j} x\right)\right| d \lambda(x) \leq C \beta^{m}
$$

\section{Definition 5.2}

For any bounded, symmetric, measurable function $h: \Sigma \times \Sigma \rightarrow \mathbb{R}$ and any Borel probability measure $\lambda$ on $\Sigma$, define the Hoeffding projection $h_{+}: \Sigma \rightarrow \mathbb{R}$ of $u$ relative to $\lambda$ by

$$
h_{+}(x)=\int_{\Sigma} h(x, y) d \lambda(y) .
$$

Say that the kernel $h$ is centered relative to $\lambda$ if its Hoeffding projection is identically zero.

Our interest is in the large- $T$ limiting behavior of the random variable $U_{T}$ defined by (43) (more precisely, its distribution) under a Gibbs state or a probability measure that is absolutely continuous with respect to a Gibbs state. Observe that if $\mu$ is a Gibbs state and if $h$ satisfies Hypothesis 5.1 relative to $\mu$, then the corresponding Hoeffding projection $h_{+}(x)$ is Hölder-continuous on $\Sigma$, even though $h$ itself might not be continuous. The following theorem will show that under Hypothesis 5.1, two types of limit behavior are possible, depending on whether or not $h_{+}$is cohomologous to a scalar multiple $a F$ of $F$. Set

$$
\tilde{\tau}_{T}=\frac{\tau-T / E_{\mu} F}{\sqrt{T}} .
$$

\section{THEOREM 5.3}

Let $\mu=\mu_{\varphi}$ be a Gibbs state, and let $h: \Sigma \times \Sigma \rightarrow \mathbb{R}$ be a function that satisfies 
Hypothesis 5.1, for $\lambda=\mu$. If the Hoeffding projection $h_{+}$of $h$ relative to $\mu$ is cohomologous to a $F$ for some scalar $a \in \mathbb{R}$, then as $T \rightarrow \infty$,

$$
\tilde{U}_{T}=\frac{U_{T}-\left(a / E_{\mu} F\right) T^{2}}{T} \stackrel{D}{\longrightarrow} G
$$

for some probability distribution $G$ on $\mathbb{R}$. Otherwise,

$$
\tilde{U}_{T}=\frac{U_{T}-\left(E_{\mu} h_{+} / E_{\mu} F^{2}\right) T^{2}}{T^{3 / 2}} \stackrel{D}{\longrightarrow} \text { Gaussian }
$$

for a proper Gaussian distribution on $\mathbb{R}$. Furthermore, in either case the random vector $\left(\tilde{U}_{T}, \tilde{\tau}_{T}\right)$, the state variables $x, \sigma^{\tau(x)}$, and the overshoot random variable $R_{T}$ are asymptotically independent as $T \rightarrow \infty$.

\section{Proof strategy}

The logic of the proof is as follows. First, we will show that the theorem is true for centered kernels $h$ such that $h(x, y)$ depends only on finitely many coordinates of the arguments $x, y$. This will use Proposition 4.3. Second, we will prove by an approximation argument that the truth of the theorem for centered kernels can be deduced from the special case of centered kernels that depend on only finitely many coordinates. This step will use moment estimates that depend on Hypothesis 5.1 (in particular, on the critical assumption (H3)). Third, we will show that to prove the theorem in the general case it suffices to consider the case where the kernel $h$ is centered. For ease of exposition, we will present the third step before the second; however, this step will rely on the other two.

Observe that the validity of (46) and (47) is not affected by rescaling of either $T$ or $h$. Consequently, there is no loss of generality in assuming that $E_{\mu} F=1$ and that the constants $C, C^{\prime}$ in Hypothesis 5.1 are $C=C^{\prime}=1$.

\section{Step 1}

Assume first that $h$ is centered (thus, $h_{+}$is cohomologous to $a F$ with $a=0$, and so case (46) of Theorem 5.3 applies) and that $h(x, y)$ depends only on the coordinates $x_{1} x_{2} \cdots x_{m}$ and $y_{1} y_{2} \cdots y_{m}$. Then the function $h$ assumes only finitely many different values, and these are given by a symmetric, real, square matrix $h(\xi, \zeta)$, where $\xi$ and $\zeta$ range over the set $\Sigma_{m}$ of all length- $m$ words occurring in infinite sequences $x \in \Sigma$. This matrix induces a real, Hermitian operator $L$ on the finite-dimensional subspace of $L^{2}(\Sigma, \mu)$ consisting of functions that depend only on the coordinates $x_{1} x_{2} \cdots x_{m}$. Let $D_{m}$ be the dimension of this subspace. Because $h$ is centered, the operator $L$ contains the constants in its null space. Consequently, all other eigenfunctions $\varphi_{k}$ are orthogonal to the constant function 1, and thus, in particular, have mean zero. It follows by the spectral theorem for symmetric matrices that the $U$-statistic (43) can 
be written as

$$
U_{T}(x)=\sum_{i=1}^{\tau(x)} \sum_{j=1}^{\tau(x)} \sum_{k=2}^{D_{m}} \lambda_{k} \varphi_{k}\left(\sigma^{i} x\right) \varphi_{k}\left(\sigma^{j} x\right)=\sum_{k=2}^{D_{m}} \lambda_{k}\left(\sum_{i=1}^{\tau(x)} \varphi_{k}\left(\sigma^{i} x\right)\right)^{2} .
$$

Therefore, Proposition 4.3 implies that as $T \rightarrow \infty$,

$$
\left(\tilde{\tau}_{T},\left(\frac{1}{\sqrt{T}} \sum_{i=1}^{\tau} \varphi_{k} \circ \sigma^{i}\right)_{2 \leq k \leq D(m)}\right) \stackrel{D}{\longrightarrow} \mathrm{N}(\mathbf{0}, \mathbf{A})
$$

for some possibly degenerate $\left(D_{m}-1\right)$-dimensional multivariate normal distribution $N(0, A)$. The convergence (46) follows, with $a=0$ and $G$ the distribution of the quadratic form of the multivariate normal. Proposition 4.3 also implies that the random vector (48) is asymptotically independent of $x, \sigma^{\tau(x)} x$, and $R_{T}(x)$; consequently, so is the random vector with components $U_{T} / T$ and $\tilde{\tau}_{T}$.

\section{Step 3}

Assume that the result is true for all centered kernels. We will show that the theorem then holds for any noncentered kernel satisfying Hypothesis 5.1. Recall that if $h$ satisfies Hypothesis 5.1, then its Hoeffding projection $h_{+}$is Hölder-continuous. There are two cases to consider, according to whether or not $h_{+}$is cohomologous to a scalar multiple of $F$. Consider first the case where $h_{+}$is cohomologous to $a F$ for some $a \in \mathbb{R}$. Thus, $E_{\mu} h_{+}=a$ (since $E_{\mu} F=1$ ), and so for some coboundary $w-w \circ \sigma$,

$$
\begin{aligned}
h(x, y) & =h_{0}(x, y)+h_{+}(x)+h_{+}(y)-a \\
& =h_{0}(x, y)+a F(x)+a F(y)+w(x)-w(\sigma x)-w(y)-w(\sigma y)-a,
\end{aligned}
$$

where $h_{0}(x, y)$ is centered. This implies that

$$
\begin{aligned}
U_{T}= & \sum_{i=1}^{\tau(x)} \sum_{j=1}^{\tau(x)} h\left(\sigma^{i} x, \sigma^{j} x\right) \\
= & \sum_{i=1}^{\tau(x)} \sum_{j=1}^{\tau(x)} h_{0}\left(\sigma^{i} x, \sigma^{j} x\right)+2 a \tau(x) S_{\tau(x)} F(x)-a \tau(x)^{2} \\
& +\tau(x)\left(w(x)-w\left(\sigma^{\tau(x)} x\right)\right) \\
= & \sum_{i=1}^{\tau(x)} \sum_{j=1}^{\tau(x)} h_{0}\left(\sigma^{i} x, \sigma^{j} x\right)+a T^{2}+a(\tau(x)-T)^{2}+a \tau(x) R_{T}(x) \\
& +\tau(x)\left(w(x)-w\left(\sigma^{\tau(x)} x\right)\right) \\
= & : V_{T}+a T^{2}+a(\tau(x)-T)^{2}+a \tau(x) R_{T}(x)+\tau(x)\left(w(x)-w\left(\sigma^{\tau(x)} x\right)\right),
\end{aligned}
$$


where $V_{T}$ is the $U$-statistic (43) with the kernel $h$ replaced by the centered kernel $h_{0}$. Now as $T \rightarrow \infty, \tau(x) / T \rightarrow 1$ almost surely, by the ergodic theorem, and both $R_{T}$ and $\tilde{\tau}_{T}$ converge in distribution, by Propositions 4.2 and 4.3. Consequently, the convergence (46) and the joint asymptotic independence assertions hold because by assumption they hold for centered kernels.

Next, consider the case where $h_{+}$is not cohomologous to a scalar multiple of $F$; we must prove (47). As above, we assume without loss of generality that $E_{\mu} F=1$. Let $h_{0}$ be the centered kernel defined by

$$
h(x, y)=h_{0}(x, y)+h_{+}(x)+h_{+}(y)-b,
$$

where $b=E_{\mu} h_{+}$; then

$$
\begin{aligned}
U_{T}= & \sum_{i=1}^{\tau(x)} \sum_{j=1}^{\tau(x)} h_{0}\left(\sigma^{i} x, \sigma^{j} x\right)+2 \tau(x) S_{\tau(x)} h_{+}(x)-b \tau(x)^{2} \\
= & \sum_{i=1}^{\tau(x)} \sum_{j=1}^{\tau(x)} h_{0}\left(\sigma^{i} x, \sigma^{j} x\right)+2 \tau(x)\left(S_{\tau(x)} h_{+}(x)-b \tau(x)\right)+b T^{2} \\
& +b\left(\tau(x)^{2}-T^{2}\right) .
\end{aligned}
$$

Now consider the effect of dividing this quantity by $T^{3 / 2}$. Since the kernel $h_{0}$ is centered, the double sum divided by $T$ converges in distribution (by our hypothesis that the theorem is true for centered kernels); hence, if it is divided by $T^{3 / 2}$, it will converge to zero. Thus, asymptotically as $T \rightarrow \infty$, the distribution of $\left(U_{T-b T^{2}}\right) / T^{3 / 2}$ is determined by the remaining terms $2 \tau\left(S_{\tau}-b \tau\right) / T^{3 / 2}$ and $b\left(\tau^{2}-T^{2}\right) / T^{3 / 2}$. The ergodic theorem implies that $\tau / T \rightarrow 1$, and the central limit theorem (Proposition 4.3) implies that $\left(S_{\tau} h_{+}-b T\right) / T^{1 / 2}$ and $\tilde{\tau}_{T}=(\tau-T) / T^{1 / 2}$ converge jointly in distribution to a 2-dimensional Gaussian distribution; consequently,

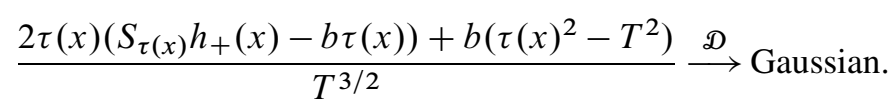

This proves (47). The asymptotic independence assertions follow directly from Proposition 4.3 .

Step 2

Assume, finally, that $h$ is a centered kernel which satisfies Hypothesis 5.1. Without loss of generality, we can assume that the functions $h_{m}$ in the decomposition $h=$ $\sum_{m=1}^{\infty} h_{m}$ are themselves centered, because replacing each $h_{m}(x, y)$ by $h_{m}(x, y)-$ 
$h_{m}^{+}(x)-h_{m}^{+}(y)$ does not change the validity of Hypothesis 5.1. Set

$$
v_{m}=\sum_{k=1}^{m} h_{k} \quad \text { and } \quad w_{m}=\sum_{k=m+1}^{\infty} h_{k} .
$$

Then each $v_{m}$ is centered, so by Step 1, the result is true if $v_{m}$ is substituted for $h$ in the definition of $U_{T}$ (but of course the limit distribution $G=G_{m}$ will depend on $m$ ). Consequently, to prove the result for $h$ it suffices to show that for any $\varepsilon>0$ there exists $m$ sufficiently large so that

$$
\mu\left\{x:\left|\sum_{i=1}^{\tau(x)} \sum_{j=1}^{\tau(x)} w_{m}\left(\sigma^{i} x, \sigma^{j} x\right)\right|>\varepsilon T\right\}<\varepsilon
$$

for all sufficiently large $T$.

Fix $0<\delta<1 / 6$, and set

$$
\begin{aligned}
& n_{-}=n_{-}(T)=\left\lfloor T-T^{1 / 2+\delta}\right\rfloor \quad \text { and } \\
& n_{+}=n_{+}(T)=\left\lfloor T+T^{1 / 2+\delta}\right\rfloor .
\end{aligned}
$$

By the central limit theorem (Proposition 4.3), $n_{-}<\tau<n_{+}$with $\mu$-probability approaching 1 as $T \rightarrow \infty$; thus $\tau(x)$ is essentially limited to one of $T^{1 / 2+\delta}$ different possible values. Therefore, by the Chebyshev inequality and a crude union bound, to establish (49) it suffices to prove the following.

\section{LEMMA 5.4}

For each $\varepsilon>0$ there exists $m$ sufficiently large that for all large $T$,

$$
\begin{aligned}
& E_{\mu}\left(\sum_{i=1}^{n_{-}} \sum_{j=1}^{n_{-}} w_{m}\left(\sigma^{i} x, \sigma^{j} x\right)\right)^{2}<\varepsilon T^{2} \text { and } \\
& \max _{n_{-} \leq n \leq n_{+}} E_{\mu}\left(\sum_{i=1}^{n} \sum_{j=1}^{n} w_{m}\left(\sigma^{i} x, \sigma^{j} x\right)-\sum_{i=1}^{n_{-}} \sum_{j=1}^{n_{-}} w_{m}\left(\sigma^{i} x, \sigma^{j} x\right)\right)^{4}<\varepsilon T^{3+2 \delta} .
\end{aligned}
$$

\section{Proof of (50)}

This will use assumption (H3) and also the fact that Gibbs states have exponentially decaying correlations (see (33)). Since $h_{k}(x, y)$ depends only on the coordinates $x_{i}, y_{i}$ with $|i| \leq k$, and since $\left|h_{k}\right| \leq 1$ (see the earlier remark on scaling), exponential correlation decay implies that for all $k, r \geq 1$,

$$
\left|E\left(h_{k}\left(x, \sigma^{k+r} x\right) \mid \mathscr{B}_{(-\infty, k] \cup[2 k+2 r, \infty)}\right)\right| \leq C \beta^{r} .
$$


For convenience, we will assume that the constants $0<\beta<1$ in (33) and in assumption (H3) are the same (if the two constants are different, replace the smaller with the larger).

When the square in (50) is expanded, the resulting terms have the form

$$
E h_{k}\left(\sigma^{i} x, \sigma^{j} x\right) h_{k^{\prime}}\left(\sigma^{i^{\prime}} x, \sigma^{j^{\prime}} x\right)
$$

with $k, k^{\prime} \geq m$ and $i, i^{\prime}, j, j^{\prime} \leq n_{-} \leq T$. Let $\Delta$ be the largest integer such that one of the four indices $i, i^{\prime}, j, j^{\prime}$ is separated from all of the other three by a gap of size $\Delta$, and let $k_{*}=\max \left(k, k^{\prime}\right)$. Then by the exponential correlation decay inequality (52) and assumption (H3) (using the fact that $\left|h_{k} h_{k^{\prime}}\right| \leq\left|h_{k_{*}}\right|$ ),

$$
\left|E h_{k}\left(\sigma^{i} x, \sigma^{j} x\right) h_{k^{\prime}}\left(\sigma^{i^{\prime}} x, \sigma^{j^{\prime}} x\right)\right| \leq C \min \left(\beta^{\Delta-2 k_{*}}, \beta^{k_{*}}\right) .
$$

For any given value of $\Delta \geq 1$, the number of quadruples $i, i^{\prime}, j, j^{\prime} \leq T$ with maximal gap size $\Delta$ is bounded above by $24 T^{2}(2 \Delta+1)^{2}$. (There are roughly $T^{2}$ choices for two of the indices; once such a choice $\left(l, l^{\prime}\right)$ is made, then one of the remaining indices must be located within the interval of radius $\Delta$ centered at $l$, and the other within the corresponding interval centered at $l^{\prime}$. The factor $24=4$ ! accounts for the possible permutations of the indices.) Furthermore, for each $k_{*} \geq m$ the number of pairs $k, k^{\prime} \geq m$ such that $\max \left(k, k^{\prime}\right)=k_{*}$ is less than $2 k_{*}$. Consequently,

$$
E_{\mu}\left(\sum_{i=1}^{n_{-}} \sum_{j=1}^{n_{-}} w_{m}\left(\sigma^{i} x, \sigma^{j} x\right)\right)^{2} \leq C^{\prime} T^{2} \sum_{k_{*}=m}^{\infty} \sum_{\Delta=0}^{\infty}(2 \Delta+1)^{2} k_{*} \min \left(\beta^{\Delta-2 k_{*}}, \beta^{k_{*}}\right),
$$

where $C^{\prime}=48$. By choosing $m$ sufficiently large one can make this bound smaller than $\varepsilon T^{2}$.

\section{Proof of (51)}

This is similar to the proof of (50), the difference being that here it is necessary to count octuples instead of quadruples. The key once again is the exponential correlation decay inequality (52): this implies that for any four triples $i_{r}, j_{r}, k_{r}$,

$$
\left|E_{\mu} \prod_{r=1}^{4} h_{k_{r}}\left(\sigma^{i_{r}} x, \sigma^{j_{r}} x\right)\right| \leq C \min \left(\beta^{\Delta-2 k_{*}}, \beta^{k_{*}}\right),
$$

where $k_{*}=\max _{1 \leq r \leq 4} k_{r}$ and where $\Delta$ is the maximal gap separating one of the indices $i_{r}, j_{r}$ from the remaining seven. For each $r \leq 4$, the indices $i_{r}, j_{r}$ that occur in (51) are constrained as follows (taking $i_{r}$ to be the smaller of the two): either

$$
1 \leq i_{r} \leq n_{-} \leq j_{r} \leq n \quad \text { or } \quad n_{-} \leq i_{r} \leq j_{r} \leq n
$$


Consequently, for each $\Delta \geq 1$, the total number of octuples $\left(i_{r}, j_{r}\right)_{1 \leq r \leq 4}$ with maximal gap $\Delta$ that occur when the fourth power in (51) is expanded is bounded above by $C^{\prime} \Delta^{3} T^{3+2 \delta}$ for some constant $C^{\prime}<\infty$ independent of $T$ and $\Delta$. For each $k_{*} \geq m$, the number of quadruples $k_{1}, k_{2}, k_{3}, k_{4}$ with maximum value $k_{*}$ is bounded above by $4 k_{*}^{3}$. Therefore, for each $n$ such that $n_{-} \leq n \leq n_{+}$,

$$
\begin{gathered}
E_{\mu}\left(\sum_{i=1}^{n} \sum_{j=1}^{n} w_{m}\left(\sigma^{i} x, \sigma^{j} x\right)-\sum_{i=1}^{n_{-}} \sum_{j=1}^{n_{-}} w_{m}\left(\sigma^{i} x, \sigma^{j} x\right)\right)^{4} \\
\leq C^{\prime \prime} T^{3+2 \delta} \sum_{k_{*}=m}^{\infty} \sum_{\Delta=0}^{\infty} \Delta^{3} k_{*}^{3} \min \left(\beta^{\Delta-2 k_{*}}, \beta^{k_{*}}\right)
\end{gathered}
$$

for a constant $C^{\prime \prime}<\infty$ independent of $T$ and $m$. By choosing $m$ large one can make this bound smaller than $\varepsilon T^{3+2 \delta}$.

\subsection{Extensions}

\section{COROLLARY 5.5}

Let $\mu=\mu_{\varphi}$ be a Gibbs state, and let $\lambda$ be a Borel probability measure on $\Sigma$ that is absolutely continuous with respect to $\mu$ and such that the likelihood ratio $d \lambda / d \mu$ is continuous on $\Sigma$. Let $h: \Sigma \times \Sigma \rightarrow \mathbb{R}$ be a function that satisfies Hypothesis 5.1 relative to $\mu$. Then all of the conclusions of Theorem 5.3 remain valid under the measure $\lambda$, and the joint limit distribution of $\tilde{U}_{T}, \tilde{\tau}_{T}$, and $R_{T}$ is the same under $\lambda$ as under $\mu$.

\section{Proof}

This follows from the asymptotic independence assertions of Theorem 5.3. Consider first the random variable $\tilde{U}_{T}$ : to show that it converges in distribution under $\lambda$, we must prove that for any bounded, continuous test function $\psi: \mathbb{R} \rightarrow \mathbb{R}$, the expectations $E_{\lambda} \psi\left(\tilde{U}_{T}\right)$ converge as $T \rightarrow \infty$. But since $d \lambda / d \mu$ is a bounded, continuous function, the convergence (46)-(47) and the asymptotic independence of $x$ and $\tilde{U}_{T}(x)$ under $\mu$ imply that

$$
\begin{aligned}
\lim _{T \rightarrow \infty} E_{\lambda} \psi\left(\tilde{U}_{T}\right) & =\lim _{T \rightarrow \infty} E_{\mu} \psi\left(\tilde{U}_{T}\right) \frac{d \lambda}{d \mu} \\
& =\lim _{T \gamma \infty} E_{\mu} \psi\left(\tilde{U}_{T}\right) \lim _{T \rightarrow \infty} E_{\mu} \frac{d \lambda}{d \mu} \\
& =\lim _{T \gamma \infty} E_{\mu} \psi\left(\tilde{U}_{T}\right) .
\end{aligned}
$$

(Note: This holds in both the case where $h_{+}$is cohomologous to a scalar multiple of $F$ and the case where it is not.) A similar argument, using Proposition 4.2 and Propo- 
sition 4.3, proves that the random variables $R_{T}$ and $\tilde{\tau}_{T}$ converge in distribution under $\lambda$ to the same limit distributions as under $\mu$, and that the various random variables, vectors, and sequences are asymptotically independent.

This result will suffice to deduce limit results about continuous-time $U$-statistics in suspension flows under suspensions of Gibbs states (see Section 4.3) from corresponding results about discrete-time $U$-statistics in shifts of finite type. For dealing with measures like the uniform distribution on the set of periodic orbits of minimal period at most $T$, the following variant of Corollary 5.5 will be needed.

\section{COROLLARY 5.6}

Let $\mu=\mu$ be a Gibbs state, and let $h: \Sigma \times \Sigma \rightarrow \mathbb{R}$ be a function that satisfies Hypothesis 5.1 relative to $\mu$. Let $\left\{\lambda_{T}\right\}_{T \geq 1}$ be a family of probability measures on $\Sigma$ such that as $T \rightarrow \infty$,

$$
\frac{d \lambda_{T}}{d \mu}(x) \sim g_{1}(x) g_{2}\left(\sigma^{\tau_{T}(x)} x\right) g_{3}\left(R_{T}(x)\right),
$$

where $g_{1}, g_{2}: \Sigma \rightarrow[0, \infty)$ and $g_{3}:[0, \infty)$ are nonnegative, bounded, continuous functions not depending on $T$, such that $g_{3}$ is strictly positive on an interval and $g_{2}, g_{3}$ both have positive expectation under $E_{\mu}$. Then as $T \rightarrow \infty$, the joint distribution of $\tilde{U}_{T}, \tilde{\tau}_{T}, R_{T}, x$, and $\sigma^{\tau(x)} x$ under $\lambda_{T}$ converges. Moreover, the limiting joint distribution of $\tilde{U}_{T}$ and $\tilde{\tau}_{T}$ is the same as under $\mu$.

\section{Proof}

The proof is virtually the same as that of Corollary 5.5. In particular, for any bounded, continuous test functions $\psi_{1}, \psi_{2}, \psi_{3}: \mathbb{R} \rightarrow \mathbb{R}$ and $\psi_{4}, \psi_{5}: \Sigma \rightarrow \mathbb{R}$,

$$
\begin{aligned}
& \lim _{T \rightarrow \infty} E_{\lambda_{T}} \psi_{1}\left(R_{T}\right) \psi_{2}\left(\tilde{U}_{T}\right) \psi_{3}\left(\tilde{\tau}_{T}\right) \psi_{4}\left(\psi_{5} \circ \sigma^{\tau}\right) \\
& \quad=\lim _{T \rightarrow \infty} E_{\mu} \psi_{1}\left(R_{T}\right) \psi_{2}\left(\tilde{U}_{T}\right) \psi_{3}\left(\tilde{\tau}_{T}\right) \psi_{4}\left(\psi_{5} \circ \sigma^{\tau}\right) g_{1}\left(g_{2} \circ \sigma^{\tau}\right) g_{3}\left(R_{T}(x)\right)
\end{aligned}
$$

by Theorem 5.3, since $g_{1}, g_{2}$, and $g_{3}$ are continuous. Moreover, because the random variables $x, \sigma^{\tau(x)}(x)$, and $R_{T}$ are asymptotically independent of $\left(\tilde{U}_{T}, \tilde{\tau}_{T}\right)$ under $\mu$, they will also be asymptotically independent under $\lambda_{T}$, and the limit distribution of $\left(\tilde{U}_{T}, \tilde{\tau}_{T}\right)$ will be the same as under state $\mu$. However, the limit distributions of $R_{T}$ and $\sigma^{\tau(x)} x$ will in general be different, because unlike the bulk variables $\left(\tilde{U}_{T}, \tilde{\tau}_{T}\right)$ these random variables are highly dependent on the last few coordinates of $x$ before $\tau(x)$. In particular, the limit distribution of $\sigma^{\tau(x)}(x)$ will be "tilted" by the likelihood ratio $g_{2}$ :

$$
\lim _{T \rightarrow \infty} E_{\lambda}\left(\psi \circ \sigma^{\tau}\right)=\lim _{T \rightarrow \infty} E_{\mu}\left(\psi \circ \sigma^{\tau}\right)\left(g_{2} \circ \sigma^{\tau}\right)=E_{\mu} \psi g_{2}
$$




\section{Remark 5.7}

The corollary remains true if it is only assumed that $g_{3}$ is piecewise continuous, in particular, if $g_{3}$ is the indicator function of a bounded interval $[a, b]$. This can be proved by a routine sandwiching argument, using the fact that $\mu\left\{x: R_{T}(x) \in(b-\right.$ $\varepsilon, b+\varepsilon)\}=O(\varepsilon)$, by the renewal theorem (Proposition 4.2). Observe that for this, the standing assumption that $F$ is nonarithmetic is essential.

\section{Cohomology and Hoeffding projections in constant and variable negative cur- vature}

Theorem 5.3 shows that, in general, the large-time behavior of the distribution of a $U$-statistic is dependent on the Hoeffding projection $h_{+}(x)$ of the kernel $h(x, y)$. In particular, if $h_{+}$is cohomologous to a scalar multiple of the height function $F$, then the fluctuations of the $U$-statistic are of order $T$, but otherwise are of order $T^{3 / 2}$. Thus, in any application of the theorem it will be necessary to determine whether or not the Hoeffding projection is cohomologous to a scalar multiple of $F$. In this section we will show that for the function $h(x, y)$ in the representation (25) of the self-intersection count for geodesics on a surface of negative curvature, the factor that determines this is whether or not the curvature is constant.

\subsection{Crossing intensities}

First we make a simple observation about the asymptotic frequencies of intersections of a random geodesic with a fixed geodesic segment (see [8], [7] for far-reaching extensions and consequences of this observation). Fix a (compact) geodesic segment $\alpha$ on $\Upsilon$ (e.g., a [prime] closed geodesic), and for any geodesic ray $\gamma(t)=\gamma(t ; x, \theta)$ let $N_{t}(\alpha ; \gamma)$ be the number of transversal intersections of $\alpha$ with the segment $\gamma([0, t])$.

\section{PROPOSITION 6.1}

Assume that $\Upsilon$ is a compact surface with a Riemannian metric of (possibly variable) negative curvature, and let $v$ be any ergodic, invariant probability measure for the geodesic flow on $S \Upsilon$. Then for each geodesic segment $\alpha$ there is a positive constant $\kappa(\alpha ; \nu)$ such that for $v$-almost every initial vector $(x, \theta)$, the geodesic ray $\gamma$ with initial tangent vector $(x, \theta)$ satisfies

$$
\lim _{t \rightarrow \infty} \frac{N_{t}(\alpha ; \gamma)}{t}=\kappa(\alpha ; \nu)
$$

\section{Proof}

This is a straightforward application of Birkhoff's ergodic theorem. Fix $\varepsilon>0$ sufficiently small that any geodesic segment of length $2 \varepsilon$ can intersect $\alpha$ transversally at most once, and denote by $G$ the set of all unit vectors $(x, \theta) \in S \Upsilon$ such that the geodesic segment $\gamma([-\varepsilon, \varepsilon],(x, \theta))$ crosses $\alpha$ (transversally). Define $g=(2 \varepsilon)^{-1} I_{G}$, 
where $I_{G}$ is the indicator function of $G$. Then

$$
\left|\int_{0}^{t} g\left(\gamma_{s}\right) d s-N_{t}(\alpha ; \gamma)\right| \leq 2
$$

the error \pm 2 enters only because the first and last crossing might be incorrectly counted. Thus, the result follows from Birkhoff's theorem.

Lemma 2.2 (see also [7]) implies that if $v=v_{L}$ is the normalized Liouville measure, then for every geodesic segment $\alpha$,

$$
\kappa\left(\alpha ; v_{L}\right)=\kappa \Upsilon|\alpha|,
$$

where $|\alpha|$ is the length of $\alpha$ and $\kappa_{\Upsilon}$ is as in relation (1). On the other hand, [29, Théorème 2] implies that if two ergodic, invariant probability measures $v, v^{\prime}$ have the same intersection statistics, that is, if

$$
\kappa(\alpha ; v)=\kappa\left(\alpha ; v^{\prime}\right) \quad \text { for all closed geodesics } \alpha,
$$

then $v=v^{\prime}$. Now let $v_{\max }$ be the maximum entropy-invariant measure for the geodesic flow. If $\Upsilon$ has constant negative curvature, then $v_{\max }=v_{L}$, but if $\Upsilon$ has variable negative curvature, then $v_{\max } \neq v_{L}$ (and in fact $v_{\max }$ and $v_{L}$ are mutually singular). This proves the following corollary.

\section{COROLLARY 6.2}

If $v$ is an ergodic, invariant probability measure for the geodesic flow such that the ratio $\kappa(\alpha ; v) /|\alpha|$ has the same value for all closed geodesics $\alpha$, then $v=v_{L}$. Consequently, in order for the ratio $\kappa\left(\alpha ; v_{\max }\right) /|\alpha|$ to have the same value for all closed geodesics $\alpha$, it is necessary and sufficient that the surface $\Upsilon$ have constant negative curvature.

\subsection{Hoeffding projection of the self-intersection kernel}

According to the results of Section 3, the geodesic flow on $S \Upsilon$ is semiconjugate to the suspension flow $\left(\Sigma_{F}, \phi_{t}\right)$ over a shift $(\Sigma, \sigma)$ with Hölder-continuous height function $F$. By Proposition 3.7, the self-intersection counts for closed geodesics and geodesic segments are given by equations (25) and (28), with $h=h^{r}$ for any $0 \leq r<$ $\min F$.

Fix a Gibbs state $\mu$ on $\Sigma$, and let $\lambda$ be the projection to $\Sigma$ of the suspension measure $\mu^{*}$, that is, the absolutely continuous probability measure defined by

$$
\lambda(A)=E_{\mu}\left(I_{A} F\right) / E_{\mu} F .
$$


PROPOSITION 6.3

The Hoeffding projection $h_{+}$of the function $h$ relative to the measure $\lambda$ is cohomologous to a scalar multiple of the height function $F$ if and only if the suspension measure $\mu^{*}$ is the pullback of the Liouville measure on $S \Upsilon$.

\section{Proof}

For any $x \in \Sigma$, the value $h_{+}(x)$ is the probability that the geodesic segments $p \circ$ $\pi\left(\mathcal{F}_{x}\right)$ and $p \circ \pi\left(\mathcal{F}_{y}\right)$ of the suspension flow will intersect when $y$ is randomly chosen according to the law $\lambda$. (By Lemma 3.5, we can assume that the symbolic dynamics have been refined so that any two such segments can intersect at most once.) In order that $h_{+}$be cohomologous to $c F$, it is necessary and sufficient (see [11, Theorem 1.28]) that these two functions sum to the same value over every periodic orbit of the shift; that is, if for every periodic sequence $x \in \Sigma$ with period (say) $n=n(x)$,

$$
\sum_{j=0}^{n-1} h_{+}\left(\sigma^{j} x\right)=\sum_{j=0}^{n-1} c F\left(\sigma^{j} x\right) .
$$

The left-hand side is the expected number of intersections of the closed geodesic $p \circ \pi\left(\mathscr{F}_{x}\right)$ with a geodesic segment $p \circ \pi\left(\mathscr{F}_{y}\right)$ gotten by projecting a fiber $\mathscr{F}_{y}$ of the suspension flow chosen randomly according to the law $y \sim \lambda$. By the ergodic theorem, this expectation is the long-run frequency (per time $E_{\mu} F$ ) of intersections of a randomly chosen geodesic with the closed geodesic $\alpha=p \circ \pi\left(\mathcal{F}_{x}\right)$, and therefore coincides with (a scalar multiple of) $\kappa\left(\alpha ; p_{*} \lambda\right)$. On the other hand, the right-hand side of (59) is just $c$ times the length of $\alpha$. By Corollary 6.2, the two sides of (59) coincide for all $\alpha$ if and only if the projection $p_{*} \lambda$ is the Liouville measure.

\section{Verification of Hypothesis 5.1}

To deduce Theorem 1.1 from the results of Section 5, it will be necessary to show that the relevant function $h$ in the representation (25) satisfies Hypothesis 5.1. For the functions $h$ and $h^{r}$ defined in Section 3, the properties (H0)-(H2) hold trivially, so only statement (H3) of Hypothesis 5.1 warrants consideration. The purpose of this section is to prove that for any Gibbs state $\mu$ there exist values of $r$ such that the function $h^{r}$ defined in Remark 3.8 meets the requirement (H3).

By Proposition 3.2 and Corollary 3.4, for any compact, negatively curved surface $\Upsilon$ the geodesic flow on $S \Upsilon$ is semiconjugate to a suspension flow $\left(\Sigma_{F}, \phi_{t}\right)$ over a topologically mixing shift of finite type $(\Sigma, \sigma)$ with a Hölder-continuous height function $F$. This semiconjugacy is one-to-one except on a set of first category, and both the Liouville measure and the maximum entropy measure for the geodesic flow pull back to suspensions of Gibbs states on $\Sigma$. Furthermore, points $x \in \Sigma$ of the underlying shift are mapped to pairs of points $\xi_{+}\left(x^{+}\right), \xi_{-}\left(x^{-}\right)$on $\partial \mathbb{D}$ in such a way that 
the suspension flow orbit through $(x, 0)$ is mapped to the geodesic whose $L$-lift to the Poincaré plane has endpoints $\xi_{+}\left(x^{+}\right), \xi_{-}\left(x^{-}\right)$; and this mapping sends cylinder sets to $\operatorname{arcs}$ of $\delta \mathbb{D}$ satisfying (D) and (E) of Proposition 3.2. By Lemma 3.5, for any small $\varepsilon>0$ the symbolic dynamics admits a refinement for which the height function $F$ satisfies $F<\varepsilon$. By Proposition 3.7, the self-intersection counts for closed geodesics and geodesic segments are given by equations (25) and (28), with $h=h^{r}$ for any $0 \leq r<\min F$. The function $h^{r}$ decomposes as in equation (31).

\section{PROPOSITION 7.1}

For any Gibbs state $\mu$, the functions $h_{m}^{r}$ satisfy (H3) of Hypothesis 5.1 relative to $\mu$ for almost every $r$ in some interval $\left[0, r_{*}\right]$ of positive length $r_{*}$.

The remainder of this section is devoted to the proof of this proposition. The key is the property (A3) from Remark 3.8, which asserts that there exists $\varrho<1$ such that $h_{n}^{r} \neq 0$ for some $n \geq m$ only if the geodesic segments corresponding to the suspension flow segments $\mathcal{F}_{x}^{r}$ and $\mathcal{F}_{y}^{r}$ (see equation (30)) intersect either at an angle less than $C^{\prime} \varrho^{m}$ or at a point within distance $C^{\prime} \varrho^{m}$ of one of the endpoints of the two geodesic segments.

For any integers $m, k \geq 1$, define

$$
\begin{aligned}
A_{m, k}^{r}= & \left\{x \in \Sigma: p \circ \pi\left(\mathcal{F}_{x}^{r}\right) \text { and } p \circ \pi\left(\mathcal{F}_{\sigma^{k}}^{r}\right) \text { intersect at angle }<\varrho^{m}\right\} \text { and } \\
B_{m, k}^{r}= & \left\{x \in \Sigma: p \circ \pi\left(\mathcal{F}_{x}^{r}\right) \text { and } p \circ \pi\left(\mathcal{F}_{\sigma^{k}}^{r}\right) \text { intersect at distance }<\varrho^{m}\right. \\
& \text { of } p(\pi(x, 0)) \text { or } p(\pi(x, F(x)-r))\} .
\end{aligned}
$$

To show that (H3) of Hypothesis 5.1 holds relative to a Gibbs state $\mu$, it is enough to show that there exist $C<\infty$ and $\beta<1$ such that, for all sufficiently large $m$ and all $k \neq 0$,

$$
\mu\left(A_{m, k}^{r}\right)+\mu\left(B_{m, k}^{r}\right) \leq C \beta^{m} .
$$

We will show in Lemmas 7.4 and 7.7 that each of the probabilities $\mu\left(A_{m, k}^{r}\right)$ and $\mu\left(B_{m, k}^{r}\right)$ is exponentially decaying in $m$, uniformly in $k$, for almost every $r$ in a small interval $\left[0, r_{*}\right]$ of positive length.

\subsection{Intersections in small balls}

We begin with $\mu\left(B_{m, k}^{r}\right)$. The strategy for bounding this will be to first handle the case $|k| \leq \exp \{\varepsilon m\}$ for small $\varepsilon>0$ by a density argument, and then the case $|k|>$ $\exp \{\varepsilon m\}$ by using the exponential mixing property (34) of Gibbs states. 
LEMMA 7.2

If $0<\varrho<\alpha<1$, then for any Gibbs state $\mu$ and almost every $r<\min F / 3$, if $m$ is sufficiently large, then

$$
\mu\left(B_{m, k}^{r}\right) \leq \alpha^{m} \quad \text { for all }|k| \leq(\alpha / \varrho)^{m / 2}, k \neq 0 .
$$

\section{Proof}

Without loss of generality we can assume (see Lemma 3.5) that no two geodesic segments of length less than $2 \max F$ intersect transversally more than once. For $x \in$ $\Sigma$, let $B_{m, k}(x)$ be the set of $r \in[0, F(x)+F(\sigma x)]$ such that the geodesic segments $p \circ \pi\left(\mathcal{F}_{x} \cup \mathcal{F}_{\sigma x}\right)$ and $p \circ \pi\left(\mathcal{F}_{\sigma^{k}} \cup \mathcal{F}_{\sigma^{k+1}}\right)$ intersect at distance less than $\varrho^{m}$ of $p \circ$ $\pi(x, r)$. Because there is at most one intersection, the Lebesgue measure of $B_{m, k}(x)$ is less than $2 \varrho^{m}$. Since $x \in B_{m, k}^{r}$ implies that $r \in B_{m, k}(x)$, it follows by Fubini's theorem that for any $\alpha \in(\varrho, 1)$,

$$
\begin{aligned}
m_{\text {Leb }}\{r & \left.\in[0, \min F / 3]: \mu\left(B_{m, k}^{r}\right) \geq \alpha^{m}\right\} \leq 2(\varrho / \alpha)^{m} \\
\Longrightarrow & m_{\text {Leb }}\left\{r \in[0, \min F / 3]: \mu\left(B_{m, k}^{r}\right) \geq \alpha^{m} \text { for some }|k| \leq(\alpha / \varrho)^{1 / 2}\right\} \\
& \leq 2(\varrho / \alpha)^{m / 2} .
\end{aligned}
$$

Since $\sum_{m}(\varrho / \alpha)^{m / 2}<\infty$, it follows by the Borel-Cantelli lemma that for almost every $r \in[0, \min F / 3]$, the inequality (61) holds for all sufficiently large $m$.

\section{LEMMA 7.3}

For any Gibbs state $\mu$ on $\Sigma$ and any $T<\infty$, there exist $\delta=\delta(\mu, T)>0$ and $C=C_{T, \mu}>0$ with the following property: for any ball $B$ in $\Upsilon$ of sufficiently small diameter $\varepsilon>0$,

$$
\mu\left\{x \in \Sigma: p \circ \pi\left(\left\{\phi_{t}(x, 0)\right\}_{0 \leq t \leq T}\right) \text { intersects } B\right\} \leq C \varepsilon^{\delta} .
$$

\section{Proof}

In the special case where the suspension $\mu_{*}$ of the Gibbs state $\mu$ (see equation (37)) is the pullback of the Liouville measure, this is apparent from purely geometric considerations, as we now show. We may assume that for $\varepsilon$ sufficiently small, the image $p \circ \pi\left(\mathcal{F}_{x}\right)$ of a fiber can intersect a ball of radius $2 \varepsilon$ at most once. Since the surface area measure of a ball $B(x, \varepsilon)$ of radius $\varepsilon$ is comparable to $\varepsilon^{2}$, the ergodic theorem implies that the long-run fraction of time spent in $B(x, 2 \varepsilon)$ is almost surely $K \varepsilon^{2}$, for a constant $K$ independent of $\varepsilon$. On each visit to $B(x, \varepsilon)$, a geodesic must spend time at least $K^{\prime} \varepsilon$ in $B(x, 2 \varepsilon)$. Consequently, the long-run fraction of the sequence of fibers $p \circ \pi\left(\widetilde{F}_{\sigma^{n} x}\right)$ on a geodesic that visits $B(x, \varepsilon)$ is less than $K \varepsilon / K^{\prime}$; thus, by the ergodic theorem, (62) holds with $\delta=1$. 
Unfortunately, for arbitrary Gibbs states there is no simple relation between the surface area and the Gibbs measure, so a different argument is needed. Consider first the case of a Riemannian metric with constant curvature -1 . Recall that in this case, the surface $\Upsilon$ can be identified with a compact polygon $\mathcal{P}$ in the Poincare disk $\mathbb{D}$ whose edges are pasted together in pairs. With this identification, any ball $B$ in $\Upsilon$ corresponds to a ball of the same radius in the interior of $\mathcal{P}$, provided that this ball does not intersect $\partial \mathcal{P}$, or otherwise a union of at most $4 g$ sectors of balls of the same radius, where $4 g$ is the number of sides of $\mathcal{P}$. Thus, a geodesic segment of length less than $\min F$ that intersects $B$ will lift to a geodesic segment in $\mathbb{D}$ that intersects one of up to $4 g$ balls of the same radius, all with centers in the closure of $\mathcal{P}$. Consequently, a geodesic segment of length $T$ in $\Upsilon$ that intersects a ball of radius $\varepsilon$ in $\Upsilon$ lifts to a geodesic segment in $\mathbb{D}$ that intersects one of up to $4 g T$ balls of the same radius, all with centers at distance no more than $T$ from $\mathcal{P}$.

Fix a point $\zeta_{-} \in \partial \mathbb{D}$ on the circle at infinity, and consider the set of all geodesics in $\mathbb{D}$ with $\zeta_{-}$as an endpoint (as $t \rightarrow-\infty$ ) that intersect a ball $B$ of radius $\varepsilon$ with center in $\mathcal{P} \cup \partial \mathcal{P}$. For any such geodesic, the second endpoint $\zeta_{+}$on $\partial \mathbb{D}$ is constrained to lie in an arc $J\left(\zeta_{-}, B\right)$ of length at most $K \varepsilon$, where $K$ is a constant that does not depend on $\zeta_{-}$or on the center of $B$. Recall (see Proposition 3.2) that specification of the endpoint $\zeta_{-}$of a geodesic is equivalent (except on a set of first category) to specification of the backward coordinates $x^{-}$of the sequence $x \in \Sigma$ that represents the geodesic; and similarly, specification of the endpoint $\zeta_{+}$is equivalent to specification of the forward coordinates $x^{+}$. By (D) and (E) of Proposition 3.2, it follows that constraining $\zeta_{+}$to lie in an arc of length at most $K \varepsilon$ has the effect of constraining its forward itinerary $x^{+}$to lie in a union of one or two cylinder sets $\Sigma_{[0, m]}^{+}(y)$ with $m=K^{\prime} \log \varepsilon^{-1}$. Now for any Gibbs state $\mu$ there exists $\beta<1$ such that the $\mu$-measure of any cylinder set $\Sigma_{[1, m]}(x)$ is less than $\beta^{m}$. Moreover, by inequality (33), the conditional measure $\mu\left(\cdot \mid x^{-}\right)$given the past is dominated by a constant multiple of the unconditional measure $\mu$. Thus, if $G^{\varepsilon}(B)$ denotes the set of all $x \in \Sigma$ such that the suspension flow segment $\left\{\phi_{t}(x, 0)\right\}_{0 \leq t \leq T}$ lifts to a geodesic segment that intersects $B$, then

$$
\mu\left(G^{\varepsilon}(B)\right)=E_{\mu}\left(E_{\mu}\left(I_{G^{\varepsilon}(B)} \mid \mathcal{B}_{(-\infty, 0]}\right)\right) \leq \beta^{m} .
$$

This implies (62) in the constant curvature case.

This argument extends to metrics of variable negative curvature, with the aid of the structural stability results of Section 3.2. Let $\varrho_{1}$ be a metric of variable negative curvature, and let $\varrho_{0}$ be a metric of curvature -1 . Recall that the $\varrho_{1}$-geodesic flow is orbit-equivalent, by a Hölder-continuous mapping $\Phi: S \Upsilon \rightarrow S \Upsilon$, to the $\varrho_{0}$-geodesic flow, and that the homeomorphism $\Phi$ lifts to a homeomorphism $\tilde{\Phi}: S \mathbb{D} \rightarrow S \mathbb{D}$ of the universal cover. Each $\varrho_{0}$-geodesic in $\mathbb{D}$ corresponds under $\tilde{\Phi}$ to a $\varrho_{1}$-geodesic, and these have the same endpoints on $\partial \mathbb{D}$ and the same symbolic representation $x \in \Sigma$. Because $\tilde{\Phi}$ is Hölder, constraining a $\varrho_{1}$-geodesic to pass through a $\varrho_{1}$-ball of radius $\varepsilon$ 
forces the corresponding $\varrho_{0}$-geodesic to pass through a $\varrho_{0}$-ball of radius $\varepsilon^{\alpha}$, for some $\alpha>0$ depending on the Hölder exponent and all $\varepsilon$ sufficiently small. Therefore, the problem reduces to the constant curvature case.

\section{LEMMA 7.4}

If $\varrho<1$, then for any Gibbs state $\mu$ and for almost every $0 \leq r<\min F / 3$, there exists $\alpha<1$ such that if $m$ is sufficiently large, then

$$
\mu\left(B_{m, k}^{r}\right) \leq \alpha^{m} \text { for all } k \neq 0
$$

\section{Proof}

Lemma 7.2 implies that if $\varrho \leq \alpha<1$, then for almost every $r<\min F / 3$ the inequality (63) holds for all $|k|<(\alpha / \varrho)^{m / 2}$. We will show that for every $0 \leq r<\min F / 3$ the inequality (63) also holds for $|k| \geq(\alpha / \varrho)^{m / 2}$; for this we will appeal to the exponential mixing inequality (33), using Lemma 7.3 to control the first moment. The proof will rely on the following elementary geometric fact. For any compact Riemannian manifold $\mathcal{M}$ there exists $\kappa<\infty$ (depending on the metric) such that for every sufficiently small $\varepsilon>0$ there is a finite set of points $z_{1}, z_{2}, \ldots, z_{n}$ such that every $x \in \mathcal{M}$ is within distance $\varepsilon$ of some $z_{i}$ but is within distance $6 \varepsilon$ of at most $\kappa$ distinct points $z_{i}$. Call such a collection of points $z_{i}$ an efficient $\varepsilon$-net.

In order that $x \in B_{m, k}^{r}$, it is necessary that the geodesic segment $p \circ \pi\left(\mathcal{F}_{\sigma^{k} x}^{r}\right)$ intersects either the ball of radius $\varrho^{m}$ centered at $p \circ \pi\left(\phi_{r}(x)\right)$, or the ball of radius $\varrho^{m}$ centered at $p\left(\phi_{F(x)-r}(x)\right)$, or both. Let $z_{1}, \ldots, z_{n}$ be an efficient $\varrho^{m}$-net, and let $B\left(z_{i}, 3 \varrho^{m}\right)$ be the ball of radius $3 \varrho^{m}$ centered at $z_{i}$. Then

$$
B_{m, k}^{r} \subset \bigcup_{i=1}^{n} H_{i, m}^{r} \cap G_{i, m, k}^{r}
$$

where $H_{i, m}^{r}$ is the set of all $x \in \Sigma$ such that $p \circ \pi(x, r) \in B\left(z_{i}, 3 \varrho^{m}\right)$ and where $G_{i, m, k}$ is the set of all $x \in \Sigma$ such that the geodesic segment $p \circ \pi\left(\widetilde{F}_{\sigma^{k} x} \cup \mathcal{F}_{\sigma^{k+1}}\right)$ intersects $B\left(z_{i}, 3 \varrho^{m}\right)$. (This is because $\mathcal{F}_{y}^{r} \subset \mathcal{F}_{y} \cup \mathcal{F}_{\sigma y}$.) Since $z_{1}, \ldots, z_{n}$ is an efficient $\varrho^{m}$-net, at most $\kappa$ of the events $H_{i, m}^{r}$ can occur together; consequently,

$$
\begin{aligned}
\mu\left(B_{m, k}^{r}\right) & \leq \sum_{i=1}^{n} \mu\left(H_{i, m}^{r} \cap G_{i, m, k}\right) \\
& =\sum_{i=1}^{n} \mu\left(H_{i, m}^{r}\right) \mu\left(G_{i, m, k} \mid H_{i, m}^{r}\right) \\
& \leq \kappa \max _{i \leq n} \mu\left(G_{i, m, k} \mid H_{i, m}^{r}\right) .
\end{aligned}
$$


Thus, it remains to bound the conditional probabilities $\mu\left(G_{i, m, k} \mid H_{i, m}^{r}\right)$ for $|k|>$ $(\alpha / \varrho)^{m / 2}$.

For each $i$, let $0 \leq \psi_{i} \leq 1$ be a smooth function on $\Upsilon$ with Lipschitz norm less than $6 \varrho^{-m}$ that takes the value 1 on $B\left(z_{i}, 3 \varrho^{m}\right)$ and 0 on the complement of $B\left(z_{i}, 6 \varrho^{m}\right)$. For each $x \in \Sigma$ and $0 \leq r \leq \min F / 3$, define

$$
\begin{aligned}
g_{i, m}(x) & =\max _{0 \leq s \leq F(x)+F(\sigma x)} \psi_{i}(p \circ \pi(x, s)) \text { and } \\
h_{i, m}^{r}(x) & =\psi_{i}(p \circ \pi(x, r)) .
\end{aligned}
$$

Since the projection $p \circ \pi$ is $\delta$-Hölder-continuous for some exponent $\delta$, both $g_{i, m}$ and $h_{i, m}^{r}$ have $\delta$-Hölder norms bounded by $6\|p \circ \pi\|_{\delta} \varrho^{-m}$. Therefore, the exponential mixing inequality (34) implies that for some $C<\infty$ and $0<\beta<1$ independent of $i$, $m, k$, and $r$,

$$
\begin{aligned}
\mu\left(G_{i, m, k} \cap H_{i, m}^{r}\right) & \leq E_{\mu}\left(g_{i, m} \circ \sigma^{k}\right) h_{i, m}^{r} \\
& \leq E_{\mu} g_{i, m} E_{\mu} h_{i, m}^{r}+C \beta^{k} \varrho^{-m}
\end{aligned}
$$

For $|k|>(\alpha / \varrho)^{m / 2}$, the second term is super-exponentially decaying in $m$. But Lemma 7.3 implies that the expectation $E_{\mu} g_{i, m}$ is bounded by $\left(6 \varrho^{m}\right)^{q}$ for some $q>0$, and so the result now follows.

\subsection{Intersections at small angles}

Next we must show that the events $A_{m, k}^{r}$ have uniformly exponentially decaying probabilities, in the sense of (60). The strategy here will be to show that if two geodesic segments corresponding to distinct fibers of the suspension flow cross at a small angle, then it will be impossible for their successors to cross for a long time. This fact, coupled with the ergodic theorem, will imply that the probability of a crossing at a small angle must be small. The key geometric fact is as follows (see also [6]).

\section{LEMMA 7.5}

For any $\kappa>0$ sufficiently small and any $\varrho<1$, there exists $C<\infty$ such that for all large $m \geq 1$ the following holds. If two geodesic segments $\gamma([0,2 \kappa], x)$ and $\gamma([0,2 \kappa]$, $y$ ) of length $2 \kappa$ cross transversally at an angle less than $\varrho^{m}$, then for every $1 \leq j \leq$ $C m$, the geodesic segments $\gamma([j \kappa, j \kappa+2 \kappa], x)$ and $\gamma([j \kappa, j \kappa+2 \kappa], y)$ do not cross.

\section{Proof}

Let $\kappa>0$ be sufficiently small that no two geodesic segments of length $3 \kappa$ on $\Upsilon$ can cross transversally more than once. Consider lifts $\tilde{\gamma}(t, x)$ and $\tilde{\gamma}(t, y)$ of the geodesic rays $\gamma(t, x)$ and $\gamma(t, y)$ to the universal covering surface $\tilde{\Upsilon}$ whose initial segments $\tilde{\gamma}([0,2 \kappa], \tilde{x})$ and $\tilde{\gamma}([0,2 \kappa], \tilde{y})$ cross transversally at angle less than $\varrho^{m}$. These 
geodesic rays cannot cross again because for any two points in a Cartan-Hadamard manifold there is only one connecting geodesic. Consequently, if for some $j$ the geodesic segments $\gamma([j \kappa, j \kappa+2 \kappa], x)$ and $\gamma([j \kappa, j \kappa+2 \kappa], y)$ were to cross, then their lifts $\tilde{\gamma}([j \kappa, j \kappa+2 \kappa], x)$ and $\tilde{\gamma}([j \kappa, j \kappa+2 \kappa], y)$ would contain points $\tilde{w}, \tilde{z}$, respectively, such that $\tilde{z}=g \tilde{w}$ for some element $g \neq 1$ of the group of deck transformations. However, if the initial angle of intersection is less than $\varrho^{m}$, then the geodesic rays $\tilde{\gamma}(t, x)$ and $\tilde{\gamma}(t, y)$ cannot diverge by more than $\varepsilon$ for time $C m$, where $C$ is a constant determined by $\varepsilon$ and the curvature of $\Upsilon$ (which is bounded, since $\Upsilon$ is compact). If $\varepsilon>0$ and $\kappa>0$ are sufficiently small, then this would preclude the existence of points $\tilde{w}, \tilde{z}$ such that $\tilde{w}=g \tilde{z}$ for some $g \neq 1$.

Because the semiconjugacy $\pi: \Sigma_{F} \rightarrow S \Upsilon$ is not one-to-one, two orbits of the geodesic flow can remain close for a long time but have symbolic representations that are not close. The next lemma shows that, at least for the symbolic dynamics constructed by Series (see Section 3.2) and refinements such as that described in the proof of Lemma 3.5, this event has small probability under any Gibbs state.

Fix $\alpha>0$ and $\varepsilon>0$, and for each $m \geq 1$ let $D_{m}=D_{m}^{\alpha, \varepsilon}$ be the set of all sequences $x \in \Sigma$ such that there exists $(y, s) \in \Sigma_{F}$ satisfying

$$
\begin{aligned}
\operatorname{distance}\left(\pi\left(\phi_{t}(x, 0)\right), \pi\left(\phi_{t}(y, s)\right)\right) & \leq \varepsilon \quad \text { for all }|t| \leq e^{\alpha m} \quad \text { and } \\
x_{i} & \neq y_{i} \quad \text { for some }|i| \leq m .
\end{aligned}
$$

\section{LEMMA 7.6}

Let $\mu$ be any Gibbs state. Then for all sufficiently small $\varepsilon>0$ and all sufficiently large $\alpha$ there exist $\beta<1$ and $C<\infty$ such that

$$
\mu\left(D_{m}\right) \leq C \beta^{m} \text { for all } m \geq 1 .
$$

\section{Proof}

Recall (see Proposition 3.3 and following) that the geodesic flow with respect to a Riemannian metric of variable negative curvature is orbit-equivalent to the geodesic flow on the same surface but with a Riemannian metric of constant curvature -1 , and that the orbit equivalence is given by a Hölder-continuous mapping $S \Upsilon \rightarrow S \Upsilon$. Therefore, it suffices to prove the lemma for the geodesic flow on a surface of constant curvature -1 . (The conformal deformation of metric might change the values of $\beta$ and $\varepsilon$, but this is irrelevant.)

Suppose, then, that the Riemannian metric has curvature -1 , and that $\pi \circ \phi_{t}(x, 0)$ and $\pi \circ \phi_{t}(y, s)$ are two geodesics on the unit tangent bundle that stay within distance $\varepsilon$ for all $|t| \leq e^{\alpha m}$, for some small $\varepsilon$ and large $\alpha$. Because distinct orbits of the geodesic flow separate exponentially fast (at exponential rate 1 , since the curvature 
is -1$)$, the initial vectors $\pi(x, 0)$ and $\pi(y, s)$ must be within distance $\kappa e^{-\alpha m}$, for some constant $\kappa=\kappa(\varepsilon)>0$ independent of $\alpha$ and $m$ (provided that $m$ is sufficiently large).

Recall that geodesics can be lifted to $S \mathbb{D}$ via the mapping $L$ described in Section 3.2. This mapping has discontinuities only at vectors tangent to one of the sides of the fundamental polygon $\mathcal{P}$, but everywhere else is smooth; consequently, either

(a) $L \circ \pi(x, 0)$ and $L \circ \pi(y, s)$ are within distance $C \kappa e^{-\alpha m}$, or

(b) $L \circ \pi(x, 0)$ is within distance $C \kappa e^{-\alpha m}$ of a vector tangent to one of the sides of $\mathcal{P}$.

In case (a), the lifted geodesics must have endpoints on $\partial \mathbb{D}$ that are within distance $C^{\prime} \kappa e^{-\alpha m}$; in case (b), the lifted geodesics must have endpoints within distance $C^{\prime} \kappa e^{-\alpha m}$ of the endpoints on $\partial \mathbb{D}$ of one of the geodesics that bound $\mathcal{P}$ (recall that the sides of $\mathcal{P}$ are geodesic arcs). In either case, if $x$ and $y$ disagree in some coordinate $|i| \leq m$, then by Proposition 3.2 at least one of the endpoints of the geodesic $L \circ \pi \circ \phi_{t}(x, 0)$ must be within distance $C^{\prime} \kappa e^{-\alpha m}$ of one of the endpoints of an arc $J_{k}\left(z^{+}\right)$of some generation $k \leq m$. (Recall that the arcs $J_{k}\left(z^{+}\right)$correspond to cylinder sets $\Sigma_{[0, m]}^{+}\left(z^{+}\right)$.) There are at most $e^{2 A m}$ such endpoints, where $A$ is the number of sides of $\mathcal{P}$.

If $\zeta$ is one of the endpoints of an $\operatorname{arc} J_{k}\left(z^{+}\right)$of generation $k$, then $\zeta$ has two symbolic expansions (i.e., there are two sequences $z^{+}, z_{*}^{+}$that are mapped to $\zeta$ by $\xi$ ). Since the $\operatorname{arcs} J_{k}(\cdot)$ do not shrink faster than exponentially (see item (D) of Proposition 3.2), the forward endpoint $\xi\left(x^{+}\right)$of the geodesic $L \circ \pi \circ \phi_{t}(x, 0)$ will lie within distance $C^{\prime} \kappa e^{-\alpha m}$ of $\xi\left(z^{+}\right)$only if either

$$
x_{i}=z_{i} \quad \forall 0 \leq i \leq C^{\prime \prime} \alpha m \quad \text { or } \quad x_{i}=\left(z_{*}\right)_{i} \quad \forall 0 \leq i \leq C^{\prime \prime} \alpha m,
$$

for a suitable constant $C^{\prime \prime}>0$ not depending on $m$ or $\alpha$. Hence, since a Gibbs state $\mu$ will attach mass at most $e^{-b m}$ to cylinder sets of generation $m$, for some $b=b(\mu)>$ 0 , it follows that

$$
\mu\left(D_{m}\right) \leq C^{\prime \prime \prime} \exp \{-b \alpha m\} \exp \{2 A m\}
$$

By choosing $\alpha>0$ such that $b \alpha>2 A$, we can arrange that (64) holds.

LEMMA 7.7

For any Gibbs state $\mu$ there exists $\beta=\beta(\varrho)<1$ such that, for all sufficiently large $m$ and all $k \neq 0$,

$$
\mu\left(A_{m, k}^{r}\right) \leq \beta^{m}
$$

Proof

By Lemma 7.5, it suffices to show that there exist $\alpha>0, \varepsilon>0$, and $\beta<1$ such that 
for all large $m$,

$$
\mu\left(A_{m, k}^{r} \backslash D_{m}^{\alpha, \varepsilon}\right) \leq \beta^{m} .
$$

Suppose that $x \in A_{m, k}^{r} \backslash D_{m}^{\alpha, \varepsilon}$. Then the geodesic segments $p \circ \pi\left(\mathcal{F}_{x}^{r}\right)$ and $p \circ$ $\pi(\mathcal{F})_{\sigma^{k} x}^{r}$ cross at angle less than $\varrho^{m}$; in particular, there exist $r \leq s_{1} \leq F(x)+F(\sigma x)$ and $r \leq s_{2} \leq F\left(\sigma^{k} x\right)+F\left(\sigma^{k+1} x\right)$ such that $p \circ p\left(x, s_{1}\right)=p \circ \pi\left(\sigma^{k}, s_{2}\right)$. Consequently, for some $\alpha>0$ depending on the curvature of the underlying Riemannian metric,

$$
\operatorname{distance}\left(\pi\left(\phi_{t}\left(x, s_{1}\right)\right), \pi\left(\phi_{t}\left(\sigma^{k}, s_{2}\right)\right)\right) \leq \varepsilon \quad \text { for all }|t| \leq e^{\alpha m} .
$$

Since $x \notin D_{m}^{\alpha, \varepsilon}$, it follows that $x_{i}=x_{i+k}$ for all $|i| \leq m$. The lemma now follows from Lemma 4.1.

\section{Proof of Theorem 1.1}

In this section, we deduce Theorem 1.1 from the results of Section 5 using the symbolic dynamics for the geodesic flow outlined in Section 3. For this symbolic dynamics, the normalized Liouville measure $v_{L}$ pulls back to a measure $\mu^{*}$ on the suspension space $\Sigma$ that is the suspension (see equation (37)) of a Gibbs state $\mu=\mu_{L}$ on $\Sigma$. Proposition 7.1 implies that for any Gibbs state $\mu$ there exist values of $r$ such that the functions $h^{r}$ and $h_{m}^{r}$ in equation (31) satisfy Hypothesis 5.1 for $\lambda=\mu_{L}$, and therefore also satisfy any probability measure $\lambda$ on $\Sigma$ that is absolutely continuous with respect to $\mu_{L}$. Recall from Remark 3.8 that replacing the functions $h, h_{m}$ by $h^{r}, h_{m}^{r}$ is equivalent to moving the Poincaré section of the suspension flow. For notational ease, we will assume henceforth that the cross section has been adjusted in such a way that (H3) holds for $r=0$, and drop the superscript from the functions $h, h_{m}$.

Let $\lambda$ be the projection to $\Sigma$ of the suspension measure $\mu^{*}$, that is, the absolutely continuous probability measure defined by

$$
\lambda(A)=E_{\mu_{L}}\left(I_{A} F\right) / E_{\mu_{L}} F .
$$

By Proposition 6.3, the Hoeffding projection $h_{+}$of the function $h$ relative to the measure $\lambda$ is a scalar multiple of $F$; in particular,

$$
h_{+}=\kappa F,
$$

where $\kappa=1 /(4 \pi|\Upsilon|)$. Consequently, case (46) of Theorem 5.3 applies. Theorem 1.1 would follow immediately from Theorem 5.3 if not for the presence of the "boundary terms" sum $\sum_{1}^{\tau}\left(g_{0}+g_{1}\right)$ in (28), since this is of order $O(T)$. The following lemma will show that this sum, normalized by $T$, converges in distribution as $T \rightarrow \infty$, and that the limits depend only on the initial and final points of the flow segment. 
LEMMA 8.1

For $\lambda$-almost every $x \in \Sigma$, every $0 \leq s \leq F(x)$, and every $0 \leq r \leq F\left(\sigma^{\tau_{T}} x\right)$,

$$
\begin{aligned}
\lim _{T \rightarrow \infty} T^{-1} \sum_{i=1}^{\tau_{T}} g_{0}\left(s, x, \sigma^{i} x\right) & =s \kappa \quad \text { and } \\
\lim _{T \rightarrow \infty} T^{-1} \sum_{i=0}^{\tau_{T}} g_{1}\left(S_{\tau_{T}+1} F(x)-r, x, \sigma^{i} x\right) & =r \kappa
\end{aligned}
$$

where $\kappa=\kappa \Upsilon=1 /(4 \pi|\Upsilon|)$.

\section{Proof}

The relation (67) follows from the results of Section 2 and the ergodic theorem. The sum $\sum_{i=1}^{\tau_{T}} g_{0}\left(s, x, \sigma^{i} x\right)$ counts the number of intersections of the geodesic segment $p \circ \pi\left(\left\{\phi_{t}(x, s)\right\}\right)_{-s \leq t \leq 0}$ with the union of the segments $p \circ p\left(\widetilde{F}_{\sigma^{j}}\right)$ for $0 \leq j \leq$ $\tau_{T}(x)$; this sum can be re-expressed in terms of the intersection kernel $H_{\delta}$ (see Section 2), yielding

$$
\sum_{i=1}^{\tau_{T}} g_{0}\left(s, x, \sigma^{i} x\right)=\lim _{\delta \rightarrow 0} \sum_{i=1}^{[s / \delta]} \sum_{j=1}^{[T / \delta]} H_{\delta}(\tilde{\gamma}(-s+i \delta), \tilde{\gamma}(j \delta))+O(1) .
$$

(The error term accounts for the possibility of an intersection with the geodesic segment corresponding to the final partial fiber, and therefore is either 0 or 1.) For each fixed point $\tilde{\gamma}(-s+i \delta)$, the ergodic theorem and Lemma 2.2 imply that

$$
\lim _{T \rightarrow \infty} T^{-1} \sum_{j=1}^{[T / \delta]} H_{\delta}(\tilde{\gamma}(-s+i \delta), \tilde{\gamma}(j \delta))=\delta^{2} \kappa
$$

almost surely. Letting $\delta \rightarrow 0$, one obtains the first limit in (67). The second limit is obtained in a similar fashion.

\section{Proof of Theorem 1.1}

The measure $\lambda$ is the projection of the suspension measure $\mu_{L}^{*}$, which in turn is the pullback to the suspension space $\Sigma_{F}$ of the Liouville measure on $S \Upsilon$. Thus, if $(x, s) \in \Sigma_{F}$ is randomly chosen with distribution $\mu_{L}^{*}$, then $x$ has distribution $\lambda$ and the normalized vertical coordinate $s / F(x)$ is uniformly distributed on the unit interval $[0,1]$ and is independent of $x$. Hence, Lemma 8.1 implies that if $(x, s)$ has distribution $\mu_{L}^{*}$, then the first normalized boundary sum (67) will converge to $Y F(x) \kappa$, where $Y$ is a uniform $[0,1]$ random variable independent of $x$. Since the double sum $\sum_{0}^{\tau} \sum_{0}^{\tau}$ in the representation (25) depends only on $x$, it follows from Corollary 5.5 that the random variables 


$$
\frac{\sum_{0}^{\tau} \sum_{0}^{\tau} h\left(\sigma^{i} x, \sigma^{j} x\right)-E_{\mu_{L}^{*} h_{+}}}{T} \text { and } \quad \frac{\sum_{i=1}^{\tau_{T}} g_{0}\left(s, x, \sigma^{i} x\right)}{T}
$$

are asymptotically independent as $T \rightarrow \infty$. Similarly, by Corollary 5.5 and the renewal theorem, if $(x, s)$ has distribution $\mu_{L}^{*}$, then the overshoot $R_{T}$ and the terminal state $\sigma^{\tau} x$ are asymptotically independent of $x, Y$ and of the double sum $\sum_{0}^{\tau} \sum_{0}^{\tau}$, and so by Lemma 8.1, the second boundary sum

$$
T^{-1} \sum_{i=0}^{\tau_{T}} g_{1}\left(S_{\tau_{T}+1} F(x)-t+s, x, \sigma^{i} x\right)
$$

is asymptotically independent of the other two sums in (28). Theorem 1.1 now follows, as Lemma 8.1 implies that the normalized boundary-term sum converges almost surely and Corollary 5.5 implies that the normalized sum $\sum_{1}^{\tau} \sum_{1}^{\tau}$ converges in distribution.

\section{Proof of Theorem 1.2}

\subsection{Local self-intersection counts}

Recall that for any smooth function $\varphi: \Upsilon \rightarrow \mathbb{R}_{+}$, the $\varphi$-localized self-intersection counts of a geodesic $\gamma$ are defined by

$$
N_{\varphi}(T)=N_{\varphi}(T ; \gamma)=\sum_{i=1}^{N(T)} \varphi\left(x_{i}\right)
$$

where $N(T)=N(T ; \gamma)$ is the number of self-intersections of the geodesic segment $\gamma[0, T]$, and $x_{i}$ are the locations of the self-intersections on $\Upsilon$. Like the global selfintersection counts, these can be expressed as sums of suitable functions defined on a shift of finite type. Let $\left(\Sigma_{F}, \phi_{t}\right)$ and $(\Sigma, \sigma)$ be the suspension flow and shift of finite type, respectively, provided by Proposition 3.7. Define a function $h_{\varphi}: \Sigma \times \Sigma \rightarrow \mathbb{R}_{+}$ by setting

$$
h_{\varphi}(x, y)=\varphi(z(x, y)) h(x, y)
$$

where $h=1$ if the geodesic segments corresponding to the suspension flow segments $\mathcal{F}_{x}$ and $\mathcal{F}_{y}$ intersect at a point $z=z(x, y) \in \Upsilon$, and $h=0$ if these segments do not intersect. By the same reasoning as in equation (28),

$$
N_{\varphi}(T ; \gamma)=\frac{1}{2} \sum_{i=1}^{\tau_{T}} \sum_{i=1}^{\tau_{T}} h_{\varphi}\left(\sigma^{i} x, \sigma^{j} x\right)+O(T)
$$

The error term accounts for intersections with the geodesic segments corresponding to the first and last partial fibers (see equation (28)), of which there are at most $O(T)$. 
Because the normalization in Theorem 1.2 (see relation (4)) entails division by $T^{3 / 2}$, the error term in (70) can be ignored.

The proof of Theorem 1.2, like that of Theorem 1.1 in Section 8, will rely on Corollary 5.5. Once again, let $\mu^{*}$ be the pullback of the Liouville measure to $\Sigma_{F}$; recall that this is the suspension of a Gibbs state $\mu$ for the shift. Let $\lambda$ be the projection of $\mu^{*}$ to $\Sigma$, as defined by (58). This is absolutely continuous with respect to $\mu$, so by Corollary 5.5 the conclusions of Theorem 5.3 remain valid for $\lambda$. We must show first that the function $h_{\varphi}$ satisfies Hypothesis 5.1 with respect to $\lambda$, and second that it is the second case of Theorem 5.3 that applies when the support of $f$ has small diameter, that is, that the Hoeffding projection

$$
h_{\varphi}^{+}(x):=\int_{\Sigma} h_{\varphi}(x, y) d \lambda(y)
$$

is not cohomologous to a scalar multiple of $F$. The first of these tasks will be carried out in Section 9.4 by an argument similar to that carried out in Section 7 above for the function $h$. The second will be addressed in Sections 9.2 and 9.3.

\subsection{Representation of the Hoeffding projection}

For each small $\delta>0$, define a function $H_{\delta}^{\varphi}: S \Upsilon \times S \Upsilon$ by setting $H_{\delta}^{\varphi}(u, v)=$ $\varphi(z(u, v))$ if the geodesic segments of length $\delta$ based at $u$ and $v$ intersect at a point $z(u, v) \in \Upsilon$, and by setting $H_{\delta}^{\varphi}(u, v)=0$ otherwise. This is the obvious analogue of the intersection kernel $H_{\delta}$ defined in Section 2. The primary difference between the self-intersection kernel $H_{\delta}$ and the localized kernel $H_{\delta}^{\varphi}$ is that the constant function 1 is not, in general, an eigenfunction of $H_{\delta}^{\varphi}$. To see this, define

$$
k_{\delta}^{\varphi}(u)=\frac{1}{\delta^{2} \kappa} \int_{S \Upsilon} H_{\delta}^{\varphi} v_{L}(d v),
$$

where $v_{L}$ is the normalized Liouville measure on $S \Upsilon$.

LEMMA 9.1

If $f: \Upsilon \rightarrow \mathbb{R}$ is continuous, then

$$
\lim _{\delta \rightarrow 0}\left\|k_{\delta}^{\varphi}-\varphi \circ p\right\|_{\infty}=0 .
$$

\section{Proof}

Because $\varphi$ is continuous and $H_{\delta}^{\varphi}(u, v)$ is nonzero only for pairs $u, v$ at distance less than $\delta$, the value of $\varphi(z(u, v))$ will be close to $\varphi(p u)$ when $\delta>0$ is small, uniformly for $u \in S \Upsilon$. Hence,

$$
\left|\varphi(p u) H_{\delta}(u, v)-\kappa \delta^{2} H_{\delta}^{\varphi}(u, v)\right| \leq \max _{d(u, v) \leq \delta}|\varphi(p u)-\varphi(p v)| .
$$


Since the constant function 1 is an eigenfunction of $H_{\delta}$, with eigenvalue $\delta^{2} \kappa$ (by Lemma 2.2), the result follows.

The relevance of the kernel $H_{\delta}^{\varphi}$ is that the Hoeffding projection $h_{\varphi}^{+}$defined by (71) can be expressed approximately in terms of $k_{\delta}^{\varphi}$. Both $h_{\varphi}^{+}$and $k_{\delta}^{\varphi}$ are defined as expectations of $\varphi$-values at intersection points of geodesic segments: (i) $h_{\varphi}^{+}(x)$ is the expected value of $f$ at the intersection point (if there is one) of the geodesic segments corresponding to the fibers $\mathcal{F}_{x}$ and $\mathcal{F}_{y}$ of the suspension flow when $y$ is chosen according to the distribution $\lambda$; and (ii) $k_{\delta}^{\varphi}$ is the corresponding expectation for the geodesic segments of fixed length $\delta$. Hence, for small $\delta$ the value of $h_{+}^{\varphi}(x)$ can be obtained approximately by integrating along the fiber $\mathcal{F}_{x}$. Together with Lemma 9.1, this implies that if $\gamma(t)$ is the orbit of the geodesic flow corresponding to the orbit $\phi_{t}(x, 0)$ of the suspension flow, then

$$
h_{+}^{\varphi}(x)=\lim _{\delta \rightarrow 0} \int_{0}^{F(x)-\delta} k_{\delta}(\gamma(s)) d s=\int_{0}^{F(x)} \varphi(p(\gamma(s))) d s .
$$

\subsection{Coboundaries of the geodesic flow}

If $f, g: \Sigma \rightarrow \mathbb{R}$ are Hölder-continuous functions, then a necessary and sufficient condition for $f$ and $g$ to be cohomologous is that they sum to the same values on all periodic sequences (see [11, Theorem 1.28] for the sufficiency). In particular, for every periodic sequence $x \in \Sigma$, if $x$ has period $n=n(x)$, then

$$
S_{n} f(x)=S_{n} g(x)
$$

In the case of interest, the relevant functions are integrals over fibers of the suspension space $\Sigma_{F}$. For the function $F$ this is obvious:

$$
F(x)=\int_{0}^{F(x)} 1 d s,
$$

while for the Hoeffding projection $h_{+}^{\varphi}$ it follows from formula (72). Consequently, for $F$ and $a h_{+}^{\varphi}$ to be cohomologous it is necessary that the function $a \varphi \circ p-1$ integrate to zero on every periodic orbit of the suspension flow. Since both $\varphi \circ p$ and the constant 1 are pullbacks of smooth functions on $\Upsilon$, this implies that $a \varphi-1$ must integrate to zero on every closed geodesic.

Call a continuous function $\psi: \Upsilon \rightarrow \mathbb{R}_{+}$a coboundary for the geodesic flow if it integrates to zero along every closed geodesic, and say that two functions are cohomologous if they differ by a coboundary. It is quite easy to construct a function $g: \Upsilon \rightarrow \mathbb{R}$ that is not cohomologous to a constant. Take two closed geodesics $\alpha$ and

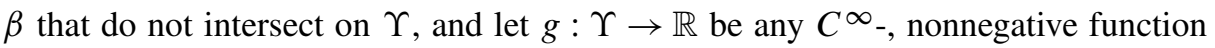
that is identically 1 along $\alpha$ but vanishes in a neighborhood of $\beta$; then by the criterion 
established above, $g$ cannot be cohomologous to a constant. In fact, the existence of nonintersecting closed geodesics yields the existence of a large class of functions that are not cohomologous to constants.

PROPOSITION 9.2

Let $\varepsilon>0$ be the distance in $\Upsilon$ between two nonintersecting closed geodesics $\alpha$ and $\beta$. Then no $C^{\infty}$-, nonnegative function $g: \Upsilon \rightarrow \mathbb{R}$ that is not identically zero and whose support has diameter less than $\varepsilon$ is cohomologous to a constant.

\section{Proof}

In order that $g$ be cohomologous to a constant $c$ it must be the case that the average value of $g$ along any closed geodesic is $c$. If $g$ is continuous and nonnegative, and not identically zero, then there is an open set $U$ in which $g$ is strictly positive. Because closed geodesics are dense in $S \Upsilon$, their projections are dense in $\Upsilon$, and so there is at least one closed geodesic $\xi$ that enters $U$. Since $g$ is continuous, its integral-and hence its average - along $\xi$ must be positive. However, by hypothesis, $g$ vanishes on at least one of the geodesics $\alpha, \beta$, and so there is at least one closed geodesic on which the average value of $g$ is zero.

\section{Remark 9.3}

That there exist pairs of nonintersecting closed geodesics on any negatively curved surface can be proved using the conformal equivalence of Riemannian metrics discussed in Section 3.2 above. First, elementary arguments in hyperbolic geometry show that there are nonintersecting closed geodesics on any surface of constant curvature -1 . Next, Proposition 3.3 implies that for any Riemannian metric $\varrho_{1}$ of variable negative curvature on a compact surface $\Upsilon$ there is a smooth deformation of $\varrho_{1}$ to a constant curvature metric $\varrho_{0}$ through metrics $\varrho_{s}$ of negative curvature. In this deformation, the closed geodesic in a given free homotopy class deforms smoothly; moreover, transversal intersections can be neither created nor destroyed. Therefore, if $\gamma_{0}, \gamma_{1}$ are nonintersecting closed geodesics relative to $\varrho_{0}$, then the closed geodesics $\gamma_{0}^{\prime}, \gamma_{1}^{\prime}$ in the corresponding free homotopy classes are also nonintersecting.

\subsection{Verification of Hypothesis 5.1}

It remains to show that the function $h_{\varphi}: \Sigma \times \Sigma \rightarrow \mathbb{R}_{+}$defined by (69) satisfies Hypothesis 5.1 relative to the measure $\lambda$, or to some equivalent (mutually a.c.) probability measure. For the same reason as in Section 7 (see in particular Lemma 7.2), we must allow for adjustment of the Poincare section of the suspension. Thus, for small $r \geq 0$, define

$$
h_{\varphi}^{r}(x, y)=\varphi\left(z_{r}(x, y)\right) h^{r}(x, y)
$$


where $h^{r}=1$ if the geodesic segments corresponding to the suspension flow segments $\mathscr{F}_{x}^{r}$ and $\mathscr{F}_{y}^{r}$ intersect at a point $z=z_{r}(x, y) \in \Upsilon$ and where $h^{r}=0$ if these segments do not intersect. Then the representation (70) holds with $h_{\varphi}$ replaced by $h_{\varphi}^{r}$, and the Hoeffding projection of $h_{\varphi}^{r}$ will again be given by (72), but with

$$
\int_{0}^{F(x)} \text { replaced by } \int_{r}^{F(x)+r} \text {. }
$$

For the verification of Hypothesis 5.1, we use the decomposition

$$
\begin{aligned}
& h_{\varphi}^{r}(x, y)=\sum_{m=1}^{\infty} \psi_{m}^{r}(x, y)+\psi_{\infty}^{r}(x, y), \quad \text { where } \\
& \quad \sum_{j=1}^{m} \psi_{j}^{r}(x, y)=\min \left\{h_{\varphi}^{r}\left(x^{\prime}, y^{\prime}\right): x_{i}^{\prime}=x_{i} \text { and } y_{i}^{\prime}=y_{i} \forall|i| \leq m\right\} .
\end{aligned}
$$

The functions $\psi_{m}^{r}$ are obviously symmetric, since $h_{\varphi}$ is, and $\psi_{m}^{r}$ depends only on the coordinates $|i| \leq m$. Moreover, each $\psi_{m}^{r}$ is nonnegative, and $\sum_{m} \psi_{m}^{r}+\psi_{\infty}^{r}=h_{\varphi}^{r}$ is bounded by $\|\varphi\|_{\infty}$. Hence, (H0), (H1), and (H2) of Hypothesis 5.1 all hold, leaving only (H3).

LEMMA 9.4

There exist constants $\varrho<\beta<1$ such that for all large $m$,

$$
\psi_{m}^{r}(x, y) \leq \beta^{m}
$$

unless the geodesic segments $p \circ \pi\left(\mathcal{F}_{x}^{r}\right)$ and $p \circ \pi\left(\mathcal{F}_{y}^{r}\right)$ intersect at angle less than $\varrho^{m}$ or at a point $z_{r}(x, y)$ within distance $\varrho^{m}$ of one of the endpoints of one of the geodesic segments.

\section{Proof}

For ease of exposition, we will discuss only the case $r=0$; the general case can be handled in the same manner. The semiconjugacy $\pi: \Sigma_{F} \rightarrow S \Upsilon$ is Hölder-continuous, so there exists $\alpha<1$ such that if two sequences $x, x^{\prime} \in \Sigma$ agree in coordinates $|i| \leq m$, then $\pi(x, s)$ and $\pi\left(x^{\prime}, s\right)$ are within distance $\alpha^{m}$ for all $s \in\left[0, F(x) \wedge F\left(x^{\prime}\right)\right]$, and $\left|F(x)-F\left(x^{\prime}\right)\right|<\alpha^{m}$, at least for sufficiently large $m$.

Suppose now that $x_{i}=x_{i}^{\prime}$ and $y_{i}=y_{i}^{\prime}$ for all $|i| \leq m$, and that the geodesic segments $p \circ \pi\left(\mathcal{F}_{x}\right)$ and $\mathcal{F}_{y}$ intersect at an angle not smaller than $\varrho^{m}$ and at a point $z(x, y)$ not within distance $\varrho^{m}$ of one of the endpoints. Then by (A3)' of Remark 3.8 (see Section 3.3), the geodesic segments $p \circ \pi\left(\mathcal{F}_{x^{\prime}}\right)$ and $\mathcal{F}_{y^{\prime}}$ will also intersect. Furthermore, if $\alpha<\varrho$ (as we may assume without loss of generality), then the intersection point $z\left(x^{\prime}, y^{\prime}\right)$ will lie within distance $\beta^{m}$ of $z(x, y)$, for some $\beta<1$. Since $\varphi$ is 
smooth, it follows that, for some $C<\infty$ depending on the $C_{1}$-norm of $\varphi$,

$$
\left|\varphi(z(x, y))-\varphi\left(z\left(x^{\prime}, y^{\prime}\right)\right)\right|<C \beta^{m} .
$$

Lemma 9.4 implies that to prove condition (H3) of Hypothesis 5.1, it suffices to establish the inequality (60). But this has already been done, in Lemmas 7.2-7.7. This yields the following result.

\section{PROPOSITION 9.5}

For any Gibbs state $\mu$, the functions $\psi_{m}^{r}$ satisfy (H3) of Hypothesis 5.1 relative to $\mu$ for almost every $r$ in some interval $\left[0, r_{*}\right]$ of positive length $r_{*}$.

\subsection{Proof of Theorem 1.2}

Proposition 9.5 implies that after appropriate modification of the Poincaré section of the suspension flow, the function $h_{\varphi}$ in the representation (70) of the localized selfintersection count meets the requirements of Corollary 5.5. Proposition 9.2 implies that if the support of $\varphi: \Upsilon \rightarrow \mathbb{R}_{+}$is less than the distance between two nonintersecting closed geodesics, and if $\varphi \geq 0$ is smooth and not identically zero, then the Hoeffding projection $h_{+}^{\varphi}$ of $h_{\varphi}$ relative to the measure $\lambda$ is not cohomologous to a constant multiple of $F$. By the argument of Section 9.1, it follows that the second case of Theorem 5.3 (see relation (47)) applies.

\section{0. $U$-statistics and randomly chosen periodic orbits}

The remainder of the article will be devoted to the proof of Theorem 1.3. The main technical tool, Theorem 10.2, will be an extension of Theorem 5.3 to measures concentrated on finite sets of periodic sequences. As in Theorem 5.3 there will be two cases, one leading to fluctuations of size $T$, the other to fluctuations of size $T^{3 / 2}$. Which of these two cases will apply will once again be determined by whether or not the relevant Hoeffding projection is cohomologous to a scalar multiple of the height function $F$ in the suspension flow. Proposition 6.3 gives necessary and sufficient conditions for this: the Hoeffding projection is cohomologous to a scalar multiple of $F$ if and only if the suspension measure $\mu^{*}$ associated with the Gibbs state $\mu$ is the pullback of the Liouville measure.

\subsection{An extension of Theorem 5.3}

It is well understood that the distribution of periodic orbits in a hyperbolic dynamical system is, in a certain sense, governed by the invariant measure of maximum entropy. There are two aspects of the connection. First, according to Margulis's prime geodesic theorem [26, Section 6, Theorem 5] (and its generalization in [30, Theorem 2]), the number of periodic orbits of minimal period less than $T$ grows like $e^{\theta T} /(\theta T)$, where 
$\theta$ is the entropy of the maximum entropy measure (see [25], [30]). Second, the empirical distribution of a random chosen periodic orbit (from among those with minimal period less than $T$ ) is, with high probability, close to the maximum entropy measure in the weak topology on measures (see [22, Theorem 7]). It is the latter connection that is primarily responsible for Theorem 1.3.

In this section, we will formulate and prove an extension of Theorem 5.3 for $U$ statistics of randomly chosen periodic orbits of a suspension flow. This result will be combined with the results of Section 3 on symbolic dynamics for geodesic flows to prove Theorem 1.3 in Section 11.

Fix a topologically mixing suspension flow on a suspension space $\Sigma_{F}$ over a shift $(\Sigma, \sigma)$ of finite type with a Hölder-continuous height function $F>0$. The invariant probability measure of maximum entropy for the suspension flow is the suspension $\mu_{-\theta F}^{*}$ (see Section 4.3) of the Gibbs state $\mu_{-\theta F}$, where $\theta$ is the unique positive number such that Pressure $(-\theta F)=0$. The value $\theta$ is the topological entropy of the suspension flow (see, e.g., [30], [22]).

Say that a sequence $x \in \Sigma$ represents an orbit $\gamma$ of the suspension flow if the point $(x, 0) \in \Sigma_{F}$ lies on the path $\gamma$. If $x \in \Sigma$ is a periodic sequence, then all of its cyclic shifts represent the same periodic orbit $p=p_{x}$ of the suspension flow, and these are the only representatives of $p$. Theorem 7 of [22] implies that for large $T$, nearly all of the periodic orbits of the suspension flow with minimal period approximately $T$ have the property that their representative periodic sequences have minimal period $T / E_{\mu_{-\theta F}} F+o(T)$. Hence, for large $T$ nearly all of the periodic orbits of the flow have $T / E_{\mu_{-\theta F}} F+o(T)$ representative sequences. This implies that the uniform distribution on periodic orbits of the flow with period approximately equal to $T$ is nearly identical to the image (under the natural correspondence) of the uniform distribution on periodic sequences $x$ such that $S_{\tau_{T}(x)} F(x) \approx T$. (Recall that $\tau(x)=\tau_{T}(x)$ is the smallest integer $n$ such that $S_{n} F(x) \geq T$, and $R_{T}(x)=S_{\tau(x)} F(x)-T$ is the overshoot.) Thus, we now change our focus from periodic orbits of the flow to periodic sequences.

A periodic sequence $x \in \Sigma$ represents a periodic orbit of the suspension flow with minimal period between $T$ and $T+\varepsilon$ if and only if (a) the period of the sequence $x$ is $\tau(x)$, and (b) $R_{T}(x)<\varepsilon$. Denote by $B_{T, \varepsilon}$ the set of all periodic sequences satisfying these conditions. This set is finite: in fact, Margulis's prime orbit theorem and the law of large numbers cited above (or, alternatively, [22, Theorem 1]) imply that

$$
\left|B_{T, \varepsilon}\right| \sim C e^{\theta T}\left(e^{\theta \varepsilon}-1\right) \quad \text { as } T \rightarrow \infty
$$

for a constant $C>0$ independent of $T$ and $\varepsilon$. Define

$$
\nu_{T, \varepsilon}=\text { uniform probability distribution on } B_{T, \varepsilon} \text {. }
$$


Our objective in this section is to extend the results of Theorem 5.3 to the family of measures $\nu_{T, \varepsilon}$. These results concern the large- $T$ limiting behavior of the distribution of the $U$-statistics $U_{T}$ defined by

$$
U_{T}(x):=\sum_{i=1}^{\tau(x)} \sum_{j=1}^{\tau(x)} h\left(\sigma^{i} x, \sigma^{j} x\right) .
$$

Since ultimately we will want to use these results to prove that the distribution of self-intersection counts of closed geodesics converges, it is important that they should hold for functions $h: \Sigma \times \Sigma \rightarrow \mathbb{R}$ that are not necessarily continuous. The following relatively weak hypothesis on the function $h$ is tailored to the particular case of selfintersection counts. For any periodic sequence $x$ with minimal period $\tau(x)=\tau_{T}(x)$ and any integer $m \geq 1$, define

$$
\Delta_{T}^{m} U(x)=\max \left|\sum_{i=1}^{\tau(x)} \sum_{j=1}^{\tau(x)}\left(h\left(\sigma^{i} x, \sigma^{j} x\right)-h\left(\sigma^{i} x^{\prime}, \sigma^{j} x^{\prime}\right)\right)\right|,
$$

where the maximum is over all sequences $x^{\prime} \in \Sigma$ (not necessarily periodic) such that $x_{i}^{\prime}=x_{i}$ for all $-m \leq i \leq \tau(x)+m$.

HYPOTHESIS 10.1

For each $\varepsilon>0$ there exist positive constants $\varepsilon_{m} \rightarrow 0$ as $m \rightarrow \infty$ such that for all sufficiently large $m \geq 1$ and $T$ (i.e., for $T \geq t_{m, \varepsilon}$ ),

$$
\nu_{T, \varepsilon}\left\{\Delta_{T}^{m} U(x) \geq \varepsilon_{m} T\right\}<\varepsilon_{m} .
$$

If $h$ is Hölder-continuous on $\Sigma \times \Sigma$, then Hypothesis 10.1 is trivially satisfied because in this case $\Delta_{T}^{m} U$ will be uniformly bounded by $\beta^{m} T$ for some $0<\beta<1$. In Section 11, we will show that the hypothesis holds for the function $h$ in the representation (25) of self-intersection counts.

\section{THEOREM 10.2}

Assume that $h: \Sigma \times \Sigma \rightarrow \mathbb{R}$ satisfies Hypothesis 10.1 and also Hypothesis 5.1 for the measure $\lambda=\mu_{-\theta F}$. Let $h_{+}$be the Hoeffding projection of $h$ relative to $\mu_{-\theta F}$ (see equation (71)), and let $\tilde{U}_{T}$ and $\tilde{\tau}_{T}$ be the renormalizations of $U_{T}$ and $\tau_{T}$ defined in (46), (47), and (45). (In particular, if $h_{+}$is cohomologous to a scalar multiple a $F$ of the height function $F$, then $\tilde{U}_{T}$ is defined by (46), but otherwise it is defined by (47).) Then as $T \rightarrow \infty$ the joint distribution of $\tilde{U}_{T}, \tilde{\tau}_{T}, R_{T}, x$, and $\sigma^{\tau_{T}}(x)(x)$ under $\nu_{T, \varepsilon}$ converges, and the limiting joint distribution of $\tilde{U}_{T}$ and $\tilde{\tau}_{T}$ is the same as under $\mu_{-\theta F}$ (i.e., by (46) and (47) of Theorem 5.3). 
The remainder of this section is devoted to the proof of this theorem. The strategy will be to deduce Theorem 10.2 from Corollary 5.6; however, since $v_{T, \varepsilon}$ is supported by a finite set of periodic sequences, it is mutually singular with respect to any Gibbs state, and so Corollary 5.6 does not apply directly. Consequently, we will be forced to show that $v_{T, \varepsilon}$ is close in the weak topology to a probability measure absolutely continuous relative to the Gibbs state $\mu_{-\theta F}$, to which Corollary 5.6 does apply. Hypothesis 10.1 will ensure that the distribution of $\tilde{U}_{T}$ under this approximating measure is close to its distribution under $v_{T, \varepsilon}$, and so Theorem 10.2 will follow.

Observe that to prove Theorem 10.2 it suffices to prove the result for small values of $\varepsilon>0$, because for each $T>0$ and each integer $M \geq 1$ the measure $\nu_{T, \varepsilon}$ is a convex combination of the measures $v_{T+i \varepsilon / M, \varepsilon / M}$.

\subsection{Skeleton of the proof}

To show that $v_{T, \varepsilon}$ is weakly close to a probability measure absolutely continuous with respect to $\mu_{-\theta F}$, we will partition the support $B_{T, \varepsilon}$ of $v_{T, \varepsilon}$ into subsets on which the "likelihood function" of the measure $\mu_{-\theta F}$ (see equation (32)) is nearly constant. (See Proposition 10.3 below for a precise statement.) This will imply that the uniform distribution on each set $A$ of the partition is weakly close to the normalized restriction of $\mu_{-\theta F}$ to the cylinder set of all sequences in $\Sigma$ that agree with some element $x \in A$ in coordinates $-m \leq j \leq \tau(x)+j$, for some large $m$.

To define the partition, for each $x \in \Sigma$ and $m \geq 1$, let $B_{T, \varepsilon, m, x}$ be the set of all $y \in B_{T, \varepsilon}$ such that

$$
y_{j}=y_{\tau(y)+j}=x_{j} \quad \text { for all } j \in[-m, m] .
$$

Note that since $y \in B_{T, \varepsilon}$ it is periodic with period $\tau(y)=\tau_{T}(y)$, so the restriction $y_{\tau(y)+j}=x_{j}$ is redundant. Define $\nu_{T, \varepsilon, m, x}$ to be the uniform distribution on $B_{T, \varepsilon, m, x}$. Clearly, $B_{T, \varepsilon, m, x}$ depends on $x$ only by way of the coordinates $x_{[-m, m]}$, and the sets $B_{T, \varepsilon, m, x}$ are pairwise disjoint, so they partition $B_{T, \varepsilon}$. Thus, for each $m \geq 1$,

$$
v_{T, \varepsilon}=\sum_{x_{[-m, m]}} \frac{\left|B_{T, \varepsilon, m, x}\right|}{\left|B_{T, \varepsilon}\right|} v_{T, \varepsilon, m, x},
$$

where the sum is over all admissible sequences $x_{[-m, m]}$, that is, sequences obtained by restricting sequences $x \in \Sigma$. Theorem 1 of [22] implies that for each $x \in \Sigma$ and $m \geq 1$, as $T \rightarrow \infty$,

$$
\left|B_{T, \varepsilon, m, x}\right| \sim \mu_{-\theta F}\left(\Sigma_{[-m, m]}(x)\right)\left|B_{T, \varepsilon}\right| .
$$

Since $\mu\left(\Sigma_{[-m, m]}(x)\right)>0$ for any admissible $x_{[-m, m]}$, this estimate implies that for each $x_{[-m, m]}$ the set $\left|B_{T, \varepsilon, m, x}\right|$ grows exponentially with $T$. In particular, for all sufficiently large $T$ the set $B_{T, \varepsilon, m, x}$ is nonempty, and so $v_{T, \varepsilon, m, x}$ is well defined. 
PROPOSITION 10.3

There exist constants $C=C_{\varepsilon}, t_{m}<\infty$ and $\beta \in(0,1)$ such that for all sufficiently large $m$, for all $T \geq t_{m}$, and for any two periodic sequences $x, y \in B_{T, \varepsilon}$ for which (80) holds,

$$
1-C \beta^{m} \leq \frac{\mu_{-\theta F}\left(\Sigma_{[-m, \tau(x)+m]}(x)\right)}{\mu_{-\theta F}\left(\Sigma_{[-m, \tau(y)+m]}(y)\right)} \frac{e^{-\theta S_{\tau(y)} F(y)}}{e^{-\theta S_{\tau(x)} F(x)}} \leq 1+C \beta^{m} .
$$

Since $\operatorname{Pr}(-\theta F)=0$, it follows from the definition (32) of a Gibbs state that the ratio in (83) is bounded above and below. The proof that the upper and lower bounds are within $C \beta^{m}$ of 1 will rely on the periodicity of the sequences $x, y$ and the condition (80), together with the spectral theory of the Ruelle operator. The details of the argument are deferred to Section 10.4 below.

\section{Remark 10.4}

It is not assumed in Proposition 10.3 that $\tau(x)=\tau(y)$, so the number of coordinates specified in the two cylinder sets appearing in (83) need not be the same. Also, by definition of $\tau=\tau_{T}$, the sums $S_{\tau(x)} F(x)$ and $S_{\tau(y)} F(y)$ both lie in the interval $[T, T+\varepsilon]$, so the ratio of exponentials in (83) is bounded above and below by $e^{ \pm \theta \varepsilon}$. Thus, for small $\varepsilon$ the measures of the cylinder sets in (83) are nearly equal.

For any integer $m \geq 1$, define $\operatorname{Var}_{m} F$ to be the maximum difference $\mid F(x)-$ $F(y) \mid$ for sequences $x, y \in \Sigma$ such that $x_{j}=y_{j}$ for all $|j| \leq m$. Because the function $F$ is Hölder-continuous, the sequence $\operatorname{Var}_{m} F$ decays exponentially in $m$. Consequently, if two sequences $x, y \in \Sigma$ satisfy $x_{j}=y_{j}$ for all $-m \leq j \leq n+m$, then

$$
\left|S_{n} F(x)-S_{n} F(y)\right|<\delta_{m}:=3 \sum_{k=m}^{\infty} \operatorname{Var}_{k} F
$$

The sequence $\delta_{m}$ decays exponentially with $m$.

Assume henceforth that $\varepsilon<\min F$. This guarantees that if $T<S_{n} F(x) \leq T+\varepsilon$, then $\tau(x)=n$. Assume also that $m$ is large enough that $5 \delta_{m}<\varepsilon$. For each $m \geq 1$ and $x \in \Sigma$, define $A_{T, \varepsilon, m, x}$ to be the set of all sequences $y \in \Sigma$ that satisfy (80) and are such that $0<R_{T}(y) \leq \varepsilon$, and define $\lambda_{T, \varepsilon, m, x}$ to be the probability measure with support $A_{T, \varepsilon, m, x}$ that is absolutely continuous relative to $\mu_{-\theta F}$ with Radon-Nikodym derivative

$$
\frac{d \lambda_{T, \varepsilon, m, x}}{d \mu_{-\theta F}}(z)=C e^{\theta R_{T}(z)} I_{A_{T, \varepsilon, m}(x)}(z),
$$

where $C=C_{T, \varepsilon, m, x}$ is the normalizing constant needed to make $\lambda_{T, \varepsilon, m, x}$ a probability measure. In Section 10.3 below we will show that when $m$ and $T$ are large, the set 
$A_{T, \varepsilon, m, x}$ nearly coincides with

$$
A_{T, \varepsilon, m, x}^{*}:=\bigcup_{y \in B_{T, \varepsilon, m, x}} \Sigma_{[-m, \tau(y)+m]}(y),
$$

and so Proposition 10.3 will imply that the probability measure $\lambda_{T, \varepsilon, m, x}$ distributes its mass nearly uniformly over the cylinder sets in the union $A_{T, \varepsilon, m, x}^{*}$. Using this, we will prove that, for large $m$, the measure $\lambda_{T, \varepsilon, m, x}$ is close in the weak (Lévy) topology to $v_{T, \varepsilon, m, x}$. It will be most convenient to formulate this statement using the following coupling metric for the weak topology on the space of Borel probability measures. (See [40] or [17] for a proof that the coupling metric generates the weak topology.)

\section{Definition 10.5}

Let $Q_{A}, Q_{B}$ be Borel probability measures on a complete, separable metric space $(\mathcal{X}, d)$. The coupling distance $d_{C}\left(Q_{A}, Q_{B}\right)$ between $Q_{A}$ and $Q_{B}$ is the infimal $\kappa \geq 0$ for which there exists a Borel probability measure $Q$ on $X \times \mathcal{X}$ with marginals $Q_{A}$ and $Q_{B}$ such that

$$
Q\{(x, y): d(x, y)>\kappa\}<\kappa \text {. }
$$

Since this definition requires a metric on $\mathcal{X}$, we must specify a metric for the case $\mathcal{X}=\Sigma$, so henceforth we will let $d=d_{\Sigma}$ be the metric $d(x, y)=2^{-n(x, y)}$, where $n(x, y)$ is the minimum nonnegative integer $n$ such that $x_{j} \neq y_{j}$ for either $j=n$ or $j=-n$.

PROPOSITION 10.6

There exist constants $C=C_{\varepsilon}, t_{m}<\infty$ and $0<\beta<1$ such that, for all sufficiently large $m$ and $T \geq t_{m}$ and for all $x \in \Sigma$,

$$
d_{C}\left(v_{T, \varepsilon, m, x}, \lambda_{T, \varepsilon, m, x}\right) \leq C e^{\theta e} \beta^{m} .
$$

Consequently, for all $\varepsilon>0$ and for all sufficiently large $m$ and $T$,

$$
d_{C}\left(v_{T, \varepsilon}, \lambda_{T, \varepsilon, m}\right) \leq 2 C \beta^{m},
$$

where

$$
\lambda_{T, \varepsilon, m}:=\sum_{x-m, m} \mu_{-\theta F}\left(\Sigma_{[-m, m]}(x)\right) \nu_{T, \varepsilon, m, x}
$$

The proof is given in Section 10.3 below. 
LEMMA 10.7

Fix $\varepsilon>0$ and $m \geq 1$ large enough that $5 \delta_{m}<\varepsilon$. Then for each $x \in \Sigma$ the conclusions of Corollary 5.6 hold for the family of probability measures $\left(\lambda_{T, \varepsilon, m, x}\right)_{T \geq 1}$. Consequently, they hold also for the family $\left\{\lambda_{T, \varepsilon, m}\right\}_{T \geq 1}$.

\section{Proof}

We must show that the Radon-Nikodym derivatives in equation (85) have the form (53). By definition, the set $A_{T, \varepsilon, m, x}$ consists of all sequences $z \in \Sigma$ such that $R_{T}(z) \in$ $(0, \varepsilon]$ and such that (80) holds (with $y=z)$. Hence, the likelihood ratio (85) can be factored as

$$
e^{-\theta R_{T}(z)} I_{A_{T, \varepsilon, m}(x)}(z)=e^{-\theta R_{T}(z)} I_{(0, \varepsilon]}\left(R_{T}(z)\right) I_{\Sigma_{-m, m}(x)}(z) I_{\Sigma_{-m, m}(x)}\left(\sigma^{\tau(z)} z\right) .
$$

This is clearly of the form (53). Although the function $g_{3}$ in this factorization is not continuous (because of the indicator $\left.I_{(0, \varepsilon]}\right)$, it is piecewise continuous and bounded, so by Remark 5.7 the conclusions of Corollary 5.6 are valid for the family $\left(\lambda_{T, \varepsilon, m, x}\right)_{T \geq 1}$. Since each of the probability measures $\lambda_{T, \varepsilon, m}$ is a convex combination of the measures $\lambda_{T, \varepsilon, m, x}$ (see equation (89)), it follows that the conclusions of Corollary 5.6 hold also for the family $\left\{\lambda_{T, \varepsilon, m}\right\}_{T \geq 1}$.

\section{Proof of Theorem 10.2}

Given Proposition 10.6 and Lemma 10.7, Theorem 10.2 follows routinely. Lemma 10.7 implies that for all sufficiently large $m$ the conclusions of Theorem 10.2 hold if the measures $\nu_{T, \varepsilon}$ are replaced by $\lambda_{T, \varepsilon, m}$. Thus, to complete the proof, it will suffice to show that, for any $\delta>0$ and any continuous, bounded function $\varphi: \mathbb{R}^{3} \times \Sigma^{2} \rightarrow \mathbb{R}$, there exists $m$ sufficiently large such that

$$
\underset{T \rightarrow \infty}{\limsup }\left|E_{\lambda_{T, \varepsilon, m}} \Phi-E_{v_{T, \varepsilon}} \Phi\right| \leq \delta \quad \text { where } \Phi(x)=\varphi\left(\tilde{U}_{T}, \tilde{\tau}_{T}, R_{T}, x, \sigma^{\tau(x)} x\right) .
$$

By Proposition 10.6, for all sufficiently large $m$ and $T$ there exist Borel probability measures $Q=Q_{T, \varepsilon, m}$ on $\Sigma^{2}$ with marginals $\nu_{T, \varepsilon}$ and $\lambda_{T, \varepsilon, m}$ such that (86) holds with $\kappa=2 C e^{\theta \varepsilon} \beta^{m}$. Now for any probability measure $Q$ on $\Sigma \times \Sigma$ with marginals $\nu_{T, \varepsilon}$ and $\lambda_{T, \varepsilon, m}$,

$$
E_{\lambda_{T, \varepsilon, m}} \Phi-E_{v_{T, \varepsilon}} \Phi=\int_{\Sigma \times \Sigma}(\Phi(x)-\Phi(y)) d Q(x, y) .
$$

By (86),

$$
\int_{d(x, y)>\kappa_{m}}|\Phi(x)-\Phi(y)| d Q(x, y) \leq \kappa_{m}\|\Phi\|_{\infty},
$$

where $\kappa_{m}=2 C e^{\theta \varepsilon} \beta^{m}$. By choosing $m$ sufficiently large, we may arrange that $\kappa_{m}\|\Phi\|_{\infty}<\delta / 2$. Thus, to prove (90) we must bound the integral of $|\Phi(x)-\Phi(y)|$ on 
the set of pairs $(x, y)$ such that $d(x, y) \leq \kappa_{m}$. This is where Hypothesis 10.1 will be used.

None of the functions $U_{T}(x), \tau_{T}(x)$, nor $R_{T}(x)$ is continuous in $x$. However, if $m$ is so large that $5 \delta_{m}<\varepsilon$, then inequality (84) implies that if $d(x, y)<2^{-m}$, then $\tau(x)=\tau(y)$ and $\left|R_{T}(x)-R_{T}(y)\right|<\delta_{m}$ unless

$$
R_{T}(z) \in\left[0, \delta_{m}\right] \cup\left[\varepsilon-\delta_{m}\right] \quad \text { for either } z=x \text { or } z=y .
$$

Since $Q$ has marginals $\nu_{T, \varepsilon}$ and $\lambda_{T, \varepsilon, m}$, the estimates (76) (for the first marginal $\nu_{T, \varepsilon}$ ) and (40) (for the second marginal $\lambda_{T, \varepsilon, m}$, using the fact that this measure is absolutely continuous relative to $\mu_{-\theta F}$ ) imply that for each $\varepsilon>0$ there exist constants $C_{\varepsilon}<\infty$ and $t_{m}<\infty$ such that, if $m$ is large enough that $5 \delta_{m}<\varepsilon$ and $T \geq t_{m}$, then

$$
\begin{aligned}
& Q\left\{(x, y): R_{T}(x) \in\left[0, \delta_{m}\right] \cup\left[\varepsilon-\delta_{m}\right]\right\}<C_{\varepsilon} \delta_{m} \text { and } \\
& Q\left\{(x, y): R_{T}(y) \in\left[0, \delta_{m}\right] \cup\left[\varepsilon-\delta_{m}\right]\right\}<C_{\varepsilon} \delta_{m} .
\end{aligned}
$$

Now if $\tau(x)=\tau(y)$, then, depending on whether or not $h_{+}$is cohomologous to a scalar multiple of the height function $F$ (see equations (46), (47)),

$$
\tilde{U}_{T}(x)-\tilde{U}_{T}(y)=T^{-\alpha} \sum_{i=1}^{\tau(x)} \sum_{j=1}^{\tau(x)}\left(h\left(\sigma^{i} x, \sigma^{j} x\right)-h\left(\sigma^{i} y, \sigma^{j} y\right)\right)
$$

for either $\alpha=1$ or $\alpha=3 / 2$. In either case, Hypothesis 10.1 ensures that there exist positive constants $\varepsilon_{m} \rightarrow 0$ such that, for all large $m$ and $T$,

$$
Q\left\{(x, y): \tau(x)=\tau(y) \text { and }\left|\tilde{U}_{T}(x)-\tilde{U}_{T}(y)\right|>\varepsilon_{m}\right\}<\varepsilon_{m} .
$$

Since the sequences $\varepsilon_{m}, \delta_{m}$, and $\kappa_{m}$ all converge to zero as $m \rightarrow \infty$, it now follows from the continuity of $\varphi$ that for sufficiently large $m$ and $T$,

$$
\int_{d(x, y) \leq \kappa_{m}}|\Phi(x)-\Phi(y)| d Q(x, y) \leq \delta / 2 .
$$

\subsection{Proof of Proposition 10.6}

In this section we show how Proposition 10.6 follows from Proposition 10.3. The proof of Proposition 10.3 is given in Section 10.4 below.

Fix $\varepsilon \in(0, \min F)$, and let $m$ be sufficiently large that $5 \delta_{m}<\varepsilon$. For each $x \in B_{T, \varepsilon}$, define

$$
A_{T, \varepsilon, m, x}^{*}=\bigcup_{y \in B_{T, \varepsilon, m, x}} \Sigma_{[-m, \tau(y)+m]}(y) .
$$


Since $B_{T, \varepsilon, m, x}$ depends only on the finite subsequence $x_{[-m, m]}$, the same is true of the set $A_{T, \varepsilon, m, x}^{*}$. The estimate (82) implies that for large $T$ there will be many representative sequences $x^{\prime} \in B_{T, \varepsilon, m, x}$ for which $2 \delta_{m}<R_{T}\left(x^{\prime}\right)<\varepsilon-4 \delta_{m}$; assume henceforth that $x$ is such a sequence, that is, that $x \in B_{T+2 \delta_{m}, \varepsilon-4 \delta_{m}}$. Then by (84), for any $y \in B_{T, \varepsilon, m, x}$ it must be the case that $\tau(y)=\tau(x)$; hence, the cylinder sets in the union (91) are pairwise disjoint. Therefore, there is a well-defined mapping $z \mapsto \hat{z}$ from $A_{T, \varepsilon, m, x}^{*}$ to $B_{T, \varepsilon, m, x}$ that sends each $z$ to the element $y \in B_{T, \varepsilon, m, x}$ that indexes the cylinder set of the partition (91) which contains $z$. This mapping has the property that no point $z$ is moved a distance more than $2^{-m}$ (in the usual metric $d=d_{\Sigma}$ on $\Sigma$ ); in fact,

$$
d_{\Sigma}\left(\sigma^{i} z, \sigma^{i} \hat{z}\right) \leq 2^{-m} \text { for all }-m \leq i \leq \tau(z)+m .
$$

For each $x \in B_{T+\delta_{m}, \varepsilon-\delta_{m}}$ define $\lambda_{T, \varepsilon, m, x}^{*}$ to be the probability measure on $A_{T, \varepsilon, m, x}^{*}$ that is absolutely continuous with respect to $\mu_{-\theta F}$ with Radon-Nikodym derivative

$$
\frac{d \lambda_{T, \varepsilon, m, x}^{*}}{d \mu_{-\theta F}}(z)=C_{T, \varepsilon, m, x}^{*} e^{-\theta R_{T}(\hat{z})} I_{A_{T, \varepsilon, m, x}^{*}}(z),
$$

where $C^{*}(T, \varepsilon, m, x)$ is the normalizing constant needed to make $\lambda_{T, \varepsilon, m, x}^{*}$ a probability measure. The Radon-Nikodym derivative is constant on each of the cylinder sets in the partition (91), and its values on these cylinder sets are chosen so as to cancel the exponential factors in (83). Consequently, by Proposition 10.3, if $y, z \in B_{T, \varepsilon, m}(x)$, then for constants $C_{\varepsilon}<\infty$ depending only on $\varepsilon$,

$$
1-C_{\varepsilon} \beta^{m} \leq \frac{\lambda_{T, \varepsilon, m, x}^{*}\left(\Sigma_{[-m, \tau(x)+m]}(z)\right)}{\lambda_{T, \varepsilon, m, x}^{*}\left(\Sigma_{[-m, \tau(y)+m]}(y)\right)} \leq 1+C_{\varepsilon} \beta^{m} .
$$

COROLLARY 10.8

Assume that $x \in B_{T+2 \delta_{m}, \varepsilon-2 \delta_{m}}$. Let $\lambda_{T, \varepsilon, m, x}^{\dagger}$ be the pushforward of the probability measure $\lambda_{T, \varepsilon, m, x}^{*}$ under the mapping $z \mapsto \hat{z}$ (i.e., the distribution of $\hat{z}$ when $z$ has distribution $\left.\lambda_{T, \varepsilon, m, x}^{*}\right)$. Then for suitable constants $C=C_{\varepsilon}<\infty$ and $\beta \in(0,1)$ not depending on $T, m$, or $x$,

$$
\left|\frac{d \lambda_{T, \varepsilon, m, x}^{\dagger}}{d \nu_{T, \varepsilon, m, x}}-1\right| \leq C \beta^{m}
$$

and consequently,

$$
d_{C}\left(\lambda_{T, \varepsilon, m, x}^{\dagger}, v_{T, \varepsilon, m, x}\right) \leq 1-\left(1-C \beta^{m}\right)^{-1}
$$

Proof

The first inequality is a direct consequence of (94). The second follows from the first 
by the following elementary fact: if $\nu, \mu$ are mutually absolutely continuous probability measures whose Radon-Nikodym derivatives $d \nu / d \mu$ and $d \mu / d \nu$ are both bounded below by $\varrho \in(0,1]$, then their coupling distance is no greater than $1-\varrho$.

Since the mapping $z \mapsto \hat{z}$ moves each $z$ by a distance at most $2^{-m}$, coupling distance between the probability measures $\lambda_{T, \varepsilon, m, x}^{\dagger}$ and $\lambda_{T, \varepsilon, m, x}^{*}$ is at most $2^{-m}$. By Corollary 10.8, the coupling distance between $\lambda_{T, \varepsilon, m, x}^{\dagger}$ and $v_{T, \varepsilon, m, x}$ is at most $C^{\prime} \beta^{m}$ for a suitable $C^{\prime}=C_{\varepsilon}^{\prime}<\infty$. Therefore, to prove Proposition 10.6 it suffices to prove the following lemma.

LEMMA 10.9

For each $\varepsilon>0$ there exists a constant $C_{\varepsilon}<\infty$ such that, for all $x \in \Sigma$, all $m$ large enough that $\delta_{m}<5 \varepsilon$, and all large $T$,

$$
d_{C}\left(\lambda_{T, \varepsilon, m, x}^{*}, \lambda_{T, \varepsilon, m, x}\right) \leq C_{\varepsilon} \delta_{m}
$$

\section{Proof}

Recall that without loss of generality we may assume (for $T$ large) that the representative sequence $x \in \Sigma$ is an element of $B_{T+2 \delta_{m}, \varepsilon-2 \delta_{m}}$. It must then be the case, by inequality (84), that every $y \in B_{T, \varepsilon, m, x}$ must have period $\tau(y)=\tau(x)$, and that $R_{T}(y) \in\left(\delta_{m}, \varepsilon-\delta_{m}\right)$. This in turn implies that every $z$ in the cylinder $\Sigma_{[-m, \tau(y)+m]}(y)$ must also satisfy $\tau(z)=\tau(x)$ and $R_{T}(z) \in(0, \varepsilon)$. Consequently, the support sets of the measures $\lambda_{T, \varepsilon, m, x}^{*}$ and $\lambda_{T, \varepsilon, m, x}$ satisfy

$$
A_{T, \varepsilon, m, x}^{*} \subset A_{T, \varepsilon, m, x} .
$$

Next, suppose that $z \in A_{T, \varepsilon, m, x}$ is such that $R_{T}(z) \in\left(\delta_{m}, \varepsilon-\delta_{m}\right)$. Let $\tilde{z}$ be the periodic sequence with period $\tau(z)$ that agrees with $z$ in coordinates $j \in[-m, \tau(z)+$ $m]$; then by the same argument as above, using inequality (84), we have $R_{T}(\tilde{z}) \in$ $(0, \varepsilon)$, and so $\tilde{z} \in B_{T, \varepsilon, m, x}$. By construction, $z$ is in the cylinder $\Sigma_{[-m, \tau(z)+m]}(\tilde{z})$, so it follows that $z \in A_{T, \varepsilon, m, x}^{*}$ and $\tilde{z}=\hat{z}$. This proves that

$$
A_{T, \varepsilon, m}(x) \backslash A_{T, \varepsilon, m, x}^{*} \subset\left\{z: R_{T}(z) \notin\left(\delta_{m}, \varepsilon-\delta_{m}\right)\right\} .
$$

These arguments also show that for every $z \in A_{T, \varepsilon, m, x}$ such that $R_{T}(z) \in\left(\delta_{m}, \varepsilon-\right.$ $\left.\delta_{m}\right)$, and for every $z \in A_{T, \varepsilon, m, x}^{*}$,

$$
\left|\frac{e^{-\theta R_{T}(z)}}{e^{-\theta R_{T}(\hat{z})}}-1\right|<e^{\theta \delta_{m}}-1 .
$$

Relations (98), (99), and (100) imply that the ratio of the Radon-Nikodym derivatives (93) and (85) differs from 1 by less than $C \delta_{m}$ except on the set 


$$
A_{T, \varepsilon, m, x}^{* *}:=\left\{z \in A_{T, \varepsilon, m, x}: R_{T}(z) \notin\left(\delta_{m}, \varepsilon-\delta_{m}\right)\right\} .
$$

But the renewal theorem (see inequality (40)) implies that, for some constant $C_{\varepsilon}^{\prime}$ independent of $m$, for all large $T$,

$$
\frac{\mu_{-\theta F}\left(A_{T, \varepsilon, m, x}^{* *}\right)}{\mu_{-\theta F}\left(A_{T, \varepsilon, m, x}\right)} \leq C_{\varepsilon}^{\prime} \delta_{m} .
$$

It now follows by routine arguments that for a suitable constant $C_{\varepsilon}^{\prime \prime}$, the total variation distance between the measures $\lambda_{T, \varepsilon, m, x}^{*}$ and $\lambda_{T, \varepsilon, m, x}$ is bounded above by $C_{\varepsilon}^{\prime \prime} \delta_{m}$ for large $m$ and large $T$. This implies (97).

\subsection{Proof of Proposition 10.3}

Recall that any Hölder-continuous function on $\Sigma$ is cohomologous to a Höldercontinuous function that depends only on the forward coordinates. Let $F$ be the height function of the suspension, and let $F_{+}$be a function of the forward coordinates that is cohomologous to $F$. Then $-\theta F$ and $-\theta F_{+}$have the same topological pressure (which by choice of $\theta$ is 0 ), and the Gibbs states $\mu_{-\theta F}$ and $\mu_{-\theta F_{+}}$are identical. Also, for any periodic sequence $x \in \Sigma$ with period (say) $n$, it must be the case that $S_{n} F(x)=S_{n} F_{+}(x)$. Since the assertion (83) involves only periodic sequences and measures of events under $\mu_{-\theta F}$, to prove (83) it will suffice to prove (83) with $F$ replaced by $F_{+}$. Thus, the representation (36) for the Gibbs state $\mu_{-\theta F_{+}}$can be used; in particular, since $\operatorname{Pr}\left(-\theta F_{+}\right)=0$,

$$
\frac{d \mu_{-\theta F_{+}}}{d_{v_{-\theta F_{+}}}}=h_{-\theta F_{+}},
$$

where $v$ and $h$ are the right and left eigenvectors of the Ruelle operator $\mathscr{L}=\mathscr{L}_{-} \theta F_{+}$. (For the remainder of the proof we will drop the subscripts on $h, v, \mu$, and $\mathscr{L}$.)

The measure $\mu$ is shift-invariant, so the cylinder sets in (83) can be shifted by $\sigma^{-m}$, and hence can be regarded as cylinder sets in the one-sided sequence space $\Sigma^{+}$. Fix a periodic sequence $x$ of period $n>2 m+1$; then

$$
\begin{aligned}
\mu\left(\Sigma_{[0, n+2 m]}(x)\right) & =\int_{\Sigma_{[0, n+2 m]}^{+}(x)} h d v \\
& =\int_{\Sigma_{[0, n+2 m]}(x)} h d\left(\left(\mathscr{L}^{*}\right)^{n+m} v\right) \\
& =\int_{\Sigma^{+}}\left(\mathscr{L}^{n+m}\left(h I_{\Sigma_{[0, n+2 m]}^{+}(x)}\right)\right) d \nu .
\end{aligned}
$$

Here we have used the fact that $v$ is an eigenmeasure of the adjoint $\mathscr{L}^{*}$ of the Ruelle operator with eigenvalue 1 . Next we use the definition of the Ruelle operator (see [11, 
Chapter 1, Section B]) to write, for any $z \in \Sigma^{+}$,

$$
\mathscr{L}^{n+m}\left(h I_{\Sigma_{[0, n+2 m]}^{+}(x)}\right)(z)=\sum_{y \in \Sigma^{+}: \sigma^{n+m} y=z} e^{-\theta S_{n+m} F(y)} h(y) I_{\Sigma_{[0, n+2 m]}^{+}(x)}(y) .
$$

The indicator function in this expression guarantees that the only $z \in \Sigma^{+}$for which there is a nonzero term in the sum are those sequences such that $z_{j}=x_{j}$ for $j \in$ $[0,2 m]$. (Keep in mind that $x$ is periodic with period $n$.) For each such $z$ there is exactly one $y \in \Sigma^{+}$for which the summand is nonvanishing, to wit, the sequence $(x \mid z)_{2 m+1}:=x_{0} x_{1} \cdots x_{2 m} z$ obtained by prefixing to $z$ the first $2 m+1$ letters of $x$. Consequently,

$$
\begin{aligned}
\mu\left(\Sigma_{[0, n+2 m]}(x)\right) & =\int_{\Sigma_{2 m+1}^{+}(x)} \exp \left\{-\theta S_{n+m} F\left((x \mid z)_{2 m+1}\right)\right\} h\left((x \mid z)_{2 m+1}\right) d v(z) \\
& =e^{-\theta S_{n+m} F(x)} \mu\left(\Sigma_{[0,2 m]}(x)\right)\left(1 \pm C \beta^{m}\right)
\end{aligned}
$$

for suitable constants $C<\infty$ and $0<\beta<1$ independent of $x$. The final approximate equality follows from the Hölder continuity of $F$ and $h$, together with inequality (84). It now follows that if $x, x^{\prime}$ are any periodic sequences with periods $n=\tau\left(\sigma^{m} x\right)>$ $2 m+1$ and $n^{\prime}=\tau\left(\sigma^{m} x^{\prime}\right)>2 m+1$ such that $x_{[0,2 m]}=x_{[0,2 m]}^{\prime}$, then

$$
\begin{aligned}
\frac{\mu\left(\Sigma_{[0, n+2 m]}(x)\right)}{\mu\left(\Sigma_{\left[0, n^{\prime}+2 m\right]}\left(x^{\prime}\right)\right)} & =\frac{e^{-\theta S_{m+n} F(x)}}{e^{-\theta S_{m+n^{\prime}} F\left(x^{\prime}\right)}}\left(1 \pm C^{\prime} \beta^{m}\right) \\
& =\frac{e^{-\theta S_{n} F\left(\sigma^{m} x\right)}}{e^{-\theta S_{n^{\prime}} F\left(\sigma^{m} x^{\prime}\right)}}\left(1 \pm C^{\prime \prime} \beta^{m}\right)
\end{aligned}
$$

for suitable $C^{\prime}, C^{\prime \prime}<\infty$. This implies relation (83).

\section{Proof of Theorem 1.3}

The results of Section 3 imply that, for any compact surface $\Upsilon$ equipped with a smooth Riemannian metric of negative curvature, the geodesic flow on $S \Upsilon$ is semiconjugate (by a Hölder-continuous mapping) to a suspension flow $\left(\Sigma_{F}, \phi_{t}\right)$ over a shift of finite type. All but finitely many closed geodesics correspond uniquely to periodic orbits of this suspension flow, and for each of these the self-intersection count is given by equation (25), or by equation (25) with $h$ replaced by $h^{r}$, for some small $r \geq 0$. By Proposition 7.1, there exist values of $r$ such that the function $h^{r}$ satisfies the hypotheses of Theorem 5.3 relative to any Gibbs state; for simplicity we will assume that the Poincaré section of the suspension has been adjusted so that $r=0$. If the Riemannian metric on $\Upsilon$ has constant curvature, then the normalized Liouville measure for the geodesic flow coincides with the maximum entropy-invariant measure, and so in this case Proposition 6.3 implies that the Hoeffding projection of $h$ relative to the 
Gibbs state $\lambda=\mu_{-\theta F}$ is a scalar multiple of $F$. On the other hand, if the Riemannian metric has variable negative curvature, then the maximum entropy measure is singular relative to Liouville measure, and so in this case, by Proposition 6.3, the Hoeffding projection of $h$ relative to the Gibbs state $\lambda=\mu_{-\theta F}$ is not cohomologous to a scalar multiple of $F$. Therefore, in either case, Theorem 1.3 will follow from Theorem 10.2, provided that Hypothesis 10.1 can be verified. This we will accomplish by reducing the problem to a problem about crossing rates.

The following lemma asserts that, for compact surfaces of constant negative curvature, the ergodic law (55) for intersections with a fixed geodesic segment extends from random geodesics to closed geodesics. Denote by $\lambda_{T, \varepsilon}$ the uniform distribution on the set of all (prime) closed geodesics with length in $[T, T+\varepsilon]$, and let $\kappa \Upsilon=1 / 4 \pi|\Upsilon|$. For any geodesic arc $\alpha$, let $|\alpha|$ be the length of $\alpha$.

\section{LEMMA 11.1}

Assume that $\Upsilon$ is a negatively curved compact surface. For any geodesic segment $\alpha$ and any closed geodesic $\beta$, let $N(\alpha ; \beta)$ be the number of transversal intersections of $\beta$ with $\alpha$. Then there is a constant $\kappa^{*}$ depending on the Riemannian metric such that, for every geodesic segment $\alpha$, all sufficiently small $\varepsilon>0$, and all $\delta>0$,

$$
\lim _{T \rightarrow \infty} \lambda_{T, \varepsilon}\left\{\beta:\left|N(\alpha ; \beta)-\kappa^{*}\right| \beta|| \alpha||>\delta T\right\}=0 .
$$

\section{Proof}

This follows from [23, Theorem 7] by the same argument used to prove the ergodic theorem for self-intersections (see [23, Theorem 1]). In the case of constant curvature, $\kappa^{*}=\kappa \Upsilon$, since in constant curvature the Liouville measure and the maximum entropy measure coincide.

Hypothesis 10.1 concerns the quantity $\Delta_{T}^{m} U(x)$ defined by equation (78). For any periodic sequence $x$ with minimum period $\tau(x)=\tau_{T}(x)$ and any integer $m$, this quantity is the maximum difference in self-intersection count between (a) the closed geodesic $G_{x}$ corresponding to the periodic orbit of the suspension flow through $(x, 0)$, and (b) any geodesic segment $G_{y}=\left(\pi \circ \phi_{t}(y, 0)\right)_{0 \leq t \leq S_{\tau(x)} F(y)}$, where $y$ is some sequence that agrees with $x$ in coordinates $-m \leq i \leq \tau(x)+m$. If $m$ is large, any two such geodesic segments are close, because the semiconjugacy between the suspension flow and the geodesic flow is Hölder-continuous. This can be quantified as follows.

\section{LEMMA 11.2}

There exists $A>0$ such that, for all $n \geq 1$, all sufficiently large $m$, and all pairs $x, y \in \Sigma$ such that $x_{i}=y_{i}$ for $-m \leq i \leq n+m$,

$$
d\left(\pi\left(\phi_{t}(x, 0)\right), \pi\left(\phi_{t}(y, 0)\right)\right) \leq e^{-A m} \text { for all } 0 \leq t \leq \tau_{T}(x) .
$$




\section{Proof}

By definition of the "taxicab" metric on $\Sigma_{F}$ (see [13, Definition 2]), the orbits $\phi_{t}(x, 0)$ and $\phi_{t}(y, 0)$ must remain within distance $e^{-B m}$, for a suitable constant $B>0$. Because the semiconjugacy $\pi$ is Hölder-continuous, the projections $\pi \circ \phi_{t}(x, 0)$ and $\pi \circ \phi_{t}(y, 0)$ must remain within distance $e^{-A m}$.

\section{Remark 11.3}

The lengths of the segments $G_{x}$ and $G_{y}$ will in general be different because $S_{\tau(x)} F(y)$ need not equal $S_{\tau(x)} F(x)$. However, the difference in lengths can be at most $\delta_{m}$, where $\delta_{m}$ is given by (84), which decays exponentially in $m$.

\section{Proof of Hypothesis 10.1}

Fix geodesic segments $G_{x}, G_{y}$ as above, and consider the difference in their selfintersection counts. To estimate this, consider how the difference changes as $G_{x}$ is smoothly deformed to $G_{y}$ through geodesic segments by smoothly moving the initial and final endpoints, respectively, along smooth curves $C_{0}$ and $C_{1}$. In such a homotopy, the self-intersection count will change only at intermediate geodesic segments $G_{z}$ along the homotopy where one of the endpoints passes through an interior point of the segment. Now the geodesic segment $G_{z}$ remains within distance $e^{-A m}$ of $G_{x}$, by Lemma 11.2 and Remark 11.3 so for any interior point of $G_{z}$ that meets (say) the initial endpoint of $G_{z}$, the corresponding point on $G_{x}$ must be within distance $e^{-A m}$, and hence within distance $e^{-A m}$ of the curve $C_{0}$. In particular, this corresponding point on $G_{x}$ must fall inside a small rectangle $R_{0}$ surrounding $C_{0}$ whose sides are geodesic arcs. Since the lengths of $C_{0}$ and $C_{1}$ are bounded above by $e^{-A m}+\delta_{m}$ (by Lemma 11.2 and Remark 11.3), the rectangle $R_{0}$ can be chosen so that its sides all have lengths bounded by $e^{-A^{\prime} m}$.

This proves that the difference in the self-intersection counts of $G_{x}$ and $G_{y}$ is bounded above by the number of crossings of $\partial R_{0}$ and $\partial R_{1}$, where $R_{i}$ are rectangles bounded by geodesic arcs of length at most $e^{-A^{\prime} m}$. Hence, Lemma 11.1, for most periodic sequences $x$ of minimal period comparable to $T$ this difference is bounded above by $(8+\varepsilon) e^{-A^{\prime} m} T$ when $T$ is large. This implies Hypothesis 10.1 .

Acknowledgments. The author thanks Moira Chas, Jayadev Athreya, and Dimitri Dolgopyat for some helpful discussions, and he thanks one of the referees for recommending Otal's paper [29].

\section{References}


[2] R. ADLER and L. FLATTO, Geodesic flows, interval maps, and symbolic dynamics, Bull. Amer. Math. Soc. (N.S.) 25 (1991), 229-334. MR 1085823. DOI 10.1090/S0273-0979-1991-16076-3. (1206)

[3] D. V. ANOSOV, Geodesic Flows on Closed Riemann Manifolds with Negative Curvature, Proc. Steklov Inst. Math. 90, Amer. Math. Soc., Providence, 1969. MR 0242194. (1208)

[4] P. BILLINGSLEY, Convergence of Probability Measures, John Wiley, New York, 1968. MR 0233396. (1193, 1203, 1219)

[5] J. S. BIRMAN and C. SERIES, An algorithm for simple curves on surfaces, J. Lond. Math. Soc. (2) 29 (1984), 331-342. MR 0744104. DOI 10.1112/jlms/s2-29.2.331. (1192)

[6] - Geodesics with bounded intersection number on surfaces are sparsely distributed, Topology 24 (1985), 217-225. MR 0793185. DOI 10.1016/0040-9383(85)90056-4. (1192, 1235)

[7] F. BONAHON, Bouts des variétés hyperboliques de dimension 3, Ann. of Math. (2) 124 (1986), 71-158. MR 0847953. DOI 10.2307/1971388. (1228, 1229)

[8] - The geometry of Teichmüller space via geodesic currents, Invent. Math. 92 (1988), 139-162. MR 0931208. DOI 10.1007/BF01393996. (1228)

[9] R. BOWEN, The equidistribution of closed geodesics, Amer. J. Math. 94 (1972), 413-423. MR 0315742. (1194)

[10] - Symbolic dynamics for hyperbolic flows, Amer. J. Math. 95 (1973), 429-460. MR 0339281. (1205, 1206)

[11] Equilibrium States and the Ergodic Theory of Anosov Diffeomorphisms, Lecture Notes in Math. 470, Springer, Berlin, 1975. MR 0442989. (1210, 1214, $1215,1217,1230,1242,1255$ )

[12] R. BOWEN and C. SERIES, Markov maps associated with Fuchsian groups, Publ. Math. Inst. Hautes Études Sci. 50 (1979), 153-170. MR 0556585. (1206, 1207)

[13] R. BOWEN and P. WALTERS, Expansive one-parameter flows, J. Differential Equations 12 (1972), 180-193. MR 0341451. (1205, 1258)

[14] M. CHAS and S. P. LALLEY, Self-intersections in combinatorial topology: Statistical structure, Invent. Math. 188 (2012), 429-463. MR 2909769. (1196)

[15] R. DE LA LLAVE, J. M. MARCO, and R. MORIYÓN, Canonical perturbation theory of Anosov systems and regularity results for the Livšic cohomology equation, Ann. of Math. (2) 123 (1986), 537-611. MR 0840722. DOI 10.2307/1971334. (1208)

[16] M. DENKER and G. KELLER, On U-statistics and v. Mises' statistics for weakly dependent processes, Z. Wahrsch. Verw. Gebiete 64 (1983), 505-522. MR 0717756. DOI 10.1007/BF00534953. (1219)

[17] R. M. DUDLEY, Distances of probability measures and random variables, Ann. Math. Statist. 39 (1968), 1563-1572. MR 0230338. (1250)

[18] M. GROMOV, Three remarks on geodesic dynamics and fundamental group, Enseign. Math. (2) 46 (2000), 391-402. MR 1805410. (1208)

[19] W. HOEFFDING, A class of statistics with asymptotically normal distribution, Ann. Math. Statist. 19 (1948), 293-325. MR 0026294. (1196, 1219) 
[20] S. P. LALLEY, Distribution of periodic orbits of symbolic and Axiom A flows, Adv. in Appl. Math. 8 (1987), 154-193. MR 0886923. DOI 10.1016/0196-8858(87)90012-1. (1194)

[21] Closed geodesics in homology classes on surfaces of variable negative curvature, Duke Math. J. 58 (1989), 795-821. MR 1016446.

DOI 10.1215/S0012-7094-89-05837-7. (1194)

[22] - Renewal theorems in symbolic dynamics, with applications to geodesic flows, non-Euclidean tessellations and their fractal limits, Acta Math. 163 (1989), 1-55. MR 1007619. DOI 10.1007/BF02392732. (1194, 1217, 1246, 1248)

[23] - "Self-intersections of closed geodesics on a negatively curved surface: Statistical regularities" in Convergence in Ergodic Theory and Probability (Columbus, Ohio, 1993), Ohio State Univ. Math. Res. Inst. Publ. 5, de Gruyter, Berlin, 1996, 263-272. MR 1412610. (1192, 1257)

[24] M. LUSTIG, "Paths of geodesics and geometric intersection numbers, II" in Combinatorial Group Theory and Topology (Alta, Utah, 1984), Ann. of Math. Stud. 111, Princeton Univ. Press, Princeton, 1987, 501-543. MR 0895630. (1206)

[25] G. A. MARGULIS, Certain applications of ergodic theory to the investigation of manifolds of negative curvature, Funktsional. Anal. i Prilozhen. 3, no. 4 (1969), 89-90. MR 0257933. (1194, 1246)

[26] - On Some Aspects of the Theory of Anosov Systems, Springer Monogr. Math., Springer, Berlin, 2004. MR 2035655. (1194, 1245)

[27] M. MIRZAKHANI, Growth of the number of simple closed geodesics on hyperbolic surfaces, Ann. of Math. (2) 168 (2008), 97-125. MR 2415399. DOI 10.4007/annals.2008.168.97. (1192)

[28] J. MOSER, On a theorem of Anosov, J. Differential Equations 5 (1969), 411-440. MR 0238357. (1208)

[29] J.-P. OTAL, Le spectre marqué des longueurs des surfaces à courbure négative, Ann. of Math. (2) 131 (1990), 151-162. MR 1038361. DOI 10.2307/1971511. (1229, 1258)

[30] W. PARRY and M. POLLICOTT, An analogue of the prime number theorem for closed orbits of Axiom A flows, Ann. of Math. (2) 118 (1983), 573-591. MR 0727704. DOI 10.2307/2006982. (1206, 1217, 1245, 1246)

[31] Z Zeta Functions and the Periodic Orbit Structure of Hyperbolic Dynamics, Astérisque 187-188, Soc. Math. France, Paris, 1990. MR 1085356. (1210, 1215)

[32] G. P. PATERnAIN, Geodesic Flows, Progr. Math. 180, Birkhäuser, Boston, 1999. MR 1712465. DOI 10.1007/978-1-4612-1600-1. (1205)

[33] M. POLLICOTT and R. SHARP, Angular self-intersections for closed geodesics on surfaces, Proc. Amer. Math. Soc. 134 (2006), 419-426. MR 2176010. DOI 10.1090/S0002-9939-05-08382-6. (1192)

[34] M. RATNER, The central limit theorem for geodesic flows on n-dimensional manifolds of negative curvature, Israel J. Math. 16 (1973), 181-197. MR 0333121. (1193, $1194,1196,1203,1210,1219)$ 
[35] - Markov partitions for Anosov flows on n-dimensional manifolds, Israel J. Math. 15 (1973), 92-114. MR 0339282. (1205)

[36] I. RIVIN, A simpler proof of Mirzakhani's simple curve asymptotics, Geom. Dedicata 114 (2005), 229-235. MR 2174101. DOI 10.1007/s10711-005-7153-1. (1192)

[37] R. SCHOEN and S.-T. YAU, Lectures on Differential Geometry, International Press, Cambridge, Mass., 1994. MR 1333601. (1208)

[38] C. SERIES, Symbolic dynamics for geodesic flows, Acta Math. 146 (1981), 103-128. MR 0594628. DOI 10.1007/BF02392459. (1206, 1207)

[39] D. SIEGMUND, The time until ruin in collective risk theory, Mitt. Verein. Schweiz. Versicherungsmath. 75 (1975), 157-166. MR 0391460. (1219)

[40] V. STRASSEN, The existence of probability measures with given marginals, Ann. Math. Statist. 36 (1965), 423-439. MR 0177430. (1250)

[41] H. WIDOM, Lectures on Integral Equations, Van Nostrand Math. Stud. 17, Van Nostrand, 1969. MR 0243299. (1199)

Department of Statistics, University of Chicago, Chicago, Illinois 60637, USA;

lalley@galton.uchicago.edu 